\title{
AN EXAMINATION OF A CULTURALLY RELEVANT MODEL OF INTUITIVE EATING WITH AFRICAN AMERICAN COLLEGE WOMEN
}

\author{
A Dissertation \\ Presented to \\ The Graduate Faculty of The University of Akron
}

\author{
In Partial Fulfillment \\ of the Requirements for the Degree \\ Doctor of Philosophy
}

Erin C. MacDougall

August, 2010 


\title{
AN EXAMINATION OF A CULTURALLY RELEVANT MODEL OF INTUITIVE EATING WITH AFRICAN AMERICAN COLLEGE WOMEN
}

\author{
Erin C. MacDougall \\ Dissertation
}

Approved:

Advisor

Dr. Linda Subich

Committee Member

Dr. Susan I. Hardin

Committee Member

Dr. Joy Wyatt

Committee Member

Dr. Sandra Perosa

Committee Member

Dr. John Queener
Accepted:

Department Chair

Dr. Paul Levy

Dean of the College

Dr. Chand Midha

Dean of the Graduate School

Dr. George Newkome

Date 


\begin{abstract}
Intuitive eating is a non-diet approach to weight management that encourages people to eat desired food in response to internal signals of hunger and satiety (Tribole \& Resch, 1995; Tylka, 2006). Avalos and Tylka (2006) developed a model of intuitive eating based on the objectification theory (Fredrickson \& Roberts, 1997) and theory of unconditional acceptance (Rogers, 1961; Rogers, 1964). Their model provided an adequate to excellent fit to data obtained from samples of young, mostly European American, college women and explained approximately $43 \%$ of variance in intuitive eating (Avalos \& Tylka, 2006). The present study extended the work of Avalos and Tylka (2006) by exploring the model intuitive eating with a sample of African American college women. In addition, the present study extended the work of Avalos and Tylka (2006) by integrating culturally relevant variables (e.g., racial and ethnic identity) within the model to determine whether the addition of culturally relevant models accounted for additional variance in intuitive eating. Using path analysis procedures with a sample of 130 African American college women, the original model and culturally relevant alternative versions of the model provided an adequate to poor fit to the data. Although models provided an adequate to poor fit to the data, several of the proposed paths were upheld and the models accounted for a sizeable portion of the variance (e.g., approximately $35 \%$ ). Results of the present study provide empirical support for several propositions underlying a model of intuitive eating (Avalos \& Tylka, 2006) and previous
\end{abstract}


research (Augustus-Horvath, 2008; Avalos \& Tylka, 2006) that suggests several, but not all, model paths may extend and generalize to more diverse samples of women. 


\section{TABLE OF CONTENTS}

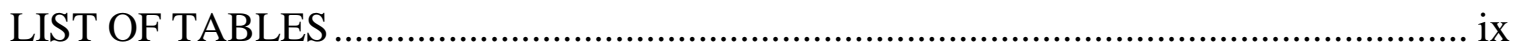

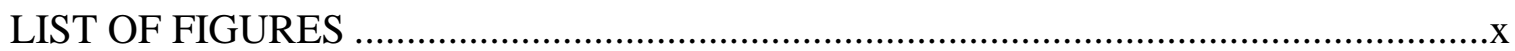

\section{CHAPTER}

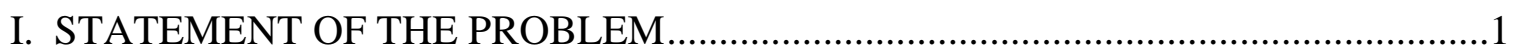

A Positive Approach to Eating Behavior ..............................................................2

African American Women and Eating Behavior ................................................

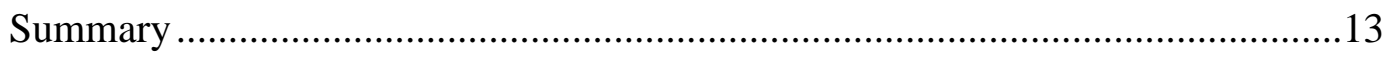

II. REVIEW OF THE LITERATURE ....................................................................15

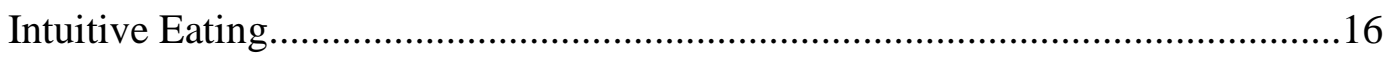

Anti-Dieting Literature as a Foundation for Intuitive Eating …......................17

Principles of Intuitive Eating: An Adaptive Style of Eating.............................20

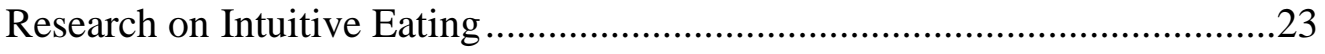

Recent Advances and Limitations in the Area of Intuitive Eating ……...........28

African American Women and Eating Behavior .................................................43

Protective Factors Within the African American Culture..................................45

Identification With African American Culture and Disordered Eating ...........50

Acculturation and Disordered Eating......................................................52 
African Self-Consciousness and Disordered Eating

Racial Identity Attitudes and Disordered Eating ...................................55

Ethnic Identity and Disordered Eating.............................................60



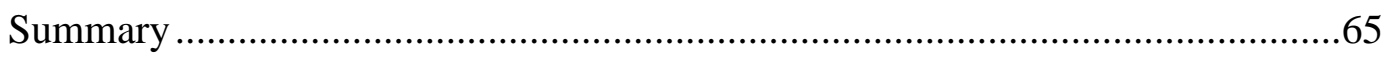

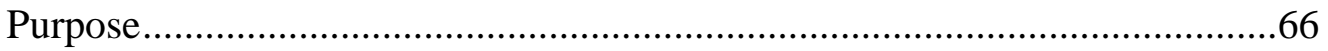

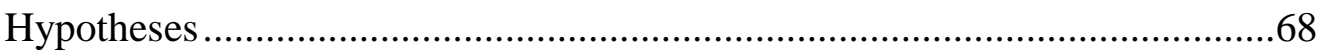

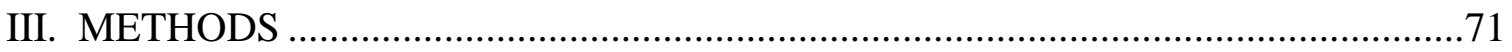

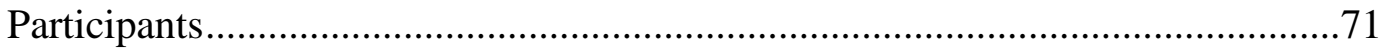

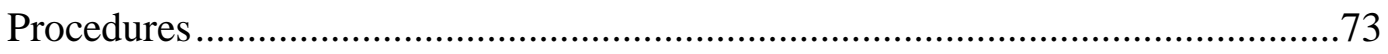

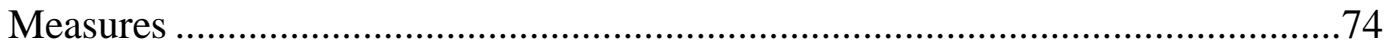

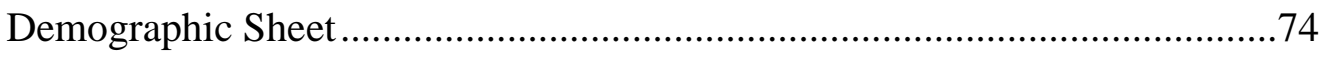

General Unconditional Acceptance From Significant Others .......................74

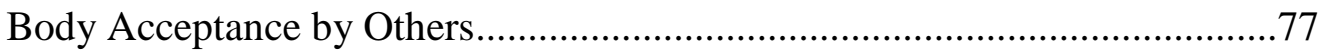



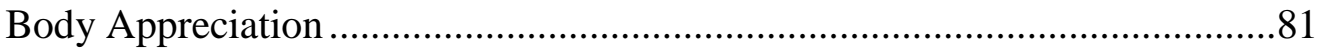

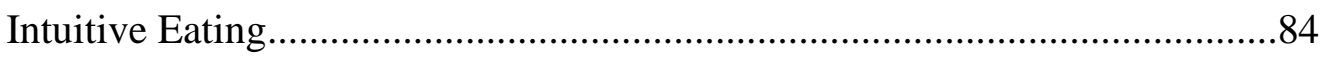

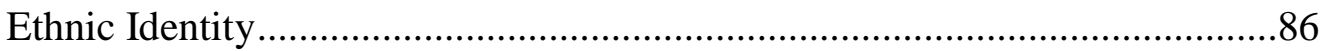

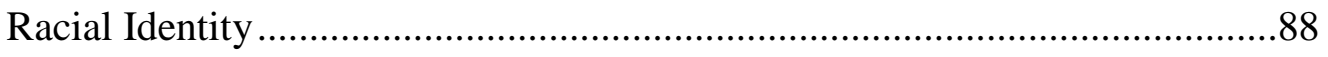

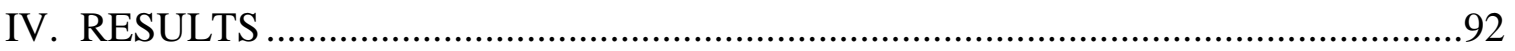

Factor Analysis of Body Appreciation Scale .............................................92

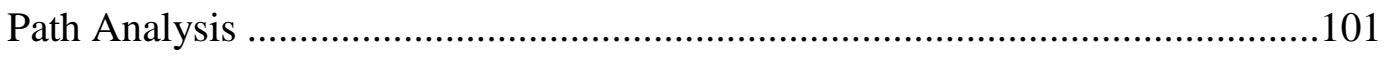

Original Model With Outlier Cases Included in Data Analysis ....................101 
Culturally Relevant Alternative Models With Outlier Cases

Included in Data Analysis

Comparison Among Models

Indirect Effects and Mediation.

Model Modification

Summary

V. DISCUSSION

Comparisons of the Intuitive Eating Models

Specific Relations Among Variables

African American Women's Eating Attitudes 126

Factor Structure of Body Appreciation Scale

Limitations

Implications for Practice and Research.

REFERENCES

APPENDICES

APPENDIX A. DEMOGRAPHIC INFORMATION. 163

APPENDIX B. BARRETT-LENNARD RELATIONSHIP

INVENTORY REVISED

APPENDIX C. BODY ACCEPTANCE BY OTHERS SCALE

APPENDIX D. BODY SURVEILLANCE SUBSCALE OF THE

OBJECTIFIED BODY CONSCIOUSNESS

APPENDIX E. BODY APPRECIATION SCALE

APPENDIX F. INTUITIVE EATING SCALE

APPENDIX G. ETHNIC IDENTITY SUBSCALE OF THE

MULTIGROUP ETHNIC IDENTITY MEASURE .178 
APPENDIX H. INTERNALIZATION AFROCENTRICITY

SUBSCALE OF THE CROSS RACIAL IDENTITY SCALE ........................181

APPENDIX I. RESULTS WITH OUTLIERS EXCLUDED FROM

DATA ANALYSIS

APPENDIX J. TABLES AND FIGURES DEPICTING CONFIRMATORY

FACTOR ANALYSIS OF BODY APPRECIATION SCALE ......................199

APPENDIX K. HUMAN SUBJECTS APPROVAL......................................208 


\section{LIST OF TABLES}

Table $\quad$ Page

1. Means, Standard Deviations, Skewness, and Kurtosis for Measures With Responses of 130 African American College Women ............................................93

2. Correlations Among the Measures for Responses of 130 African American

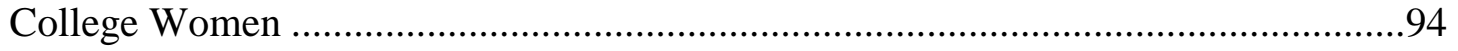

3. Mean, Standard Deviation, and Correlations Among Items on the Body Appreciation Scale With Responses of 130 African American College Women.......96

4. Goodness-of-Fit Indices for Path Analyses for Responses From 130 African American College Women. 


\section{LIST OF FIGURES}

$\begin{array}{lll}\text { Figure } & \text { Page }\end{array}$

1. Original Model of Intuitive Eating Developed and Empirically Examined by

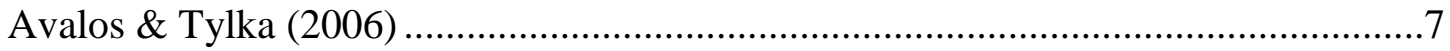

2. Proposed Alternative Model With Culturally Relevant Variables (i.e., Ethnic

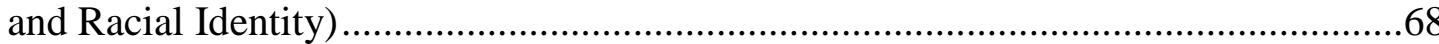

3. Original Model of Intuitive Eating With Path Coefficients Depicted From Data Obtained from 130 African American College Women

4. Culturally Relevant Alternative Model of Intuitive Eating With Racial Identity and Path Coefficients Depicted From Data Obtained From 130 African American College Women

5. Culturally Relevant Alternative Model of Intuitive Eating With Ethnic Identity and Path Coefficients Depicted From Data Obtained From 130 African American College Women. 


\section{CHAPTER I}

\section{STATEMENT OF THE PROBLEM}

A focus on individual strengths and adaptive functioning is one of counseling psychology's unifying characteristics and provided a backdrop for the current investigation. Counseling psychology's dedication to individual strengths and adaptive functioning is apparent in the counseling psychology literature and clinical practice (Gelso \& Fretz, 2001; Harris, Thoresen, \& Lopez, 2007; Watkins, 1983). For example, Lopez, Magyar-Moe, Peterson, Ryder, Krieshok, O’Bryne et al. (2006) analyzed the content of four publications affiliated with counseling psychology (i.e., Journal of Counseling Psychology, The Counseling Psychologist, Journal of Career Assessment, and Journal of Multicultural Counseling and Development) and found that approximately $29 \%$ of the articles included one or more strength-based constructs. The large percentage of counseling psychology articles focusing on strength-based constructs is one indicator of the field's commitment to individual strengths and adaptive functioning. A second indicator of the field's commitment to strengths and adaptive functioning is strengthbased counseling (Smith, 2006). Many counseling psychologists infuse psychotherapy with a focus on strengths by using empirically supported positive constructs (Harris et al., 2007). 
Although counseling psychologists explored and utilized positive constructs throughout the decades in their research and clinical practice, Lopez et al. (2006) implored researchers to "develop and refine theoretical frameworks for positive constructs and processes" (p. 223) and to examine positive constructs with diverse samples. When exploring positive constructs with diverse samples, Constantine and Sue (2006) noted that cultural values, beliefs, and practices are imperative to consider. Thus, exploring models of positive constructs that include a consideration of cultural values, beliefs, and practices with diverse samples contributes to the counseling psychology literature.

\section{A Positive Approach to Eating Behavior}

Although counseling psychology emphasizes individual strengths and adaptive functioning and scientific evidence underscores the importance of considering adaptive along with maladaptive behaviors, the body image and eating behavior research of counseling psychologists focuses almost exclusively on negative components consistent with a pathology-related framework (Avalos, Tylka, \& Wood-Barcalow, 2005; Avalos \& Tylka, 2006; Tylka, 2006; Tylka \& Wilcox, 2006; Wood-Barcalow, 2006). In the counseling psychology literature, numerous correlates and predictors of body dissatisfaction and eating disorder symptomatology have been identified and explored, whereas few correlates or predictors of body satisfaction and adaptive eating have been postulated or empirically examined (Avalos et al., 2005; Avalos \& Tylka, 2006; Molloy \& Herzberger, 1998). 
In addition, correlates and predictors of body dissatisfaction and eating disorder symptomatology have been combined into theoretical frameworks. For example, Frederickson and Roberts (1997) developed the objectification theory to explain how living in a culture that sexually objectifies women leads to disordered eating. According to their objectification theory, interpersonal and societal messages encourage women to lose weight and strive toward a thin-ideal. As women are encouraged to lose weight they internalize the thin-ideal and evaluate themselves according to their physical appearance. Women evaluating themselves against unrealistic societal standards often come up short and experience shame about their body. They may attempt to decrease body shame by ignoring hunger cues and striving to lose weight. Although predictors and models of body dissatisfaction and eating disorders are important, they ignore the strength-based perspective of counseling psychology and adaptive aspects of mental health.

Although much of the body image and eating behavior research focused on negative components, some researchers (e.g., Tylka \& Subich, 1999; Tylka \& Subich, 2004) examined adaptive eating. However, these researchers operationalized adaptive eating as low levels of eating disorder symptomatology (Tylka, 2006). Tribole and Resch (1995) argued that adaptive eating is different than low levels of eating disorder symptomatology. Adaptive eaters rely on physiological signals of hunger and satiety to determine when and how much to eat; moreover, adaptive eaters make food choices without guilt and enjoy the experience of eating (Tribole \& Resch, 1995; Tylka, 2006). To illustrate the differences between low levels of eating disorder symptomatology and adaptive eating, Tylka (2006) described an individual who eats "everything on the plate with no regard to satiety level” (p. 226). Although this person displays low levels of 
eating disorder symptomatology, he/she fails to use internal signals of hunger or satiety to guide eating.

Counseling psychologists (Avalos et al., 2005; Tylka, 2006) subsequently operationalized body appreciation and intuitive eating as aspects of positive body image and eating behavior. And a small body of scientific literature supports body appreciation and intuitive eating as adaptive constructs with White, middle or upper-middle class, college women (Augustus-Horvath, 2008; Avalos et al., 2005; Avalos \& Tylka, 2006; Tylka, 2006; Tylka \& Wilcox, 2006).

According to Avalos et al. (2005), body appreciation is an aspect of positive body image, and it reflects acceptance of the body regardless of physical imperfections or congruence with societal standards of beauty or thinness. Individuals who unconditionally accept their body demonstrate respect for it by engaging in health promoting behaviors (e.g., gentle nutrition) and rejecting the unrealistic standard of beauty or thinness propagated in the media and/or encouraged by family/friends.

Avalos and Tylka (2006) defined intuitive eating as a "strong connection with, understanding of, and eating in response to internal physiological hunger and satiety cues, coupled with a low preoccupation with food" (p. 486). Moreover, authors (Tribole \& Resch, 1995; Tylka, 2006) have identified unconditional permission to eat (when hungry and what food is desired), eating for physical rather than emotional reasons, and reliance on internal hunger and satiety cues to determine when and how much to eat as central features of intuitive eating (Tylka, 2006, p. 226). Individuals who allow themselves unconditional permission to eat do not rely on dietary rules or social cues to determine when, how much, or what foods to eat. Instead, they "honor their hunger" (Tribole \& 
Resch, 1995, p. 17) by eating a sufficient amount of food desired at that moment to quench physical hunger rather than to cope with negative emotions (e.g., boredom, depression, or stress). Individuals who eat intuitively are attuned to their internal experience of hunger and satiety and use these internal experiences to guide eating (Avalos \& Tylka, 2006; Tribole \& Resch, 1995; Tylka, 2006).

Attention to internal experience is central to the process of intuitive eating and is associated with well-being (Tribole \& Resch, 1995; Tylka, 2006). Thus, it was not surprising that when Tylka (2006) explored the association between intuitive eating and other psychological constructs (e.g., well-being, body dissatisfaction, and eating disorder symptomatology), she found that each of the three central features of intuitive eating (i.e., unconditional permission to eat, eating for physical rather than emotional reasons, and reliance on internal signals to determine when and how much food to eat) related moderately and positively to measures of well-being (i.e., self-esteem, life satisfaction, proactive coping, and optimism) and negatively to body dissatisfaction and eating disorder symptomatology.

Tylka and Wilcox (2006) extended the examination of intuitive eating and wellbeing by exploring intuitive eating's incremental validity over low levels of eating disorder symptomatology in predicting well-being. Results of Tylka and Wilcox's exploration suggest that two features of intuitive eating (i.e., eating for physical rather than emotional reasons and reliance on internal hunger and satiety cues) predict wellbeing above and beyond low levels of eating disorder symptomatology.

Based on theoretical writing and extant data, Avalos and Tylka (2006) proposed a model to explain factors that contribute to body appreciation and intuitive eating, and 
they empirically investigated this model with two samples of mostly White, middle class, college women. Specifically, Avalos and Tylka based their model of intuitive eating on objectification theory (Frederickson \& Roberts, 1997) and self-actualization theory (Rogers, 1961, 1964). Because research on objectification theory (e.g., Buchanan, Fischer, Tokar, \& Yoder, 2008; Frederickson \& Roberts, 1997; Moradi \& Rottenstein, 2007; Tylka \& Hill, 2004) supports the notion that negative interpersonal and societal messages contribute to disordered eating through self-objectification and body shame, Avalos and Tylka (2006) postulated that positive interpersonal and societal messages (e.g., unconditional acceptance by others and unconditional body acceptance) could contribute to intuitive eating through a focus on how the body feels and functions over appearance (i.e., body function) and body appreciation.

Path analysis with one sample of young, mostly White, middle class, college women and latent variable structural equation modeling with their other sample revealed that their model provided an adequate fit to the data. In Avalos and Tylka's model of body appreciation and intuitive eating, perceived unconditional acceptance from important others influenced perceived body acceptance by others. Body acceptance by others influenced a focus on how the body feels and functions over appearance. Body acceptance by others and body function influenced body appreciation. Body appreciation and body function influenced intuitive eating. Furthermore, body function and body appreciation mediated the relationship between body acceptance and intuitive eating (see Figure 1). 




Figure 1. Original model of intuitive eating developed and empirically examined by Avalos \& Tylka (2006).

Using structural equation modeling with multiple group analysis, AugustusHorvath (2008) replicated and extended the work of Avalos and Tylka (2006) with women of diverse ages (18-79 years old). The model provided an adequate fit to the data for women of all ages; however, structural invariance suggested that model variables were related at different strengths for younger (i.e., 18-24) and older (i.e., 24-79) women. Overall, then, researchers found support for body appreciation and intuitive eating as aspects of positive body image and healthy eating behavior, and a model of intuitive eating has been developed and examined empirically (Augustus-Horvath, 2008; Avalos \& Tylka, 2006). Most of the participants in these studies, however, were White, middle to upper-middle class women enrolled in psychology courses. Thus, it was important to determine whether these constructs apply to other samples (e.g., women of color, men, children, and athletes). Avalos and Tylka (2006) urged researchers to explore 
their findings with "other samples of women and men, as it is unknown whether the model findings can be extended to fit others' experience and whether intuitive eating is adaptive for more diverse populations" (p. 496).

\section{African American Women and Eating Behavior}

Data from a small number of African American women provide preliminary evidence for the importance of examining body appreciation and intuitive eating (Avalos \& Tylka, 2006; Tylka, 2006). Tylka (2006) collapsed data gathered from four samples of college women participating in the development and psychometric evaluation of a measure of intuitive eating. When data for the Intuitive Eating Scale (IES) was collapsed across all four samples, six racial and ethnic groups emerged (i.e., African American, Asian American, Caucasian American, Latin American, Native American, and Multiracial). It is interesting that a visual comparison of the mean IES scores revealed that African Americans $(n=68)$ had higher total IES scores and IES subscale (Unconditional Permission to Eat, Eating for Physical Reasons, and Reliance on Hunger/ Satiety Cues) scores than did Caucasian American women $(n=1,087)$. In fact, African American women had the highest mean IES total and subscale scores among all the racial and ethnic groups examined.

Furthermore, as a post hoc analysis to their SEM analyses, Avalos and Tylka (2006) examined correlations among their five measures (i.e., Barrett-Lennard Relationship Inventory, Body Acceptance by Others Scale, Body Surveillance subscale of the Objectified Body Consciousness Scale, Body Appreciation Scale, Intuitive Eating Scale) for 38 African American women and 323 European American women 
participating in research on their model of intuitive eating. One relationship significantly differed between the samples. Specifically, the relationship between body function and intuitive eating was stronger for European American than for African American women. The authors concluded that "examination of bivariate correlations between our European and African American participants provides preliminary evidence for the generalizability of some, but not all, variable relationships" (pg. 496).

Although the results of Tylka (2006) and Avalos and Tylka (2006) provide initial support for the generalizability of intuitive eating and body appreciation with samples of African American women, their results should be interpreted with extreme caution. First, the small numbers of African American and large numbers of middle to upper-middle class, European American, college women involved in the research limit results' validity and generalizability. Second, as great heterogeneity exists within the African American community, researchers must be careful to consider individual difference factors such as the identification with African American culture or socioeconomic status of their African American participants.

More specifically, when examining eating behaviors among African Americans, researchers need to consider the impact of culturally specific risk and protective factors (Constantine \& Sue, 2006; Talleyrand, 2002; Wilcox, 2007). Researchers exploring eating disorders and eating disorder symptomatology among African American women already identified racism/ discrimination (Talleyrand, 2002; Wilcox, 2007) and internalization of African American culture and standards of beauty (Abrams, Allen, \& Gray, 1993; Harris, 1994; Harris, 1995) as culturally specific risk and protective factors, 
respectively. These factors are important to examine in conjunction with African American women's body appreciation and intuitive eating.

Talleyrand (2002) explored the relationship between perceived racism, racial identity development, and disordered eating in a sample of African American non-college women. Participants completed the Black Racial Identity Attitudes Scale (Helms, 1990), Schedule of Racist Events (Landrine \& Klonoff, 1996), Compulsive Eating Questionnaire (Golden, Buzcek, \& Robbins, 1986), Emotional Eating Scale (Arnow, Kenardy, \& Agras, 1995), and Restraint Scale (Herman, 1978). Findings suggested that experiences with racism and strong identification with European American culture directly influence eating disorder symptomatology.

If identification with European American culture influences eating disorder symptomatology among African American women, then identification with African American culture and internalization of African American standards of beauty may provide some protection (Striegel-Moore \& Smolak, 1996). Specific factors in the African American community (e.g., reduced emphasis on achieving the thin-ideal, expanded definition of beauty, and presence of supportive relationships) may protect some African American women from body dissatisfaction and disordered eating (Choate, 2005; Constantine \& Sue, 2006; Parker, Nichter, Nichter, \& Vuckovic, 1995).

Reduced emphasis on achieving the thin-ideal in African American culture may relate to the belief that African American men prefer larger women (Choate, 2005). Available research suggests this belief may be accurate. Freedman, Carter, Sbrocco and Gray (2004), for example, explored African American and European American men's preference for women's body size and body shape. African American and European 
American men were recruited from college and community organizations to participate in a study of physical appearance. Participants viewed female stimulus figures representing various body sizes and shapes. Researchers asked participants to identify their ideal and their least favorite female figures. Seventy percent of European American and 30\% of African American men selected the light stimulus figure (body mass index $=15$ ) as ideal; in contrast, 31\% of African American and 6\% of European American men chose the heavy figure (body mass index $=20$ ) as ideal. These results suggest that African American men are more accepting of heavier figures than are their European American counterparts. African American men's preferences, thus, may serve as a protective factor against eating and body image pathology in African American women.

Furthermore, definitions of beauty in the African American community do not rely exclusively on body shape and size; in fact, beauty may encompass many physical (i.e., skin color, hairstyle, and body shape) and psychological factors (i.e., selfconfidence, attitude, and high levels of racial/ethnic identity) (Abrams et al., 1993; Buchanan et al., 2008; Choate,2005; Falconer \& Neville, 2000). When asked to select indicators of beauty, African American women selected more non-weight indicators (e.g., skin color, clothes, and hairstyle) than did their European American counterparts (Harrison \& Stoner, 1976 as cited in Hall, 1995).

Relatedly, research suggested that African American girls and women compliment each other on personal style and appearance instead of encouraging each other to diet and lose weight (Choate, 2005). This presence of supportive relationships, in conjunction with expanded definitions of attractiveness, may protect African American women from developing body dissatisfaction and eating disorders. 
These protective factors may be understood as aspects of African American culture; thus, researchers have examined the assertion that a strong identification with African American culture (i.e., internalization of African American standards of attractiveness) protects African American women from eating disorder symptomatology. Some studies (e.g., Abram et al., 1993; Falconer \& Neville, 2000; Hargreaves et al., 2002; Harris, 1994; Harris, 1995) have supported the premise that a strong identification with the African American community protects African American women from some eating disorder and body image concerns, but other studies have failed to support the claim (e.g., Lester \& Petrie, 1998). Inconsistent terminology and measurement, however, make it difficult to compare findings and reach definitive conclusions.

For example, Abrams et al. (1993) administered self-report measures of disordered eating behaviors (i.e., binge and restraint), disordered eating attitudes (i.e., drive for thinness, body dissatisfaction, and fear of fatness), and assimilation to African American culture to African American women enrolled in college. Results suggested that one eating disorder behavior (i.e., restraint) and two disordered eating attitudes (i.e., fear of fat and drive for thinness) significantly related to the students' level of assimilation to African American culture. Specifically, women less assimilated to African American culture showed higher rates of dietary restraint, fear of fat, and drive for thinness.

Operationalizing the cultural aspect somewhat differently, Harris (1995) examined the relationship between sociocultural factors (i.e., social self-esteem and racial identity development) and body image attitudes in sample of African American college students. Women with high levels of racial identity development, evidenced by a 
positive view of African American culture, held more favorable attitudes toward their physical appearance and endorsed healthier eating and exercise habits.

Taking yet another approach with a sample of African American college women attending historically Black colleges, Falconer and Neville (2000) examined the relation of African self-consciousness and skin color satisfaction to body image. Results indicated an association between African self-consciousness and one dimension of body image satisfaction. Higher levels of African self-consciousness corresponded with higher satisfaction with a specific body area; however, drawing a sample from historically Black college may limit this study's generalizability.

Overall, then, although the operationalization of identification with African American culture has varied, the literature on African American women and eating behavior supports an association between some eating disorder symptomatology, negative body image, and a lower identification with African American culture. Yet, no research has examined the association of body appreciation, adaptive eating, and identification with African American culture. Therefore, exploring a model of intuitive eating that includes a consideration of identification with African American culture, as a protective factor, extends the body image and eating behavior literature on African American women.

\section{Summary}

In addition to extending the body image and eating behavior literature, exploring a model of intuitive eating with a sample of African American women answers calls from numerous authors to extend counseling psychology's focus on human strengths to 
members of diverse groups. Lopez et al. (2006) called researchers to explore positive constructs (e.g., body appreciation and intuitive eating) within a theoretical framework, and Constantine and Sue (2006) urged researchers to consider the importance of cultural values, beliefs, and practices when exploring adaptive functioning in diverse samples. The current project answers these calls. 


\section{CHAPTER II}

\section{REVIEW OF THE LITERATURE}

This study extended the extant literature on intuitive eating by exploring a model of intuitive eating with a sample of African American college women and positing ethnic and racial identity as culturally relevant variables in the model. The present chapter contains two major sections. In the first section, the theoretical and research foundations for intuitive eating are presented. This section begins with a brief review of the antidieting literature, because anti-dieting is a foundation for theoretical writing and empirical research on intuitive eating. Next, the theoretical writings and empirical research on intuitive eating from a variety of academic disciplines are presented. Then, a model of intuitive eating and available research on the model are described. In the second section, the literature on African American women's eating behavior is reviewed and identification with African American culture (e.g., ethnic and racial identity) is offered as a mechanism that may facilitate intuitive eating among African American women. As part of this discussion, studies that explored the association between identification with African American culture and eating behaviors are reviewed. Finally, the chapter concludes with a brief summary and iteration of study objectives and hypotheses. 


\section{Intuitive Eating}

Intuitive eating is defined as a "strong connection with, understanding of, and eating in response to internal physiological hunger and satiety cues" (Avalos \& Tylka, 2006, p. 486). Intuitive eaters recognize the internal signals of hunger and satiety and give themselves permission to eat the amount and type of food desired to quell biological hunger (Tylka, 2006).

Intuitive eating is relatively new to counseling psychology, but other health care professionals (e.g., dieticians, nurses, public health administrators) have explored this construct for some time (Bacon, Kleim, Van Loan, Derricote, Gale, Kazaks, \& Sterns, 2002; Carper, Fisher, \& Birch, 2000; Cole, 2006; Gast \& Hawks, 1998; Polivy \& Herman, 1992; Smith \& Hawks, 2006; Tribole \& Resch, 1995). In 1995, registered dieticians Evelyn Tribole and Elyse Resch used the anti-dieting literature and their clinical experience to identify four styles of eating (i.e., careful eater, professional dieter, unconscious eater, and intuitive eater) and ten principles of intuitive eating. Subsequent health care professionals used Tribole and Resch's ten principles of intuitive eating as the basis for a measure of intuitive eating (Hawks, Merrill, Madanat, Miyagawa, Suwanteerangkul, Guarin, \& Shaofand, 2004), provided empirical support for intuitive eating (Smith \& Hawks, 2006), and developed intuitive eating programs for weight management (Cole, 2006; Leibman, 2005). Until recently, however, intuitive eating was absent from counseling psychology's discussions of eating disorders and adaptive psychological functioning (Tylka, 2006).

In 2006, counseling psychologists (Avalos \& Tylka; 2006; Tylka, 2006; Tylka \& Wilcox, 2006) began to extend intuitive eating research by exploring its correlates and 
predictors. Results of these studies were integrated into literature on eating behaviors and adaptive psychological functioning. As part of their research on correlates and predictors of intuitive eating, counseling psychologists also clarified the definition of intuitive eating and established a psychometrically sound measure of it.

In the following sections, the anti-dieting literature is discussed as a foundation of intuitive eating, the four eating styles and ten principles of intuitive eating are presented (Tribole \& Resch, 1995), research on intuitive eating from professionals within other disciplines is reviewed (Cole, 2006; Hawks et al., 2004; Leibman, 2005; Smith \& Hawks, 2006), and correlates and predictors of intuitive eating are specified (Augustus-Horvath, 2008; Avalos \& Tylka; 2006; Tylka, 2006, 2007; Tylka \& Wilcox, 2006). Limitations and areas for further study are discussed throughout.

\section{Anti-Dieting Literature as a Foundation for Intuitive Eating}

Anti-dieting literature asserts that traditional approaches to weight loss do not work and produce harmful side effects. A number of non-diet approaches to weight management emerged to address the oversights of traditional weight loss strategies (Cole, 2006). The following discussion is not a comprehensive review of traditional or nontraditional approaches to weight management; rather, it is a brief introduction to the anti-dieting literature, and it is intended to provide a foundation for Tribole and Resch's (1995) proposal of four styles of eating and ten principles of intuitive eating.

Weight loss strategies traditionally fall into one of three categories (Gast \& Hawks, 1998; Mann, Tomiyama, Westling, Lew, Samuels, \& Chatman, 2007; Powell, Calvin, \& Calvin, 2007). Categories include food restriction, medical intervention, and 
lifestyle change. Food restriction is the reduction or elimination of certain foods (e.g., ice cream) or food groups (e.g., carbohydrates) in hopes of losing weight. Research suggests that food restriction works for short but not long-term weight loss. Mann et al. (2007) reviewed 14 longitudinal studies, in which participants were asked to record their weight before participating in a restrictive diet, immediately after the diet, and at specific followup points for at least 4 years. On average, participants reported a 31-pound weight loss immediately following the restrictive diet, but most participants gained back all but six pounds at final follow-up. In addition, approximately $40 \%$ of the participants weighed more at final follow-up than at baseline. Mann et al. (2007) concluded that food restriction does not produce long-term weight loss.

Anti-dieting literature proclaims that food restriction diets do not work and produce harmful side effects including a preoccupation with body size and shape, slow metabolism, binge eating, and weight cycling (Carrier, Steinhardt, \& Bowman, 1994; Steinhardt, Bezner, \& Adams, 1999; Tribole \& Resch, 1995). In addition, diets teach people to distrust themselves and their internal signals of hunger and satiety. Instead of relying on internal signals, diets encourage people to rely on external rules that govern type, time, and amount of food consumed (Tribole \& Resch, 1995).

Medical intervention is the second broad category of weight loss strategies and includes the use of drug therapy (e.g., orlistat, sibutramine) or weight loss surgery (e.g., gastric bypass). Medical interventions achieve modest, but clinically significant, changes in weight, but these modest changes in weight come at some level of risk. Individuals using drug therapy for weight loss may experience negative side effects (e.g., neurotoxicity and hypertension) and become reliant on drugs for weight management. 
Moreover, the discontinuation of some drug therapies is associated with weight gain. Risks associated with weight loss surgery are significant and life threatening (Cole, 2006; Powell et al., 2007).

Lifestyle change is the third category of weight loss and includes dietary change, behavior modification, and exercise. Powell et al. (2007) reviewed seven randomized clinical trials that examined the long-term effects of lifestyle changes. They concluded that lifestyle changes produce modest reduction in weight (i.e., 7 lbs.) and reduce risk for diabetes and hypertension. Most successful lifestyle changes included dietary modification and exercise.

It is clear from the research that some traditional weight loss strategies produce modest weight loss and reduce risk for serious health conditions; however, none of the traditional approaches to weight loss address the genetic, environmental, or psychological correlates of body size or shape. In fact, traditional weight loss programs assume people can revise their bodies and achieve the thin-ideal perpetuated in Western culture, regardless of physiology or psychological variables (Cole, 2006).

In the past 15 years, a number of non-diet approaches to weight management emerged to address the oversights of traditional weight loss strategies (Cole, 2006). Instead of focusing on weight loss, non-diet approaches encourage individuals to accept a range of body shapes and sizes, to recognize physiological hunger and satiety, and to eat in response to physiological hunger (Bacon et al., 2002; Bacon, Van Loan, Stern \& Keim, 2005; Carrier et al., 1993; Cole, 2006; Savoye, Berry, Dziura, Shaw, Serrecchia, \& 2002; Steinhardt et al., 1999; Tribole \& Resch, 1995). Eat for L.I.F.E, Diet Free Forever, Bright Bodies Weight Management Program, and Health at Every Size are four examples 
of nontraditional weight loss programs (Bacon et al., 2002; Bacon et al., 2005; Carrier et al., 1993; Cole, 2006; Savoye et al., 2002; Steinhardt et al., 1999; Tribole \& Resch, 1995).

Research supports the benefits of Eat for L.I.F.E, Diet Free Forever, Bright Bodies Weight Management Program, and Health at Every Size. Participants in these anti-dieting programs improved on measures of self-esteem and body acceptance (Bacon et al., 2002; Bacon et al., 2005; Carrier et al., 1993; Steinhardt et al., 1999). In addition, participants reduced dietary restraints and depression (Carrier et al., 1993; Steinhardt et al., 1999). Furthermore, allowing individuals to eat according to physical or emotional hunger had no adverse consequences on biomedical variables; in fact, a few research studies suggest that participants in anti-dieting programs reduced total cholesterol and blood pressure (Bacon et al., 2002; Bacon et al., 2005; Cole, 2006). In addition, individuals in anti-dieting programs showed higher rates of program completion and were more likely to maintain improvements at follow-up (Bacon et al., 2002; Bacon et al., 2005). Such anti-dieting programs appear healthy for mind and body.

\section{Principles of Intuitive Eating: An Adaptive Style of Eating}

Intuitive eating is a non-diet approach to weight management that discourages individuals from using dietary rules or food restriction for weight control; instead, intuitive eating encourages individuals to eat desired food in response to internal signals of hunger and satiety (Augustus-Horvath, 2008; Avalos \& Tylka, 2006; Tribole \& Resch, 1995; Tylka, 2006; Tylka \& Wilcox, 2006; Tylka, 2007). By eliminating dietary regulations and restoring a connection with internal signals (i.e., hunger and satiety), 
intuitive eating, like other anti-dieting programs, is healthy for both mind and body (Tylka, 2006).

Tribole and Resch (1995) described four styles of eating (i.e., the careful eater, professional dieter, unconscious eater, and intuitive eater) to help individuals clarify their eating style. Categorizing individuals' eating style and exploring consequences of their dietary pattern are important steps in learning how to become an intuitive eater. The careful eater is someone who examines and anguishes over every piece of food consumed. Because careful eaters spend large amounts of time laboring over ingredients and methods of preparation, they often undereat. Similar to the careful eater, the professional dieter spends large amounts of time carefully planning meals and snacks. Specifically, professional dieters spend time analyzing the nutritional content of their food and ensuring portion control. Unlike the careful eater, the professional dieter occasionally goes off the diet and eats large portions of forbidden food. Periodic binges prevent the professional dieter from long-term weight loss. An unconscious eater is someone who pairs eating with another task or activity (e.g., watching television). Because eating is often ignored, the unconscious eater frequently overeats and gains weight. Finally, intuitive eating is a style of eating that relies on internal hunger and satiety cues to determine when and how much to eat.

In addition to these four styles of eating, Tribole and Resch (1995) described ten principles of intuitive eating. Rejecting the false promise of dieting is the first principle of intuitive eating. In lieu of food and energy deprivation, these authors proposed via their second principle to keep the body physiologically fed. Feeding the body with energy rich foods (i.e., carbohydrates) fends off the primal drive to overeat when 
starving. Articulated in principle three is that granting oneself permission to eat when hungry the food desired at that moment prevents intense craving and binges. Permission to eat requires challenging strict dietary rules (e.g., I should not eat after $6 \mathrm{pm}$ ) and restriction, and the authors assert this as their fourth principle. Principle five states that individuals need to monitor their fullness and stop eating when satiated. In order to enjoy the food eaten, principle six suggests taking time to savor food in a comfortable environment. Eating when hungry and stopping when full suggests using food to meet physical instead of emotional needs. According to principle seven, intuitive eaters do not use food to cope with negative emotional states such as boredom, anxiety, or loneliness. In addition to respecting internal hunger and satiety cues, intuitive eaters also respect their genetic blueprints. Principle eight describes intuitive eaters as rejecting unrealistic expectations for their body size or shape propagated in the media and/or encouraged by family/friends. Finally, principles nine and ten suggest caring for the body by exercising and practicing gentle nutrition. Gentle nutrition uses taste as one-factor in food choice, but also considers health and nutrition.

Tribole and Resch (1995) clearly described clients' difficulty losing weight with traditional diets and their four styles of eating and ten principles of intuitive eating in Intuitive Eating: A Recovery Book for the Chronic Dieter: Rediscover the Pleasures of Eating and Rebuild Your Body Image. They did not describe, however, the clients who inspired their work on intuitive eating. Describing the clients who inspired the work would have grounded their proposals in clinical data. Tribole and Resch also did not speculate on the application of intuitive eating to diverse populations (e.g., men or racial 
minorities), or propose relationships among principles. Finally, they offered no empirical support for their principles.

\section{Research on Intuitive Eating}

Initially, little empirical evidence existed to support the construct of intuitive eating or the principles associated with it. Indeed, it was suggested that, "empirical research is needed to determine if intuitive eaters have healthier diets than the general population and if the assumption of the emergence of a naturally thin body is accurate. Empirical evidence is also needed to determine if weight loss will be maintained or at least stabilized by applying” (Gast \& Hawks, 2000, p. 43) principles of intuitive eating. Recognizing this lack of empirical support, health care professionals investigated the relationship between intuitive eating, nutrition, and weight management. In addition, they developed and evaluated weight management programs based on principles of intuitive eating (Cole, 2006; Gast \& Hawks, 2000; Hawks, Madanat, Hawks, \& Harris, 2005; Smith \& Hawks, 2006).

To aid in the investigation of intuitive eating, Hawks, Merrill, and Madanat (2004) developed a 27-item self-report Intuitive Eating Scale. Their Intuitive Eating Scale has four subscales that represent principles of intuitive eating. Specifically, the measure includes intrinsic eating, extrinsic eating, anti-dieting, and self-care subscales. Intrinsic eating assesses the tendency to eat according to internal signals of hunger and satiety. Extrinsic eating assesses the tendency to eat according to external factors such as social situations, environmental cues, or emotional states. Anti-dieting represents aversion to food restriction. Finally, self-care assesses one's emphasis on body function 
over appearance. Reverse coding items from the extrinsic eating scale and then averaging four subscale scores determines the Intuitive Eating Scale total score. Higher total scores indicate greater adherence to principles of intuitive eating. The authors reported that initial alpha coefficients and test/retest reliability were adequate for each subscale; however, they failed to report actual values.

Hawks, Merrill, Mandanat, Miyagawa, Suwanteerangkul, Guarin, and Shaofand (2004) used the intuitive eating scale to evaluate agreement with principles of intuitive eating among a sample of college students from the United States and Asia. Specifically, they administered the Intuitive Eating Scale to 2,334 undergraduate students in Japan, Thailand, China, the Philippines, and the United States. The structure of the data obtained from Asian participants was compared to that obtained from participants in the United States. The authors did not report the specific factor extraction method; however, they noted that four factors (i.e., intrinsic eating, extrinsic eating, anti-dieting, and selfcare) emerged with U.S. and Asian participants. Although four factors emerged, individual subscale items needed some adjustments. The authors deleted items with factor loadings less than .4 on their intended scale "to make subscales more culturally relevant to each country" (Hawks, Merrill, Mandanat, Miyagawa, Suwanteerangkul, Guarin, \& Shaofand, 2004, p.197). Making subscales culturally relevant required the authors to delete three items for Japan, five items for Philippines, and six for China. The majority of deleted items came from the anti-dieting and intrinsic eating subscales.

Once subscales were adjusted for cultural relevance, the authors (Hawks, Merrill, Mandanat, Miyagawa, Suwanteerangkul, Guarin, \& Shaofand, 2004) examined differences among countries with intuitive eating subscale and total scores. An 
unspecified test of group differences indicated that: the participants from the United States and Japan scored lower on intrinsic eating subscales than participants from other countries; the participants from the United States scored highest; and participants from Thailand scored lowest on the extrinsic eating and self-care subscales. Although results provided some evidence of structural validity and cross-cultural differences in intuitive eating, they need to be interpreted with extreme caution. The authors did not report specific statistical analyses or values generated from statistical analyses. In addition, directly comparing culturally relevant subscales with varying numbers of items may have elicited inaccurate results.

Hawks et al. (2005) used the Intuitive Eating Scale, nevertheless, to examine the relationship between intuitive eating and various health indictors (i.e., body mass index, triglyceride level, levels of high-density lipoproteins, and cardiovascular health) among a sample of female college students. Intuitive eating related negatively to body mass index and triglyceride level. In addition, intuitive eating related positively to high-density lipoproteins and cardiovascular health. Results supported the assumption that high levels of intuitive eating promote health among a small, healthy, sample of college women at a single university; yet, the cross-sectional design of this study prevented examination of causal relationships.

Subsequently, Smith and Hawks (2006) evaluated the relationship between intuitive eating, diet composition, and meaning associated with food. Specifically, the authors examined the relationship between scores on the Intuitive Eating Scale and diet composition, body mass index, health consciousness, and pleasure associated with eating in a homogeneous sample of healthy college students. Results of statistical analysis 
suggested that higher scores on the Intuitive Eating Scale were associated with a lower body mass index, less anxiety about eating, and fewer dieting behaviors. In addition, higher scores on the Intuitive Eating Scale were associated with greater enjoyment of eating. Although the authors concluded that intuitive eating is associated with many health-promoting behaviors, instruments contained in this survey limit the validity and reliability of the results. Specifically, the authors created or modified many of the surveys used in this study; moreover, some of these surveys contained a limited number of items (e.g., three surveys used two or fewer items to assess complex constructs).

Despite the problems noted with research on intuitive eating, some health care professionals and eating disorder centers (i.e., Center for Change) promote intuitive eating as an effective weight management and eating disorder treatment (Smith \& Hawks, 2006). To assess the veracity of these claims, Cole (2006) examined the effectiveness of intuitive eating as a weight management program.

Cole (2006) developed, implemented, and evaluated a weight management program based on the principles of intuitive eating. She collaborated with potential program participants and developed "My Body Knows When," a non-diet approach to weight management based upon the principles of intuitive eating. By surveying and directly interacting with potential program participants, Cole determined potential program participants' readiness for lifestyle change and developed appropriate intervention strategies. Over the course of ten sessions, participants discussed negative physical and psychological consequences of dieting, fear of gaining weight without diets, coping with emotions without food, disease prevention, and the importance of regular physical activity. To increase participant involvement in weekly sessions and program 
retention, Cole generated discussion questions, in-session activities, and homework assignments. For example, the Barriers to Activity Quiz was developed for a session focused on exercise; this quiz helped participants identify and overcome barriers to physical activity (e.g., limited time).

To evaluate program effectiveness, Cole randomly assigned 87 participants to a treatment or control group. Prior to the start of the program six treatment group participants and 20 control group participants quit; therefore, the program actually began with 38 treatment group participants and 23 control group participants. An additional 15 treatment group participants and 12 control group participants quit either during the program or immediately after the program; thus, 18 treatment group participants and 14 control group participants remained at the end of the study. The small numbers of participants at the end of the study, due to the $53 \%$ attrition rate, reduced the statistical power and limited the results of the study.

Participants completed surveys created for the study before they began the nondiet program, immediately after the program, and at a 6-month follow-up. Surveys used in pre-, post- and follow-up testing assessed nutrition, quality of life and aspirations, dietary and non-dietary behaviors, intuitive eating, readiness for change, and barriers to intuitive eating. In addition to completing the self-report surveys, anthropometric measurements (i.e., body mass index, waist circumference, hip circumference, and bicep circumference) and a 3-day diet record were obtained from participants. Cole (2006) noted that anthropometric measurements were taken from participants wearing winter clothes and including winter apparel in body measurement may have imparted some measurement error. 
Results suggested that program participants learned principles of intuitive eating and incorporated two intuitive eating principles (i.e., rejecting a diet mentality and identifying feelings of hunger and satiety) into their long-term weight management plan. Specifically, $95 \%$ of program participants reported giving up restrictive dieting altogether. Results should be interpreted with extreme caution, however, because of the high attrition rate, small sample size, self-report nature of dietary and non-dietary measures, use of unvalidated surveys, and possible anthropometric measurement error (Cole, 2006).

In sum, research evidence offers some support for the association of intuitive eating with various health indicators (e.g., body mass index, blood lipid profiles, and cardiovascular health) as well as its utility as part of an effective weight management program. Establishing such associations between intuitive eating and positive outcomes is important, but additional research is needed to address correlates and predictors of intuitive eating. Indeed, Hawks et al. (2005) suggested that future research should "address the questions of causality, and... evaluate the health efficacy of intuitive eating among diverse populations" (p. 335).

\section{Recent Advances and Limitations in the Area of Intuitive Eating}

Counseling psychologists extended the study of intuitive eating by exploring its predictors and correlates and integrating intuitive eating into the eating behavior and adaptive functioning literature (e.g., Augustus-Horvath, 2008; Avalos \& Tylka; 2006; Tylka, 2006, 2007; Tylka \& Wilcox, 2006). In order to establish a firm foundation for research, Tylka (2006) defined intuitive eating as eating in response to physiological 
hunger and satiety cues. In addition, she described three central and interrelated features of intuitive eating: unconditional permission to eat when hungry and what food is desired, eating for physical rather than emotional reasons, and reliance on internal hunger and satiety cues to determine when and how much to eat (Tylka, 2006, p. 226). Individuals who eat intuitively recognize the internal signals of hunger (e.g., gurgling in the stomach, irritability, headache, and fatigue) and eat the type and amount of desired food to subdue internal signals of hunger without guilt (Tribole \& Resch, 1995; Tylka, 2006).

Defining intuitive eating as an adaptive behavior with three central and interrelated features distinguishes it from measures of body image or body appreciation (Tylka, 2006). Body image is a construct that includes a range of attitudes, although most studies explore negative body image (e.g., body image disturbance) (WoodBarcalow, 2005). Body appreciation is an acceptance and respect for the body regardless of imperfections or congruence with the thin-ideal (Avalos et al., 2005). It is expected that individuals with positive body image or body appreciation respect their bodies by engaging in health promoting behaviors (e.g., intuitive eating) (Avalos \& Tylka, 2006).

With this clear definition of intuitive eating, Tylka (2006) developed and psychometrically evaluated an Intuitive Eating Scale (IES). She collected data in four studies from approximately 1,200 participants. Tylka developed items for the IES using extant theoretical and empirical writings on intuitive eating (e.g., Tribole \& Resch, 1995), although she failed to mention whether she used the Intuitive Eating Scale developed and empirically evaluated by Hawks, Merrill, and Madanat (2004). In study one, Tylka explored the factor structure of the IES and determined whether IES scores were valid and reliable among a sample of 391 young, mostly White, college women. In addition, 
Tylka explored the scale's construct validity by administering similar and related measures (i.e., EAT-26 [Garner, Olmsted, Bohr, \& Garfinkel, 1982], Body Dissatisfaction and Introspective Awareness subscales of EDI-2 [Garner, 1991]; Perceived Sociocultural Pressure scale [Stice, Ziemba, Margolis, \& Flick, 1996]; Internalization subscale of Sociocultural Attitudes Toward Appearance [Heinberg, Thompson, \& Stormer, 1995]). Exploratory factor analysis determined that a three-factor solution, which corresponded to central features of intuitive eating, fit the data best; moreover, internal consistency reliabilities for scores on IES were strong. Correlations between the IES and related measures were as expected; specifically, scores on IES related in a negative direction to eating disorder symptomatology, poor introspective awareness, pressure for thinness from significant others, and internalization of the thinideal stereotype.

In the second study, Tylka (2006) conducted a confirmatory factor analysis with data obtained from a second sample of 476 young, mostly White, women enrolled in psychology courses at a Midwestern university to determine whether items loaded on their hypothesized factor and whether there were relationships between the expected three factors and a higher level construct. In addition, Tylka explored the scale's construct validity by again administering similar (i.e., Rosenberg Self-Esteem Scale [Rosenberg, 1965]; Life Orientation Test-Revised [Scheier, Carver, \& Bridges, 1994]; Proactive Coping subscale of Proactive Coping Inventory [Greenglass, Schwarzer, \& Taubert, 1999]; Satisfaction with Life Scale [Diener, Emmons, Larsen, \& Griffin, 1985]) and dissimilar measures (i.e., Impression Management subscale of the Balanced Inventory of Desired Responding [Paulhus,1994]). Confirmatory factor analysis revealed 
that the three factors related moderately to one another and loaded on a broader intuitive eating construct. Correlations between the IES and the additional measures were as expected. Specifically, scores on IES related in a positive direction to self-esteem, satisfaction with life, optimism, and proactive coping; scores on the IES did not relate to impression management.

Tylka (2006) examined the relationship between body mass index and intuitive eating in study three with a sample of 199 young, mostly White, women enrolled in introductory psychology courses. She found an association between intuitive eating and body mass index; specifically, individuals who honored their internal signals of hunger and satiety were more likely to weigh an appropriate amount for their height.

Correlations between the IES and body mass index, calculated from participants' selfreported weight and height, supported previous research (Hawks et al., 2005) and provided additional construct validity for the IES.

Finally, in study four, Tylka (2006) explored the scale's test-retest reliability by giving 194 young, mostly White, college women enrolled in psychology classes at both a regional campus and main campus of a large Midwest university the IES, and then readministering the IES 3 weeks later. Results suggested that total IES and subscale scores were relatively stable over 3 weeks.

As the samples used in development and evaluation of IES were mostly White college women, it is imperative to explore its psychometric properties with men and with women of color (Tylka, 2006). Also, surprisingly, many of the items on the IES assessed negative components consistent with a pathology-related framework, and then were reverse scored (Tylka, 2006). Recall, intuitive eating is not the simple the lack of 
maladaptive eating but rather the presence of an adaptive style of eating. Items that assess presence of maladaptive eating behaviors and then are reverse scored may not capture the full essence of intuitive eating (Avalos \& Tylka, 2006; Tylka, 2006; Swami \& Chamorro-Premuzic, 2008).

Although most participants in Tylka's development and psychometric evaluation of IES were White, middle class, college women, a small number of ethnic minority women participated. The data from the small number of ethnic minority participants allowed for a preliminary evaluation of cultural differences. Six ethnic groups (African American, Asian American, Caucasian, Latin American, Native American, and Multiracial) emerged when information was collapsed across the four intuitive eating scale studies. As an appendix to her article, Tylka (2006) reported means and standard deviations for her total sample of women and for each of the six ethnic groups.

An inspection of the mean IES scores revealed that African Americans had higher total IES and IES subscale scores than did the Caucasian Americans. In fact, African American women had the highest mean IES total and subscale scores among all ethnic groups, and Caucasian women had the lowest mean IES total and unconditional permission to eat subscale scores among all ethnic groups. These IES score differences may suggest the presence of protective factors or risk factors inherent in women's group membership. Specifically, higher levels of intuitive eating among African American women may suggest the presence of protective factors (e.g., greater acceptance of a diversity of body shapes, expanded definition of beauty, and presence of supportive peers). These results, however, should be interpreted with caution because of the small number of ethnic minority women $(\mathrm{n}=172)$ and the large numbers of European 
American women $(\mathrm{n}=1,087)$. Additional research is needed to empirically explore racial/ethnic differences in intuitive eating and determine the presence of protective or risk factors inherent in racial/ethnic group membership.

Tylka (2006) reported that intuitive eating scores were related in a positive direction to measures of well-being (i.e., satisfaction with life, optimism, proactive coping) and in a negative direction to eating disorder symptomatology. Tylka and Wilcox (2006) extended this line of inquiry and explored whether intuitive eating and eating disorder symptomatology are low and high levels of the same construct. In their first study, Tylka and Wilcox (2006) explored whether intuitive eating predicted variance in positive affect, self-esteem, and proactive coping beyond that accounted for by low levels of eating disorder symptomatology. Research assistants administered the Positive Affect subscale of the Positive and Negative Affect Schedule Expanded (Watson, Clark, \& Tellegan, 1988), Rosenberg Self-Esteem Scale (Rosenberg, 1965), the Proactive Coping subscale of the Proactive Coping Inventory (Greenglass et al., 1999), the Intuitive Eating Scale (Tylka, 2006), and the EAT-26 (Garner et al., 1982) to a sample of mostly White, college women enrolled in an introduction to psychology course at a large, Midwestern university. The authors used hierarchical multiple regression and determined that intuitive eating contributed to the three well-being indexes (i.e., positive affect, selfesteem, and proactive coping) after controlling for eating disorder symptomatology. As expected, eating for physical rather than emotional reasons and reliance on internal hunger and satiety cues to determine when and how much to eat made unique contributions to well-being. Unconditional permission to eat when hungry what food is desired overlapped with eating disorder symptomatology. 
In their second study, Tylka and Wilcox (2006) explored whether intuitive eating predicted four additional measures of well-being (i.e., optimism, unconditional selfregard, psychological hardiness, and social problem solving) beyond contributions made by eating disorder symptomatology. Research assistants administered the Life Orientation Test-Revised (Scheier, Carver, \& Bridges, 1994), Unconditional Self-Regard Scale (Betz, Wohlgemuth, Serling, Harshbarger, \& Klein, 1995), Psychological Hardiness Scale (Betz \& Campbell, 2003), Social Problems Solving Inventory (D’Zurilla, Nezu, \& Maydeu-Olivares, 1997), Intuitive Eating Scale (Tylka, 2006), and the EAT-26 (Garner et al., 1982) to 397 women enrolled in psychology courses. Again the authors used hierarchical multiple regression and determined that intuitive eating made unique contributions to optimism, unconditional self-regard, psychological hardiness, and social problem solving after controlling for contributions from eating disorder symptomatology. Eating for physical rather than emotional reasons and reliance on internal hunger and satiety cues to determine when and how much to eat made unique contributions to these adaptive psychological resources. Again, unconditional permission to eat when hungry what food is desired overlapped with eating disorder symptomatology.

From these two studies, the authors concluded that intuitive eating and eating disorder symptomatology are not different levels of the same construct. In terms of specific components of intuitive eating, however, only eating for physical rather than emotional reasons and reliance on hunger and satiety cues were described as distinct from low levels of eating disorder symptomatology. These results are limited, however, by the exclusive use of self-report methodology. Tylka and Wilcox (2006) note that the assessments of intuitive eating and well-being relied on participants' accurate report of 
current level of functioning, and individuals with low levels of intuitive eating may not be attuned to their internal physiological or psychological experiences.

Although intuitive eating was identified as an adaptive eating behavior and correlates of intuitive eating had been established, no predictors or model integrating predictors had yet been offered (Tylka, 2006; Tylka \& Wilcox, 2006). Recognizing this gap in the literature, Avalos and Tylka (2006) identified predictors of adaptive eating, postulated a model of body appreciation and intuitive eating, empirically investigated this model with a sample of mostly White, middle class, college women, and cross-validated the model with another sample of mostly White, middle class, college women.

The authors argued that intuitive eating is an innate quality but that certain environmental influences disrupt this eating style (Avalos \& Tylka, 2006). Numerous authors (Buchanan et al., 2008; Moradi, Dirks, \& Matteson,2005; Moradi \& Rottenstein, 2007; Tylka \& Hill, 2004) had established pathways between negative environmental influences (e.g., objectification) and eating disorders, but Avalos and Tylka (2006) argued that positive environmental conditions (e.g., acceptance) could contribute to a positive body image and adaptive eating behaviors.

Their hypothesized model of intuitive eating was based on both Carl Rogers' theory of unconditional acceptance and included five variables (i.e., general unconditional acceptance, body acceptance by others, body function, body appreciation, and intuitive eating). General unconditional acceptance is a belief that important others accept a woman for her real self and do not want her to abandon her "true qualities" (Avalos \& Tylka, 2006, p. 487). Body acceptance by others is a belief that others accept a woman's body and do not wish her to modify external appearance by dieting. Body 
function is an emphasis on internal experiences over external appearance. Body appreciation reflects approval and respect for one's body regardless of imperfections or congruence with societal standards of beauty or thinness. A woman with body appreciation cares for her body by engaging in healthy behaviors and rejecting the unrealistic body images portrayed in the media. Individuals who focus on internal experiences rather than outward appearance and appreciate their body in spite of imperfections respect their body by engaging in principles of intuitive eating (Avalos \& Tylka, 2006; Tribole \& Resch, 1995).

Avalos and Tylka (2006) asserted the following paths in their hypothesized model of intuitive eating. First, general acceptance from important others was suggested to contribute to both body acceptance by others and body function. Second, body acceptance by others was suggested to contribute directly to body function and body appreciation. Third, body function was suggested to contribute directly to body appreciation and intuitive eating. Fourth, body appreciation was suggested to contribute directly to intuitive eating. Finally, body function and body appreciation were suggested to fully mediate the relationship between acceptance and intuitive eating.

In their research, Avalos and Tylka (2006) obtained the responses of 181 women enrolled in introductory psychology courses to five measures (i.e., Perceived Unconditional Acceptance from Most Influential Other [Barrett-Lennard, 1962; Claiborn, Crawford, \& Hackman, 1983], a modified version of Perceived Sociocultural Pressure Scale [Stice et al., 1996], the Body Surveillance subscale of the Objectified Body Consciousness Scale [McKinley \& Hyde, 1996], the Body Appreciation Scale [Avalos et al., 2005], and the Intuitive Eating Scale [Tylka, 2006]). Participants identified as 
European American (82.2\%), African American (5.0\%), Asian American (3.9\%), and Native American (0.6\%). Exactly $89 \%$ of the participants described themselves as middle or upper middle class. Although participants ranged in age from 17 to 55 years old, their mean age was 20.2 years.

Results of a path analysis procedure suggested that the model provided an excellent fit to the data. In fact, all paths in the model were significant, except the path from general unconditional acceptance to body function. The nonsignficant path was deleted and the truncated model (see Figure 1) was cross-validated with a second sample of college women.

Four hundred and sixteen mostly White, college women were recruited to participate in the cross-validation study of Avalos and Tylka's (2006) model of intuitive eating. Participants in the cross-validation study identified as European American (77.6\%), African American (9.1\%), Asian American (5.0\%), and Latina (2.4\%). More than $89 \%$ of the participants described themselves as middle or upper middle class. The authors used the same measures to cross-validate their prior results, but latent structural equation modeling (SEM) replaced path analysis. In order to provide multiple indicators of latent factors, the authors created parcels from the items of the measures of general unconditional acceptance, body acceptance by others, body function, body appreciation, and intuitive eating. Results suggested that the measurement and structural model provided an adequate fit to the data. In addition, an examination of indirect effects found that body function and body appreciation fully mediated the relationship between body acceptance and intuitive eating. 
Avalos and Tylka's (2006) model explained approximately $42.5 \%$ of the variance in intuitive eating. With more than $57 \%$ of variance in intuitive eating unexplained, Avalos and Tylka (2006) encouraged future researchers "to integrate other interpersonal and intrapersonal variables within the acceptance model" (p. 495).

It should be noted that Avalos and Tylka assessed only the level of perceived acceptance from the most influential person when growing up. Individuals may receive inconsistent messages about themselves and their bodies from the most influential person and others in their environment (Avalos \& Tylka, 2006). More broadly assessing unconditional acceptance by others may provide a better measure of the acceptance, especially among diverse samples.

The percentage of African Americans in the second sample was large enough to conduct post hoc analyses, so Avalos and Tylka (2006) examined intercorrelations among measures for the sample of African American ( $\mathrm{n}=38)$ and European American participants $(n=323)$. Authors used a series of fisher's z test and determined that one pair of bivariate correlations was significantly different between samples; specifically, the correlation between body function and intuitive eating was stronger for European American than for African American women. Overall, these results provided very preliminary evidence for the generalizability of intuitive eating and body appreciation with African American women, however, they must be interpreted with extreme caution (Avalos \& Tylka, 2006).

As Avalos and Tylka (2006) explored their model of intuitive eating with two samples of young, mostly White, college women, additional research is needed to test how well their model fits with diverse samples (i.e., older women, women of color, and 
men). Recognizing the need to explore the model of intuitive eating with diverse samples, Augustus-Horvath (2008) tested Avalos and Tylka's model of intuitive eating with a sample of younger (i.e., 18-24) and older (i.e., 25-79) women.

Augustus-Horvath (2008) solicited 688 women to participate in a study of eating behavior and body image concern from introductory to psychology courses at a Midwestern university and through electronic messages. Participants completed an online survey, which included a demographic questionnaire and measures of unconditional acceptance, body acceptance, body function, body appreciation, and intuitive eating. Augustus-Horvath deviated from Avalos and Tylka's methodology by not using the Barrett-Lennard Relationship Inventory (Barrett-Lennard, 1962; Claiborn et al., 1983) to assess unconditional acceptance. Instead, Augustus-Horvath used the Social Provision Scale (Russell \& Cutrona, 1984; Cutrona \& Russell, 1987) to more broadly measure general unconditional acceptance.

For the research, Augustus-Horvath (2008) divided the sample of 688 women into two age groups (i.e., younger and older). Three hundred and seven mostly White, college women, who ranged in age from 18 to 24 years $(M=19.28 ; S D=1.61)$ comprised the younger group. They identified as European American (80.1\%), Latina (6.2\%), African American (2.9\%), and Asian American (0.3\%). More than 79\% of the participants in the younger group described themselves as upper middle class and $12 \%$ described themselves as middle class. For women in the younger group, body mass indices ranged from 16.10 to $54.8(M=25.64)$.

Three hundred and eighty one mostly White, college women, who ranged in age from 24 to 79 years old $(M=41.43 ; S D=11.39)$ comprised the older group. Of the older 
group, $87.4 \%$ identified as European American, $4.7 \%$ identified as African American, $1.8 \%$ identified as Latina/Hispanic, and 1.6\% identified as Asian American. Negligible percentages of women identified as multiracial, biracial, Native American, international, and/or chose not to specify a race or ethnicity. Eleven percent of the women in the older group identified as upper class and $79.8 \%$ described themselves as middle class. In terms of body mass index, women in the older group weighed significantly more $(\mathrm{p}<$ $.05)$ than women in the younger group. For women in the older group, body mass indices range from 17.8 to $54.8(M=27.1)$.

The author also found significant group differences in perceived social support, body acceptance by others, body function, and intuitive eating. Women in the older group reported higher levels of perceived social support and body function, whereas women in the younger group reported higher levels of body acceptance by others and intuitive eating.

Given the significant difference in body mass index with regard to age group, Augustus-Horvath (2008) decided to include body mass index as a covariate in analysis. With body mass index controlled as a covariate, Augustus-Horvath used structural equation modeling with multiple group analysis to examine the fit of Avalos and Tylka's (2006) model of intuitive eating with data obtained from samples of younger and older women; moreover, the author determined whether model pathways significantly differed between age groups. As expected, the model provided an adequate fit to the data from both age groups. Although the model provided adequate fit for both groups, results suggested model invariance. Across groups, the constructs (i.e., unconditional acceptance, body acceptance, body function, body appreciation, and intuitive eating) 
were associated at different strengths. Results also suggested that the model accounts for a sizeable amount of variance in intuitive eating for both groups; specifically, the model explained approximately $40.1 \%$ of the variance in intuitive eating among the women aged 18-24 and roughly $46.7 \%$ of the variance in intuitive eating among the women aged $24-79$.

Although the results replicated those of Avalos and Tylka (2006) and extended the research on intuitive eating with a sample of older women, they should be interpreted with caution because of sampling methods. First, women attending a large Midwest university were invited to participate in an online study of body image and eating behaviors and interested parties were provided with the URL address of the survey. Internet data collection may inadvertently exclude a subset of women from participating in this study (e.g., women with limited financial resources and computer skills) and generate a sample unrepresentative of the general population (Augustus-Horvath, 2008).

These studies of the Avalos and Tylka model of intuitive eating (AugustusHorvath, 2008; Avalos \& Tylka, 2006) indicate that the model did not account for a majority of the variance in intuitive eating. Tylka (2007), however, explored the roles of two additional intrapersonal variables in an abbreviated model of intuitive eating; specifically, she explored whether self-esteem and proactive coping moderated the relationship between body acceptance by others and intuitive eating with a sample of mostly White college women. Participants completed the Body Acceptance from Others Scale (Avalos et al., 2005), the Rosenberg Self-Esteem Scale (Rosenberg, 1965), the Proactive Coping subscale of the Proactive Coping Inventory (Greenglass et al., 1999), and the Intuitive Eating Scale (Tylka, 2006). Tylka used hierarchical moderated 
regression and determined that self-esteem and proactive coping strengthened the relationship between body acceptance by others and intuitive eating. In addition, selfesteem and proactive coping accounted for $3.3 \%$ and $2.5 \%$ of variance in intuitive eating, respectively. The results of this investigation suggested that other intrapersonal variables could influence the model of intuitive eating and should be considered in future research studies (Tylka, 2007).

Clearly, counseling psychologists advanced the intuitive eating literature by exploring correlates and predictors of intuitive eating; however, results of the extant research are limited to White, middle to upper middle class, college women. Future research needs to explore the construct of intuitive eating with other samples (e.g., community samples, men, women of color). Furthermore, even European American college women are not a homogenous population, and within group differences on background contextual variables (e.g., age, social class), personal characteristics (e.g., self-esteem), and sociocultural factors (e.g., internalization of thin-ideal) merit consideration. In addition, each of the studies presented collected data at a single point in time, so no causal claims can be made about the variables. Longitudinal data are needed to determine whether the model of intuitive eating accurately represents the causal relations in the development of adaptive eating among college women. Finally, intuitive eating has been assessed exclusively via self-report and not through observations of actual eating behaviors. Assessments of intuitive eating could be confounded with an individual's perceptions of eating behaviors that may or may not be accurate (Avalos \& Tylka, 2006; Tylka, 2006; Swami \& Chamorro-Premuzic, 2008). 


\section{African American Women and Eating Behavior}

In the past, researchers considered men and African American women immune from eating disorders and body dissatisfaction (O’Neill, 2003; Wilcox, 2007; Wildes, Emery, \& Simons, 2001); however, recent research with African American women discredited the notion that ethnic minorities are impervious to eating disorders and body dissatisfaction (Striegel-Moore \& Smolak, 2000). In fact, some studies suggest that African American community members (Wilfley, Schreiber, Pike, Striegel-Moore, Wright, \& Rodin, 1996) and college women (Mulholland \& Mintz, 2001) report levels of eating disorder symptomatology similar to European Americans. Other studies, however, find both similarities and differences in prevalence and severity of eating disorder symptomatology among African American and European American participants.

For example, Abrams et al. (1993) found that African American college women reported equivalent levels of purging (i.e., self-induced vomiting and laxative use) and lower levels of dietary restraint and binging than did their European American counterparts. In another study, Cashel, Cunningham, Landeros, Cokley, and Muhammad (2003) recruited African American and European American students from undergraduate psychology courses, sororities, and fraternities. Using this diverse group of college students, authors found equivalent levels of bulimic symptomatology among European American and African American participants and lower levels of dieting, internalization of thin ideal, drive for thinness, and body dissatisfaction among African American participants than reported by their European American counterparts.

Given these similarities and differences in prevalence and severity of eating disorders, researchers (O’Neill, 2003; Wildes et al., 2001) conducted meta-analyses to 
examine the role of ethnicity in eating disorder symptomatology. Wildes et al. (2001) conducted a meta-analysis of 35 studies that examined the role of ethnic group membership in the development of body dissatisfaction and eating disorder symptomatology. More than 17,700 White and non-White (e.g., African American, Arabian, Asian, Hispanic, and Russian) women and men were included in the metaanalysis. Researchers coded outcome measures into eight categories: bulimia nervosa (diagnosed by DSM-IV criteria), eating disorder (diagnosed by researcher), weight and dieting concerns, drive for thinness, dietary restraint, body dissatisfaction, smaller ideal body, and lower reported weight. Because of the lack of research studies comparing anorexia nervosa among White and non-White populations, researchers excluded it from their meta-analysis.

Wildes et al. (2001) calculated the effect size of each outcome measure separately and collapsed outcome measures into an eating disturbance/body dissatisfaction composite measure. When comparing level of eating disturbance and body dissatisfaction for White and African Americans, the effect sizes for all outcome measures were statistically significant and ranged in magnitude from small to large. In fact, the mean effect size of two outcome measures (i.e., dietary restraint and ideal body size) were very large and suggested that African Americans weigh more, report fewer concerns about their weight, and attempt to lose weight through dietary restriction less often than do Whites.

O'Neill (2003) extended the work of the Wildes et al. (2001) by separating men's scores from women's scores. Using data from nearly 26,300 African American and White women, O'Neill examined the relationship between ethnic identity and eating 
disturbance. A variety of eating disturbances (e.g., anorexia, bulimia, binge-eating disorder, and disordered-eating NOS) were examined. As the author hypothesized, the mean effect for ethnicity was significant yet small in magnitude. The small, yet significant, effect suggested that African American women report fewer eating disturbances than do White women; however, the type of eating disturbance was important to consider. Specifically, African American women had significantly lower levels of anorexia and disordered-eating NOS, but not statistically significant lower levels of bulimia or binge-eating disorder. These findings suggest the rate of eating disturbances in African Americans is slightly less than and different from that of European American women, but they do not indicate the absence of eating disturbances among African American women.

\section{Protective Factors Within the African American Culture}

Certain factors in the African American community may protect some African American women from eating disorder symptomatology (Striegel-Moore \& Smolak, 1996). Authors (Choate, 2005; Harris, 1994; Molloy \& Herzberger, 1998) have asserted that African American culture's reduced emphasis on achieving the thin-ideal, greater acceptance of a diversity of body sizes and shapes, expanded definition of beauty, and presence of supportive relationships promote a positive body image and protect some African American women from disordered eating.

Specifically, African American men and women view overweight and obese individuals differently than do European Americans (e.g., Abrams et al., 1993; Akan \& Grilo, 1995; Befort, Thomas, Daley, Rhode, \& Ahluwalia, 2008; Molloy \& Herzberger, 
1998). Befort et al. (2008) conducted a qualitative examination of perceptions and beliefs about body size and shape among 62 obese African American women. African American women queried for this qualitative study expressed acceptance for a variety of body sizes and shapes and believed that African American women could be attractive at any size.

In addition, research indicates that heavier African American women are viewed more positively (Wade \& DiMaria, 2003) than are thinner African American women, whereas thinner European American women are viewed more positively (Wade \& DiMaria, 2003) and as more socially desirable (Wade, Loyden, Renninger, \& Tobey, 2003) than are heavier European American women. In these studies, the researchers presented college students with a picture and description of either a thin or a heavy person of either African American or European American descent; moreover, the researchers asked participants to rate the stimulus person on a variety of dimensions (e.g., attractiveness, intelligence, success). A MANOVA revealed a significant interaction of race and weight such that representations of thin European American women received higher life success, attractiveness, social desirability, and personality ratings than did heavy European American women. Moreover, heavy African American women received higher life success, attractiveness, and personality ratings than did thin African American women. Although results are consistent with the view that African Americans can be attractive at any size (Befort et al., 2008), they should be viewed within the limitations of the study. Specifically, participants were young, mostly White, college students attending a predominantly White university. 
Greater acceptance for a variety of body sizes and shapes among African American women may be related to perception of men's preferences of larger women (Choate, 2005; Molloy \& Herzberger, 1998). Research (Freedman et al., 2004; Greenberg \& LaPorte, 1996) on body type preference suggests that this belief may be accurate. For example, Greenberg and LaPorte (1996) examined differences in body size preferences among African American and European American men. Ninety-eight men recruited from undergraduate courses and 81 men recruited from a diverse community sample ranked, in order of attractiveness, nine racially neutral female figure drawings of varying sizes. In addition, participants responded to statements (e.g., I wish my girlfriend was thinner/heavier) regarding their current romantic relationship. Results suggested that European American men chose significantly thinner figures as attractive and more frequently reported hoping that their girlfriends would lose weight; however, the strength of relations between race and ratings was small and other variables may be important to explore.

In another study, Freedman et al. (2004) examined differences in body size and shape preferences among African American and European American men recruited from college and community organizations. One hundred and three men viewed female figure drawings of varying shapes and sizes. Afterward, participants identified their most and least favorite female figure drawing. Differences emerged in men's preference for women's body shape and size. Results suggested that African American men preferred women with a lower waist-to-hip ratio and who were heavier than did European American men. Nearly $70 \%$ of European American and 30\% of African American men chose the smallest female figure drawing, which represented a woman with a body mass 
index of 15, as their favorite female figure. In contrast, $31 \%$ of African Americans and 6\% of European Americans chose the heaviest female figure drawing, which represented a woman with a body mass index of 20 , as their favorite female figure.

Results of these studies suggest that African American men, relative to their European American counterparts, prefer women with a larger body size (Freedman et al., 2004; Greenberg \& LaPorte, 1996). Furthermore, research suggests that African American men may be more receptive to non-weight indicators of beauty (e.g., clothes, make-up, and skin color) and assess multiple factors when selecting a potential mate as compared to White men (Vaughan, Stewart, \& Sacco, 2004).

Indeed, both African American men and women more often select non-weight indicators of beauty (e.g., clothes, make-up, and skin color) than do their European American counterparts (Falconer \& Neville, 2000; Vaughan et al., 2004). Authors suggest that beauty in the African American community is composed of multiple physical (e.g., skin color, personal style, fit of clothes, hairstyle, and body shape) and non-physical (e.g., self-confidence) indicators. In fact, African American girls and women in this research spoke about looking good and working with what you have (Allan, Mayo, \& Michel, 1993; Falconer \& Neville, 2000; Parker et al., 1995) regardless of congruence with the thin-ideal. Allan et al. (1993) noted that looking good and working with what you have involves personal style, attitude, grooming, fit of clothes, and hairstyle. Therefore, the concept of attractiveness in the African American community may be related more closely to self-confidence and personal style than obtaining the thin-ideal portrayed in the media. 
Relatedly, some literature suggests that African American girls and women recognize physical and non-physical indicators of beauty and complement one another on their appearance (Choate, 2005). African American women report receiving more support to develop a personal style and less pressure to achieve the thin-ideal from peers and significant others as compared to reports by European American women (Lovejoy, 2001). Indeed, in a qualitative study, Befort et al. (2008) asked African American women about body acceptance by others and social support for weight loss. Approximately $50 \%$ of the women queried reported that their family accepted their body regardless of size and discouraged them from losing weight. One participant stated, 'I've never felt judged and probably because most of the women in my family are overweight... I think their acceptance of me for all that I am and not just for how much I weigh has always been just sort of something I could count on” (p. 419).

In another qualitative study, Allan et al. (1993) examined body size values of European American and African American women. Specifically, researchers elicited family values about body size, family and peer reaction to weight loss or gain, and sources of information about body size. Respondents (36 European and 31 African Americans) most frequently mentioned evaluation of one's body size by close friends and family as a factor influencing value of body size. It is interesting that evaluation by others had different salience for European and African American women. European American women perceived partners and family valuing thinness and others wanting them to be thin and lose weight; whereas, African American women perceived others as encouraging them to maintain a healthy body size and not lose weight. In addition, African American women reported that body size and weight were rarely discussed in 
their households. Although not formally discussed, African American women believed the men in their lives "thought they looked just fine" (p. 331) and preferred them to be healthy rather than thin. This presence of supportive peer and community relationships in conjunction with expanded definitions of attractiveness may protect African American women from developing body dissatisfaction and eating disorders.

\section{Identification With African American Culture and Disordered Eating}

Clearly, researchers have identified a number of factors in African American culture that could protect African American women from negative body image and eating disorders (Allan et al., 1993; Befort et al., 2008; Choate, 2005; Falconer \& Neville, 2000; Lovejoy, 2001; Molloy \& Herzberger, 1998). Extending this line of research, a few studies examined the association between identification with African American culture and body dissatisfaction/eating disorder symptomatology (e.g., Abrams et al., 1993; Akan \& Grilo,1995; Aruguete, Nickleberry, \& Yates, 2004; Baugh, 2005; Cachelin \& Regan, 2006; Edwards-Hewitt \& Gray, 1993; Falconer \& Neville, 2000; Harris, 1994; Pumariega, 1986; Spadafore, 2008). Some of these studies support an association between identification with African American culture and body dissatisfaction/eating disturbance (Abram et al., 1993; Cachelin \& Regan, 2006; Harris, 1994; Falconer \& Neville, 2000; Peterson, Rojhani, Steinhaus, \& Larkin, 2000; Pumariega, 1986; Sabik, Cole, \& Ward, 2007; Spadafore, 2008) whereas others fail to support such an association (Aruguete et al., 2004; Spadafore, 2008).

Given this lack of consensus in findings, Wildes et al. (2001) examined the relationship between identification with African American culture and eating disturbance 
as part of the meta-analysis mentioned previously. Across three studies, the mean effect size was small and nonsignificant $(d=.23)$. Too few studies reported on the relationship between African American culture and eating disturbance to draw definitive conclusion.

An alternative explanation for the lack of consensus among researchers examining the relationship between identification with African American culture and eating disturbance is the presence of methodological inconsistencies. In this body of literature, differences in research design make direct comparisons across studies difficult and definitive conclusions impossible to reach (Wildes et al., 2001). For example, researchers use a variety of terms and instruments to operationally define identification with African American culture (e.g., ethnic identity, racial identity, acculturation, assimilation, African racial consciousness) and body dissatisfaction/eating disturbance (e.g., bulimia, eating disorder, weight and dieting concern, dietary restraint, drive for thinness, body dissatisfaction, smaller ideal body, lower reported weight, purging). To complicate the situation, some researchers use terms interchangeably. For example, one study explored the relationship between "acculturation/assimilation" (Akan \& Grilo, 1995, p. 183) within African American and Asian American individuals. Although the authors used acculturation and assimilation interchangeably, these terms have very different meanings. Acculturation refers to the process of acquiring cultural norms, values, and standards of dominant culture, whereas assimilation refers to an interactive process where both majority and minority culture influence one another (Atkinson, Morten, \& Sue, 1998). In an attempt to minimize confusion in the extant literature, this brief review discusses studies of acculturation, African self-consciousness, racial identity, and ethnic identity, separately. 
Acculturation and disordered eating. Acculturation refers to changes within an individual or culture after contact with a more dominant culture (Atkinson et al., 1998). Contact with European American culture transforms African American cultural norms, values, and standards of beauty (Osvold \& Sodowsky, 1993). Researchers have hypothesized that African American women who acculturate to European American culture are at a greater risk for eating disorder symptomatology than are African American women who maintain African American norms, values, and standards of beauty (Aruguete et al., 2004; Osvold \& Sodowsky, 1993). Researchers have used a variety of acculturation questionnaires and measures to assess acculturation to both European American (Pumariega, 1986) and African American cultures (Aruguete et al., 2004; Spadafore, 2008).

Pumariega (1986) examined the association between acculturation to "AngloAmerican" culture, eating disorder symptomatology, and socioeconomic status among non-White adolescent girls. One hundred and thirty eight adolescent girls completed the Eating Attitudes Test (Garner et al., 1982), Acculturation Questionnaire (Pumariega, 1986), and the Hollingshead-Redlich Two-Factor Index of Social Position (Hollingshead, 1957). The Acculturation Questionnaire assessed a range of cultural variables (e.g., years in the United States, language, food, music, and friends). Scores on the Acculturation Questionnaire indicated most adolescents were highly acculturated to "Anglo-American" culture. In addition, results indicated that acculturation to "Anglo-American" culture was associated with eating behavior. Specifically, a higher level of acculturation to the dominant culture was associated with higher scores on the Eating Attitudes Test. The results of this study support the association between acculturation and eating 
disturbances; however, the acculturation measure used in this study limits results. The author devised the Acculturation Questionnaire specifically for this study and failed to report any psychometric information.

Spadafore (2008) provided additional evidence for the association between acculturation and eating disturbance. The author measured acculturation to African American culture and eating disorder symptomatology (e.g., binging, purging, and dietary restriction) in a sample of African American women attending a historically Black college. Results suggested a negative association between acculturation to African American culture and some eating disorder symptomatology; specifically, higher levels of acculturation to African American culture were associated with lower levels of purging. Results, however, suggested no association between acculturation and binging or dietary restraint. The results of this correlational study suggest that acculturation to African American culture is associated with some, but not all, eating disorder symptomatology among African American women attending a historically Black college. Although Pumariega (1986) and Spadafore (2008) found an association between acculturation and some eating disorder symptomatology, another study (Aruguete et al., 2004) failed to find such a relationship. Aruguete et al. (2004) examined the constructs of "Black acculturation," body image, and eating attitudes among 50 Black and 26 White students attending a historically Black college. Male and female participants completed the Eating Attitudes Test (Garner et al., 1982), the Body Mass Silhouette Scale (Canadian Dietetic Association, 1988), the Body Esteem Scale (Mendelson, Mendelson, \& White, 2001), and the Acculturation Questionnaire of African Acculturation Scale (Landrine \& Klonoff, 1994). As the authors expected, Black participants reported higher levels of 
African American Acculturation and fewer disturbed eating attitudes and behaviors than their White counterparts reported. In addition, Black participants selected larger figures on the Body Mass Silhouette Scale to represent their current and ideal body shape. Contrary to expectations, acculturation was not associated with eating attitudes or body image among Black or White participants; however, authors note that results are limited by the small sample size.

In conclusion, some research (Pumariega, 1986; Spadafore, 2008) suggests a significant association between acculturation and eating disorder symptomatology whereas other research (Aruguete et al., 2004) fails to support the association. Lack of consistent findings may have been due, however, to small effect sizes, low power, or slight variations in research methodology (e.g., sample size, sample type, terminology, and instruments).

African self-consciousness and disordered eating. African self-consciousness refers to “African Americans' self-awareness of and practices regarding their historical, cultural, linguistic, and philosophical origins as African-descended people" (Falconer \& Neville, 2000, p. 237) and typically is measured by the African Self-Consciousness scale (Baldwin \& Bell, 1982, 1985). Higher levels of African self-consciousness are expected to protect African American women from body dissatisfaction and eating disorder symptomatology (Falconer \& Neville, 2000).

Falconer and Neville (2000) used the African Self-Consciousness Scale (Baldwin \& Bell, 1982, 1985) to examine the association between African American culture and body image perceptions among 124 African American women attending a historically 
Black university. In addition to the African Self-Consciousness Scale, participants completed the Skin Color Satisfaction Scale, a questionnaire developed specially for this study, and three subscales of the Multidimensional Body-Self Relations Questionnaire (Cash, Winstead, \& Janda, 1986).

As the authors' hypothesized, cultural variables were associated with body image satisfaction. Specifically, African self-consciousness was associated with women's satisfaction with discrete body features (e.g., hair, torso, weight, height) and overall appearance. These findings suggest that cultural variables are related to African American women's body-perceptions; however, results are based on a small sample of African American women attending a historically Black university. In addition,the authors created the Skin Color Satisfaction Scale especially for this study and provided limited data to support the psychometric properties of the scale.

Racial identity attitudes and disordered eating. The Racial Identity Attitude Scale (RIAS; Parham \& Helms, 1981) and The Racial Identity Attitude Scale for Blacks (RIAS-B; Helms, 1990) assess an individual's identification with both African American and European American cultures; however, authors (e.g., Fischer, Tokar, \& Serna, 1998; Lemon \& Waehler, 1996; Tokar \& Fischer, 1998) have criticized the RIAS and RIAS-B for low internal consistency and inconsistent structural validity. Despite publicized concerns about the psychometric properties of the RIAS and RIAS-B, numerous authors have used these scales to operationalize racial identity attitudes (Worrell \& Watson, 2008). 
The RIAS and RIAS-B identify four stages of racial consciousness: preencounter, encounter, immersion-emersion, and internalization (Helms, 1990; Parham \& Helms, 1981). Individuals in the preencounter stage idealize White culture and denigrate African American culture. Individuals in the encounter stage are in the process of discovering African American culture and questioning mainstream White culture. After a period of questioning mainstream cultural norms and values, individuals in the immersionemersion stage engross themselves in African American culture and loathe majority White culture. Finally, individuals in the internalization stage appreciate African heritage and recognize the presence of other salient identities (Cross \& Vandiver, 2001; Parham \& Helms, 1981; Worrell, Cross, \& Vandiver, 2001). It would be expected that African American women with preencounter attitudes have the highest level of body dissatisfaction (Makkar \& Strube, 1995) and eating disturbance (Dinsmore \& Mallinckrodt, 2001).

Using the RIAS-B, Abrams et al. (1993) explored the association between racial identity and disordered eating attitudes and behaviors among a sample of 95 young, African American, college students. Disordered eating behaviors were measured using the Binge Scale (Hawkins \& Clement, 1980) and the Restraint Scale (Herman \& Mack, 1975). Disordered eating attitudes were measured using the Drive for Thinness and Body Dissatisfaction subscales of the Eating Disorder Inventory (Garner, Olmsted, \& Polivy, 1983), Diagnostic Survey of Eating Disorders (Johnson, 1984), and Goldfarb Fear of Fat Scale (Goldfarb, Dykens, \& Gerrard, 1985). Assimilation to White culture, reflected by the preencounter stage of RIAS, was positively related to dietary restraint, drive for thinness, and fear of fat. Results, however, failed to support a relationship between racial 
identity attitudes and binge eating or body dissatisfaction. The small, yet significant, correlations between dietary restraint, drive for thinness, and fear of fat and the preencounter stage of RIAS-B suggest that ascription to White cultural standards may put African American women at risk for restrictive eating disorders.

In related research, Harris (1995) explored the relationship between personal, family, and societal characteristics and body image attitudes among a small sample of African American college women. Harris used simple correlations and multiple regression analysis to examine the relationship between personal, family, and societal characteristics and body image. Results suggest a significant association between racial identity attitudes and body image attitudes such that individuals in more advanced levels of racial identity development (i.e., encounter, immersion, and internalization) reported greater satisfaction with their physical appearance. Racial identity, however, failed to predict satisfaction with physical appearance when other personal, family, and societal characteristics were included in the analysis.

Although some studies suggest that levels of racial identity attitudes are associated with eating disorder symptomatology and body dissatisfaction, other studies (Akan \& Grilo, 1995; Edwards-Hewitt \& Gray, 1993) indicate no association. For example, Edwards-Hewitt and Gray (1993) administered the RIAS to 138 African American college students from three universities (i.e., a private, predominantly Black college, a private, predominantly White college, a public, predominantly Black college) and examined the association between racial identity attitudes and eating disorders. In order to identify individuals with clinical eating disorders, Edwards-Hewitt and Grey administered the Eating Attitudes Test (Garner et al., 1982), Drive for Thinness and Body 
Dissatisfaction subscales of the Eating Disorder Inventory (Garner et al., 1983), and the Restraint Scale (Herman, 1987). Only a few African American participants reported symptoms of anorexia and/or subclinical eating disorders; therefore, Edwards-Hewitt and Gray discarded these persons from subsequent analyses. Nine African American participants reported a set of symptoms consistent with a diagnosis of bulimia nervosa. Of these women, eight were categorized in the internalization stage of racial identity development, according to scores on RIAS. Although most of the women meeting diagnostic criteria for bulimia nervosa were in the internalization stage of racial identity development, statistical analysis failed to support a significant relationship between racial identity development and bulimia nervosa.

Akan and Grilo (1995) also found no relationship between racial identity attitudes and eating disorder symptomatology or body dissatisfaction. Akan and Grilo examined the relationship between acculturation in Asian Americans and assimilation in African Americans and "eating attitudes/behaviors" (p. 183). Researchers used the Eating Attitudes Test (Garner \& Garfinkel, 1979), Goldfarb Fear of Fat Scale (Goldfarb et al., 1985), Eating Disorder Examination-Questionnaire (Fairburn \& Wilson, 1993), Physical Appearance Related Testing Scale (Thompson, Fabian, Moulton, Dunn, \& Altabe, 1991), and the Body Shape Questionnaire (Cooper, Taylor, Cooper, \& Fairburn, 1987) to measure "eating attitudes/ behaviors". Researchers used the RIAS-B-short form (Helms, 1990) and Suinn-Lew Acculturation Scale (Suinn, Ahuna, \& Khoo, 1992) to measure assimilation and acculturation, respectively.

In order to examine the relationship between assimilation/acculturation and eating attitudes/behavior among African American participants, Akan and Grilo (1995) grouped 
participants into three categories (i.e., preencounter, immersion, and internalization) based on RIAS-B scores. The authors noted that African American participants whose scores fell in the bottom one third of all scores were placed in the preencounter category, which reflects an idealization of mainstream culture (including standards of beauty). Individuals whose scores fell in the middle third of all scores were placed into the immersion category, which reflects a rejection of mainstream culture dominated by European American standards. Finally, individuals whose scores fell in the top one third of all scores were placed into the internalization category that reflects an appreciation of African American and European American culture. The authors noted no relationship between assimilation/acculturation and eating attitudes/behaviors, but they failed to provide data or results of statistical analysis for readers' consideration.

In sum, a number of studies support the association between internalization of racial attitudes and restrictive eating disorders and body dissatisfaction (Abrams et al., 1993; Harris, 1995). The results of these studies appear consistent with claims that internalization of African American culture and other cultures (ex: White culture) might place African American women at risk for eating disturbances and body dissatisfaction (Dinsmore \& Mallinckrodt, 2001; Makkar \& Strube, 1995). Other studies, however, failed to support the relationship between racial identity and eating disorders symptomatology (Akan \& Grilo, 1995; Edwards-Hewitt \& Gray, 1993). Again, inconsistent findings may be due to small effect size, low power, or slight variations in samples, terminology, or psychometric properties associated with the measures of racial identity attitudes. 
Ethnic identity and disordered eating. Ethnic identity is a multidimensional construct that includes a sense of belonging to a group, positive evaluation of the group, and participation in traditions and activities associated with the group (Phinney, 1992). Phinney (1996) proposed that ethnic identity is the link between an individual's ethnic background and psychological adjustment. Given Phinney's proposition, Abood and Chandler (1997) urged researchers to examine the role ethnic identity plays in the development of eating disorders and eating disorder symptomatology among members of various ethnic groups. As African American culture appreciates a diversity of body sizes and shapes and places less emphasis on achieving the thin-ideal than does European American culture (Choate, 2005; Powell \& Kahn, 1995), Striegel-Moore and Smolak (1996) postulated that degree of ethnic identity would be associated with body dissatisfaction and eating disorder symptomatology among African American women. Specifically, higher levels of identification with African American culture were thought to be associated with lower levels of body dissatisfaction and risk for eating disorder.

Using the Multi-Group Ethnic Identity Scale (Phinney, 1992), several studies (e.g., Baugh, 2005; Cachelin \& Regan, 2006; Petersons et al., 2000; Sabik et al., 2007) explored the relationship between ethnic identity and eating disorder symptomatology. Some of these studies found a relationship between ethnic identity and eating disorder symptomatology (i.e., dietary restraint) among African Americans. While exploring correlates of dietary restraint among a large, multi-ethnic, community-based sample of men and women, Cachelin and Regan (2006) found an inverse relationship between ethnic identity and dietary restraint. In another study, Sabik et al. (2007) recruited 86 African American, 178 Asian American, and 774 European American from 
undergraduate courses. Participants were administered the Multi-Group Ethnic Identity Measure (Phinney, 1992) to measure ethnic identity. In addition, participants were administered the Body Esteem Scale (Franzoi \& Shields, 1984) and Eating Disorder Inventory (Garner et al., 1983) to measure body image and eating disorder symptomatology, respectively. Results suggested that ethnic identity related to drive for thinness. Specifically, ethnic identity negatively related to drive for thinness among African Americans and positively related to drive for thinness among Asian American and European American women.

In contrast to the aforementioned studies (Cachelin \& Regan, 2006; Sabik et al., 2007), several studies (Baugh, 2005; Petersons et al., 2000) found only partial support or no support for a relationship between ethnic identity and eating disorder symptomatology. For example, Petersons et al. (2000) examined the relationship between eating disorder symptoms and ethnic identity with a nationwide sample of African American and European American college women. They administered demographic assessments, Eating Disorder Inventory-2 (Garner, 1991), and Multi-Group Ethnic Identity instruments (Phinney, 1992). Ethnic identity related to eating disorder symptoms (e.g., drive for thinness, bulimia, and body dissatisfaction) for European American, but not African American, participants. Specifically, higher levels of identification with European American culture were associated with higher levels of drive for thinness, bulimia, and body dissatisfaction. No relationship was found between ethnic identity and drive for thinness, bulimia, and body dissatisfaction among African American women. 
Similarly, Baugh (2005) recruited 45 African American and 70 European American women from two southeastern universities, one predominantly White and one historically Black; however, the author did not report specific background details of the African American or European American participants. Participants completed the Multigroup Ethnic Identity Measure (Phinney, 1992), the Body Dissatisfaction and Drive for Thinness subscales of the Eating Disorder Inventory-2 (Garner, 1991), and a demographic form. African American women reported lower levels of eating disorder behavior than did their European American counterparts; however, no relationship was found between ethnic identity and eating disordered behavior among African American or European American women.

Clearly, studies that explored the relationship between ethnic identity and eating disorder symptomatology yielded conflicting results. Some results (Cachelin \& Regan, 2006; Sabik et al., 2007) suggested that ethnic identity, as measured by the Multi-group Ethnic Identity Measure, was associated with restrictive eating (i.e., dietary restriction and drive for thinness), but other results (Baugh, 2005; Petersons et al., 2000) suggested that ethnic identity, as measured by the same measure, was not associated with eating disorder symptomatology (e.g., drive for thinness, body dissatisfaction, bulimia). Minor differences in study design, eating disorder instrument, or sample characteristics may be responsible for conflicting results.

These conflicting results found in the body of research that examines the relationship between ethnic identity and eating disorder symptomatology mirror findings in the larger body of literature that examines the relationship between identification with African American culture (e.g., acculturation, African self-consciousness, and racial 
identity) and body dissatisfaction/eating disorder symptomatology. It is important to note that this body of literature includes a variety of terms, instruments, and sample types (e.g., African Americans attending a historically Black college), making it difficult to make comparisons across studies and form definite conclusions.

Summary. Although the body of literature that explores the relationship between identification with African American culture and body dissatisfaction/eating disorder symptomatology is disjointed, several studies found a relationship between identification with African American culture and body dissatisfaction/eating disorder symptomatology. Specifically, higher levels of identification with African American culture often are reported to be associated with lower levels of disordered eating attitudes (e.g., drive for thinness), disordered eating behaviors (e.g., dietary restriction), and body dissatisfaction (Abrams et al., 1993; Cachelin \& Regan, 2006; Falconer \& Neville, 2000; Harris, 1995; Pumariega, 1986; Sabik et al., 2007; Spadafore, 2008).

Although research has established some relationship between identification with African American culture and negative body image and maladaptive eating attitudes and behaviors, no research to date has explored the association between identification with African American culture and body appreciation and intuitive eating. Exploring the relationship between identification with African American culture and body appreciation and intuitive eating moves forward the literature on African American eating behaviors and body image. In addition, exploring the relationship between identification with African American culture and body appreciation and intuitive eating answers the call of Lopez et al. (2006) to develop theoretical frameworks for positive constructs (e.g., body 
appreciation and intuitive eating) and explore those frameworks with diverse samples (e.g., African American college women).

When exploring positive constructs (e.g., body appreciation and intuitive eating) among diverse samples, Constantine and Sue (2006) urged researchers to consider cultural values, beliefs, and practices. In the literature, however, authors use a variety of terms (e.g., acculturation, assimilation) to discuss and measure African Americans' cultural values, beliefs, and practices. Among the abundance of terms, racial and ethnic identity emerged as most prominent in theoretical and empirical writings (Cokley, 2007). Racial identity and ethnic identity are similar constructs, but important distinctions exist in their definition and measurement (Cokley, 2007; Helms, 1996; Worrell \& GardnerKitt, 2006).

Racial identity is an individual's perception of him or herself as a member of a racial group, which shares a common heritage or history (Cokley, 2007). Numerous theories and measures have been created to conceptualize racial identity. For example, the Racial Identity Attitudes Scale for Blacks (RIAS-B; Parham \& Helms, 1981) and the Cross Racial Identity Scale (CRIS; Vandiver, Cross, Fhagen-Smith, Worrell, Swim, \& Caldwell, 2000) have been created to operationalize the original nigrescence model (Cross, 1971) and the expanded nigrescence model (Cross \& Vandiver, 2001), respectively. Ethnic identity is an individual's knowledge, commitment, and evaluation of his or her group membership (Phinney, 1992, 1996). The Multi-group Ethnic Identity Measure (MEIM; Phinney, 1992) was created to operationalize Phinney's conception of ethnic identity across a range of group memberships. 
Using the above-mentioned scales, authors have explored the relationship between racial and ethnic identity in African American adolescents (Worrell \& Gardner Kitt, 2006) and college students (Goodstein \& Ponterotto, 1997; Phelps, Taylor, \& Gerard, 2001; Worrell \& Gardner Kitt, 2006). Bivariate (Goodstein \& Ponterotto, 1997; Phelps, Taylor, \& Gerald, 2001; Worrell \& Kitt, 2006) and canonical correlation (Worrell \& Gardner Kitt, 2006) studies suggest that ethnic identity is modestly related to, but distinct from, racial identity; moreover, authors have urged researchers to use caution when selecting their terms and measures in multicultural research (Goodstein \& Ponterotto, 1997; Phelps, Taylor, \& Gerard, 2001; Worrell \& Gardner-Kitt, 2006).

Given that ethnic identity assesses salience and valence of group membership and racial identity assesses self-concept in response to a societal ideal, both ethnic and racial identity may be appropriate constructs to consider in research about eating behaviors. African American women with high levels of ethnic or racial identity might perceive others as accepting their body regardless of congruence with thin-ideal and encouraging them to maintain a healthy body size and not lose weight. Greater levels of perceived acceptance from others may encourage African American women to focus on internal experience rather than external appearance and appreciate their bodies.

\section{Summary}

Body appreciation and intuitive eating are aspects of positive body image and healthy eating behaviors, respectively (Avalos et al., 2005; Tylka, 2006), that merit further attention in the counseling psychology literature. Avalos and Tylka (2006) proposed a framework to explain factors that contribute to body appreciation and intuitive 
eating, and empirical investigations with samples of mostly White, middle class, college women revealed that the model provided an adequate fit to the data (Augustus-Horvath, 2006; Avalos \& Tylka, 2006). In addition, data from a small number of African American women provided preliminary evidence for the generalizability of the construct of intuitive eating and its relationship to other constructs in the model of intuitive eating (Avalos \& Tylka, 2006). Nevertheless, it was important to explore the model holistically with a larger sample of African American women.

In such a sample, however, model modifications may be important to consider. Authors (Choate, 2005; Harris, 1994; Molloy \& Herzberger, 1998) have identified protective factors (e.g., greater acceptance of a diversity of body shapes and sized, expanded definition of attractiveness, and presence of supportive peers) in the African American community that may promote positive body image and protect against eating disorder symptomatology. Researchers exploring body image and eating behaviors among African American women, then, would do well to consider participants’ identification with African American culture (e.g., ethnic/racial identity). To date, no research explored the association of intuitive eating or body appreciation with identification with African American culture therefore exploring a model of intuitive eating that includes consideration of ethnic and racial identity extends the body image and eating behavior literature.

\section{Purpose}

The purpose of this study was to examine a model of intuitive eating with a large sample of African American college women. In particular, the present study used path 
analysis to determine whether the model of intuitive eating forwarded by Avalos and Tylka (2006) provided an adequate fit to the data and whether the hypothesized paths were upheld with a sample of African American college women.

The second purpose of this study was to integrate culturally relevant variables (i.e., ethnic/racial identity) into the aforementioned model of intuitive eating (see Figure 2) and reanalyze the data, again with path analysis to determine whether the alternative model provided an adequate fit to the data and whether the hypothesized paths were upheld. Assuming the alternative model provided an adequate fit to the data, the indirect (mediated) effect of ethnic and racial identity on body function and body appreciation through perceived body acceptance by others was to be explored. The final purpose of this study was to make a direct comparison across models using Akaike's information criterion. 


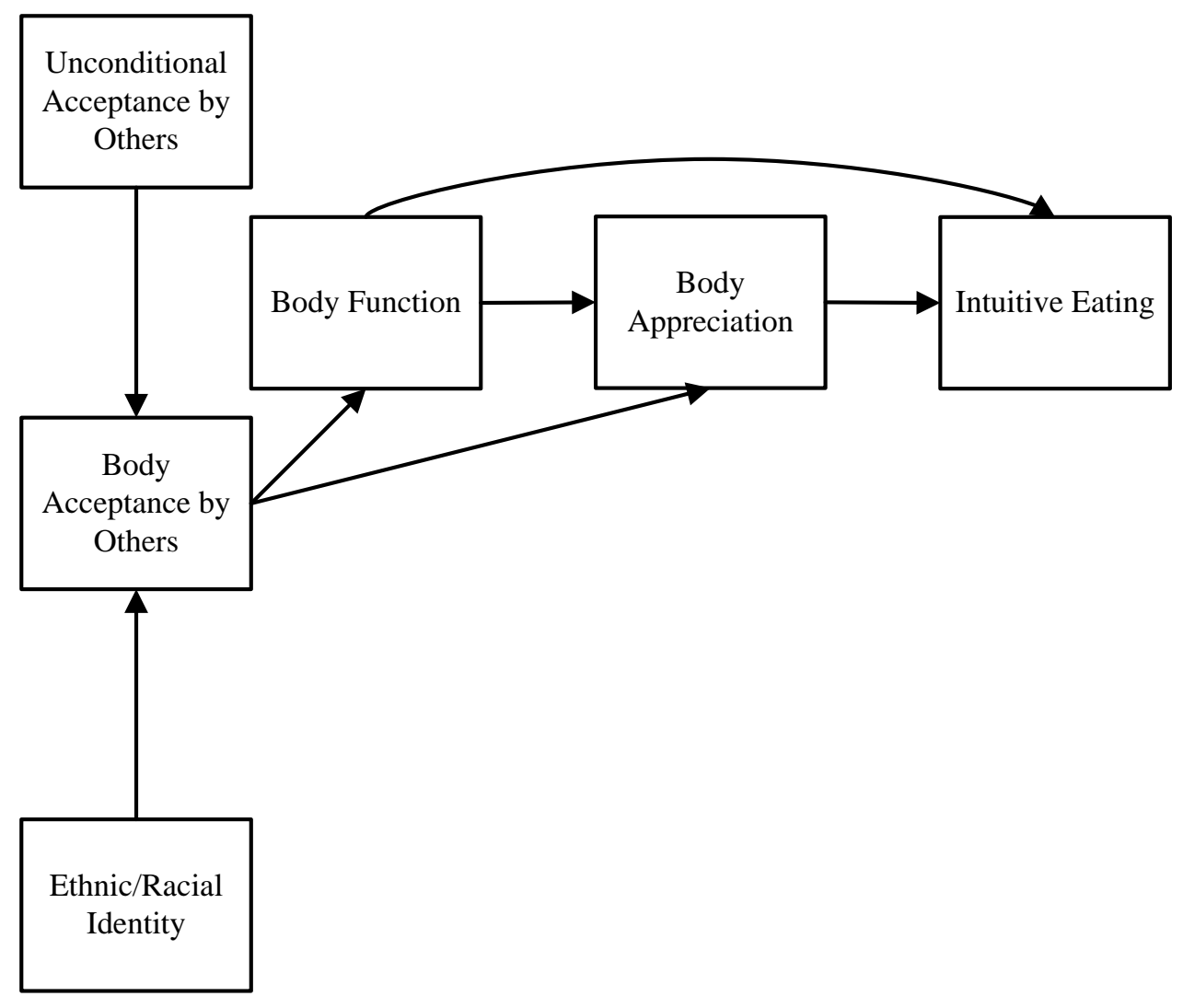

Figure 2. Proposed alternative model with culturally relevant variables (i.e., ethnic and racial identity).

\section{Hypotheses}

The present study explored a model of intuitive eating (Avalos \& Tylka, 2006) with a sample of African American college women. In addition, the present study integrated culturally relevant variables (i.e., ethnic/ racial identity) into Avalos and Tylka's model of intuitive eating and explored these culturally relevant alternative models with a sample of African American college women. The following hypotheses were forwarded: 
Hypothesis 1: The model of intuitive eating (Avalos \& Tylka, 2006) provides an adequate fit for the data obtained from a sample of African American college women.

Hypothesis 2: Paths proposed in the model of intuitive eating (Avalos \& Tylka, 2006) are upheld with data obtained from a sample of African American college women.

Hypothesis 2a: General acceptance from important others directly influences perceptions of body acceptance by others.

Hypothesis $2 \mathrm{~b}$ : Perceptions of body acceptance by others directly influence body function.

Hypothesis 2c: Perceptions of body acceptance by others directly influence body appreciation.

Hypothesis 2d: Body function directly influences intuitive eating. Hypothesis 2e: Body function directly influences body appreciation. Hypothesis 2f: Body appreciation directly influences intuitive eating. Hypothesis 2g: Body acceptance by others indirectly influences intuitive eating through body function and body appreciation.

Hypothesis 3: The culturally relevant alternative model provides an adequate fit to the data obtained from a sample of African American college women.

Hypothesis 4: Paths proposed in the culturally relevant alternative models are upheld with data obtained from a sample of African American college women. Hypothesis 4a: Ethnic/racial identity directly influences perceptions of body acceptance by others. 
Hypothesis 4b: General acceptance from important others directly influences perceptions of body acceptance by others.

Hypothesis 4c: Perceptions of body acceptance by others directly influence body function.

Hypothesis 4d: Perceptions of body acceptance by others directly influence body appreciation.

Hypothesis 4e: Body function directly influences intuitive eating. Hypothesis 4f: Body function directly influences body appreciation. Hypothesis 4g: Body appreciation directly influences intuitive eating. Hypothesis 4h: Ethnic/racial identity indirectly influences body function and body appreciation through body acceptance by others.

Hypothesis 4i: Body acceptance by others indirectly influences intuitive eating through body function and body appreciation.

Hypothesis 5: The culturally relevant alternative model provides a better fit to the data than the original model. 


\section{CHAPTER III}

\section{METHODS}

\section{Participants}

After obtaining Institutional Review Board approval, the researcher approached African American women at a large Midwestern university and offered the women and their friends an opportunity to participate in research on African American women's eating behaviors. Interested women received a survey packet and assurance that participation in this study was voluntary. Women were able to discard the survey packet without penalty.

An informed consent form was attached to the exterior of the survey packet. Inside the survey packet was a brief demographic form and seven research instruments. Interested women were instructed to return a completed survey packet to the researcher in a timely manner. In exchange for a completed packet, the researcher offered a small, cash award (i.e., \$5.00). The researcher maintained a payment log to discourage duplicate responses.

The researcher distributed 188 survey packets to African American women from undergraduate and graduate classes, African American student organizations, African American sororities, athletic activities, and public spaces (e.g., student union, food court, and library). It should be noted that a majority of the survey packets were distributed and 
returned to research participants outside of the food court during the noon hour. Of the 188 survey packets that were distributed, 147 packets were returned and 142 participants received a small cash award (i.e., \$5.00) for completing the survey packet. Five participants declined the small cash award.

Of the 147 packets returned, 130 were able to be used in the data analysis. Five women identified as "biracial" on the demographic forms, four women left more than 90\% of an instrument incomplete, three women identified as non-African American (e.g., Latina), and five women were less than 18 years old. As the study examined a model with 13 parameters, the sample size was large enough to meet the desired 10:1 parameterto-case ratio (Bentler, 1990).

Participants ranged in age from 18 to 54 years old $(\mathrm{M}=26.78, \mathrm{SD}=.81)$. Ten women chose not to disclose their age.

In terms of sexual orientation, 13 (12.3\%) of the African American women identified as exclusively homosexual, 10 (20\%) identified as bisexual, 14 (30.8\%) identified as primarily heterosexual, $87(66.9 \%)$ identified as exclusively heterosexual, 2 $(1.5 \%)$ identified as other, and $1(0.8 \%)$ identified as uncertain. Three African American female participants chose not to disclose their sexual orientation on the demographic questionnaire.

In terms of relationship status, $30(23.1 \%)$ of the African American women reported that they were not dating, 40 (30.8\%) reported that they were casually dating, 38 (29.2\%) reported that they were exclusively dating, $14(10.8 \%)$ reported that they were in a committed relationship (e.g., marriage, domestic partnership), and 7 (5.3\%) reported 
that they were apart from their partner (e.g., separated, divorced, or widowed). One African American college student chose not to disclose her relationship status.

In terms of college rank, $22(16.9 \%)$ of the African American college women were freshman, $26(20.0 \%)$ were sophomores, 25 (19.2\%) were juniors, and $32(24.6 \%)$ were seniors. In addition, $3(2.3 \%)$ of the African American women reported they were post-baccalaureate, $18(13.8 \%)$ were graduate students, and $3(2.3 \%)$ were other. One African American college student chose not to disclose her college rank

In terms of socioeconomic status, 21 (16.2\%) of these African American college women identified as lower class, $52(40.0 \%)$ identified as working class, $42(32.3 \%)$ identified as middle class, $11(8.5 \%)$ identified as upper-middle class, $1(.8 \%)$ identified as upper class, and $2(1.5 \%)$ identified as other. One African American college student woman chose not to disclose her socioeconomic status.

\section{Procedures}

Participants completed a demographic questionnaire and seven research instruments. To avoid unintentional priming, participants were presented with instruments in the following order: Intuitive Eating Scale, Body Appreciation Scale, Body Surveillance subscale of the Objectified Body Consciousness Scale, Body Acceptance by Others, Revised Barrett-Lennard Relationship Inventory, demographic questionnaire, Multi-Group Ethnic Identity Measure, and Internalization Afrocentricity subscale of the Cross Racial Identity Scale. This order is the reverse of the order in which the constructs are included in the model. 
Measures

\section{Demographic Sheet}

This is an author-composed seven item demographic questionnaire (see Appendix A) that requested participants to disclose personal information that included: sex, age, sexual orientation, ethnic identification, relationship status, year in school, and socioeconomic classification. Given the monetary incentive for participation in study and the author's concern that individuals who are not African American college women might attempt to participate, items assessing participant ethnic identification, sex, and year in school were included in the demographic questionnaire.

\section{General Unconditional Acceptance From Significant Others}

A revised version of the 36-item Barrett-Lennard Relationship Inventory (BarrettLennard, 1962; Claiborn et al., 1983) was used to assess African American women’s perceived general unconditional acceptance from significant others (see Appendix B).

Barrett-Lennard (1962) developed the Barrett-Lennard Relationship Inventory (BLRI) as an instrument for assessment in the person-centered model of psychotherapy. The original instrument had 64 items divided into four subscales (i.e., empathy, level of regard, unconditionality of regard, and congruence). Each item was scored on a six-point scale ranging from 1 (definitely true) to 6 (definitely not true). Half the items were framed in the direction of conditional acceptances and reversed scored. Researchers computed a total score and four subscale scores (Barrett-Lennard, 1962). 
Across a number of articles, support was garnered for the BLRI as an internally consistent and reliable measure of perceived unconditional acceptance (Simmons, Roberge, Kendrick, and Richards, 1995). Gurman (1977) conducted a review of studies on the BLRI and found support for internal consistency and test-retest reliability among samples of young, mostly White, men and women. Across 24 studies, Gurman calculated the mean internal consistency coefficients of BLRI subscale and total scores (i.e., empathy $\alpha=.84$, regard $\alpha=.91$, unconditionality of regard $\alpha=.74$, congruence $\alpha=.88$, total $\alpha=.91$ ). In addition, Gurman calculated the mean test-retest reliability coefficients of BLRI subscale and total scores (i.e., empathy $r=.83$, regard $r=.83$, unconditionality of regard $\mathrm{r}=.80$, congruence $\mathrm{r}=.85$, total $\mathrm{r}=.90$ ) across 10 studies.

Although Gurman (1977) found support for internal consistency and temporal stability of BLRI, support was not garnered for four distinct factors. In fact, Mills and Zytowski (1967) found intercorrelations between BLRI subscales and suggested the subscales are influenced by single factor. Given the intercorrelations among subscales, Avalos and Tylka (2006) and the current investigator opted to use only an averaged total score instead of four subscale scores. Item responses are averaged to obtain the averaged total score, with higher scores indicating greater levels of perceived unconditional acceptance by others. In the current study, the investigator calculated alpha to be .89 for the BLRI scores.

Mann and Murphy (1975) revised the Barrett-Lennard Relationship Inventory by reducing the number of items from 64 to 32 and adding four items to measure client resistance. Using this revised Barrett-Lennard Relationship Inventory, Avalos and Tylka (2006) and Wilcox (2006) provided initial support for the psychometric properties of the 
revised Barrett-Lennard Relationship Inventory among a sample of college women. Specifically, Avalos and Tylka (2006) calculated an internal consistency reliability coefficient of .84 and Wilcox (2006) found positive relationships between scores on the revised Barrett-Lennard Relationship Inventory and unconditional self-regard, psychological hardiness, and instrumentality. To date, no research has examined the Barrett-Lennard Relationship Inventory or revised Barrett-Lennard Relationship Inventory with a sample of African American college women.

The revised Barrett-Lennard Relationship Inventory was modified slightly for this study; instructions and items were adjusted to ask a participant to consider collectively the important others in her life as she grew up (i.e., "I have felt appreciated by him/her" was changed to "I have felt appreciated by them"). This alteration was undertaken to address limitations noted in previous research. Specifically, Avalos and Tylka (2006) assessed participants' perceived level of unconditional acceptance from the person in their youth that they deemed most important and influential. They later speculated that assessing perceived level of unconditional acceptance from only the most important other failed to capture overall level of perceived acceptance. For some women, "perhaps this most influential person did not give them the acceptance that they needed, but others in their environment were accepting of them" (Avalos \& Tylka, 2006, p. 496). Thus, the researcher slightly modified instructions and items to consider important others collectively and to thereby address the above-noted limitation and more broadly capture perceived unconditional acceptance.

Assessing level of perceived unconditional support from important others within one's early environment was especially relevant to African American women. When 
exploring positive constructs with African Americans, Constantine and Sue (2006) noted that the influence of extended family is imperative to consider. Broadly addressing perceived acceptance from important others incorporated the influence from extended family.

\section{Body Acceptance by Others}

The Body Acceptance by Others Scale was used to assess the Body Acceptance by Others construct (see Appendix C). Avalos and Tylka (2006) created The Body Acceptance by Others Scale by altering items on The Perceived Sociocultural Pressure Scale (PSPS).

Stice et al. (1996) developed the PSPS to assess degree of pressure women perceive to achieve and maintain a thin body from family, friends, dating partners, and the media. Specifically, the PSPS asks women to rate the amount of pressure they perceive from friends, family, dating partners, and the media to lose weight (e.g., I've felt pressure from to lose weight) and maintain a thin body (e.g., I've felt pressure from to have a thin body). Item ratings are averaged to determine the level of sociocultural pressure women perceive to have a thin body. Higher scores on the PSPS reflect greater perceived pressure from others and the media to achieve and maintain a thin body.

Using data from 125 young, mostly White women enrolled in high school or college courses, Stice et al. (1996) calculated a Cronbach alpha of .87 for the eight item PSPS. In the same research article, Stice et al. reported a .93 test-retest coefficient; this was obtained from a pilot study with 27 participants over a period of 2 weeks. Although 
Stice et al. reported the number of participants in their pilot study, they failed to report other relevant demographic information (e.g., gender, race, age).

Limited information is available on the validity of the PSPS. In fact, Stice et al. only alluded to the validity of the PSPS by saying, "Research with similar measures revealed strong correlation between child-reports of perceived pressure from parents to lose weight and parent-reports of pressure" (Stice et al., 1996, p. 538).

Instead of perceived sociocultural pressure to obtain and maintain a thin body, Avalos and Tylka (2006) were interested in assessing perceived body acceptance from others; however, no extant scale measured perceived body acceptance from others. Therefore, Avalos and Tylka (2006) created the Body Acceptance by Others Scale (BAOS) by altering the two core items that comprise the PSPS. Specifically, Avalos and Tylka (2006) changed the PSPS items to reflect perceived acceptance regarding body shape and size (e.g., I've felt acceptance from regarding my body shape and/or weight) and positive messages regarding the body (e.g., ___ has/have sent me the message that my body shape and weight are fine). In addition, they asked women to consider body acceptance from society, along with acceptance from family, friends, dating partners, and media. These two core items (i.e., I've felt acceptance from regarding my body shape and/or weight, ___ has/have sent me the message that my body shape and weight are fine) are rated for each of five sources (i.e., family, friends, dating partners, society, and media). A 5-point scale that ranges from never to always is used for the 10 ratings. Item ratings are averaged to determine perceived body acceptance from others. Higher scores on the BAOS reflect greater perceived body acceptance from a variety of information sources. 
Using pilot data from young, mostly White college women $(n=66)$, Avalos and Tylka (2006) found evidence of good internal consistency $(\alpha=.91)$, test-retest reliability $(\mathrm{r}=.85)$, and construct validity. Specifically, scores on the BAOS were associated inversely with perceived pressure for thinness $(r=-.69)$, but not related to impression management $(r=.07)$. Together, these results provide initial support for the BAOS as a reliable and valid measure of body acceptance from others.

Although research on the BAOS has been conducted exclusively on samples of young, mostly White, college women, the current study used the BAOS as a measure of perceived body acceptance from others with a sample of African American women enrolled in college. The author chose the BAOS for the present research because no other measure of body acceptance exists that has been examined among African American women, and because Avalos and Tylka (2006) and Augustus-Horvath (2008) both used the BAOS in their explorations of the Avalos and Tylka model of intuitive eating. In the present study, alpha was.85 for the BAOS scores.

\section{Body Function}

The Body Surveillance subscale of the Objectified Body Consciousness Scale (McKinley \& Hyde, 1996) was used to assess body function (see Appendix D). Using feminist theory and the experience of individual women, McKinley and Hyde (1996) rationally developed the Objectified Body Consciousness Scale (OBCS) to measure three aspects of objectified body consciousness: body surveillance, internalization of cultural body standards, and control beliefs. Across three studies with young, mostly White, college women, McKinley and Hyde (1996) found support for the three aspects of OBCS 
and evidence of internal consistency, test-retest reliability, and construct validity. Given evidence of sound psychometric properties, the OBCS and the subscales of OBCS (i.e., body surveillance, internalization of cultural body standards, and control belief) have been used in numerous research studies (e.g., Augustus-Horvath, 2008; Avalos et al., 2005; Avalos \& Tylka, 2006; Buchanan et al., 2008; Tylka \& Hill, 2004).

In this study, one subscale of the OBCS was used; specifically, the Body Surveillance subscale was used to assess Body Function among African American college women. The Body Surveillance subscale is composed of eight items rated on a seven-point scale from strongly disagree (1) to strongly agree (7). Items assess the degree to which a woman watches her body and emphasizes external appearance over internal experience. Six of the items on this scale are worded in the direction of body function (e.g., I think it is more important that my clothes are comfortable than that they look good on me) and two items are worded in the direction of body surveillance (e.g., I often worry about whether the clothes I am wearing make me look good).

In the standard scoring procedures, the six items worded in the direction of body function are reverse scored and added to the two items worded in the direction of body surveillance. Avalos and Tylka (2006) deviated from standard scoring procedures to obtain a measure of body function. In order to assess the degree to which a woman emphasizes internal experiences over external appearance, Avalos and Tylka (2006) reverse scored the two items worded in the direction of body surveillance and added them to the six items worded in the direction of body function. With all items worded in the direction of body function, items are averaged and higher scores on this scale demonstrate greater emphasis on internal experiences. With all items worded in the 
direction of body function, alpha was .86 for the OBCS. In this study, the same alternative scoring procedure was used to determine African American college women's level of body function and alphas was. 72 .

McKinley and Hyde (1996) provided evidence of internal consistency reliability, stability over 2 weeks, and construct validity for the OBCS. Specifically, they found moderate to high internal consistency reliability for body surveillance among samples of undergraduate $(\alpha=.89)$ women and their mostly middle-aged mothers $(\alpha=.76)$. In addition, McKinley and Hyde administered the OBCS to a sample of 103 undergraduate women and readministered the scale 2 weeks later. Across the two testing times, scores on body surveillance were highly correlated $(\mathrm{r}=.79)$. Body surveillance also correlated positively as expected with body shame $(r=.66)$, public self-consciousness $(r=.46)$, appearance orientation $(\mathrm{r}=.64)$, and disordered eating $(\mathrm{r}=.48)$; moreover, surveillance negatively correlated as expected with body esteem $(r=-.39)$ among young women. Buchanan et al. (2008) used the Surveillance subscale of the OBCS with a sample of 117 African American college women. They reported moderate internal consistency reliability $(\alpha=.70)$ and preliminary evidence of criterion-related validity; specifically, body surveillance correlated with skin-tone surveillance, a conceptually similar but distinct construct.

\section{Body Appreciation}

The Body Appreciation Scale (Avalos et al., 2005) was used to measure the body appreciation construct (see Appendix E). The Body Appreciation Scale (BAS) is a 13 item self-report scale that measures body appreciation. Body appreciation is a construct 
related to positive body image, and it reflects unconditional approval and respect of the body regardless of imperfections or congruence with societal standards of beauty or thinness. On the BAS, individuals rate items along a five point scale $(1=$ never, $2=$ seldom, 3 = sometimes, $4=$ often, 5 = always). Item ratings are averaged to obtain a body appreciation score. Higher scores reflect higher levels of body appreciation (Avalos et al., 2005).

Using theoretical writings on positive body image, Avalos et al. (2005) rationally developed 16 items for the BAS, but later deleted three items for redundancy. Using the 13 remaining items, Avalos et al. (2005) established the BAS as a psychometrically sound measure of body appreciation. Specifically, Avalos et al. (2005) provided support for a unidimensional factor structure, internal consistency, stability over time, and construct validity (i.e., convergent validity, discriminant validity, and incremental validity).

Using a common factor analysis with principal axis factoring, Avalos et al. (2005) found support for both a one and two-factor structure. Most items loaded equally on both factors; therefore, authors concluded that a unidimensional solution fit the data best. Using another sample of young, mostly White, college women, Avalos et al. (2005) used confirmatory factor analysis and supported the hypothesized unidimensional structure of the BAS. Specifically, fit indices (e.g., CFI, SRMR, RMSEA) suggested a one-factor solution provided adequate to excellent fit to data.

In addition to establishing the factor structure, Avalos et al. (2005) provided support for the construct validity of the BAS. Supporting the convergent validity of the BAS, BAS scores were associated positively with scores on measures of body esteem $(\mathrm{r}=$ 
$.50)$, self-esteem $(\mathrm{r}=.53)$, favorable appearance evaluation $(\mathrm{r}=.68)$, physical condition $(\mathrm{r}$ $=.60)$, and weight unconcern $(\mathrm{r}=.72)$. In addition, BAS scores were associated negatively with measures of body shame $(r=-.73)$, body surveillance $(r=-.55)$, body preoccupation $(\mathrm{r}=-.79)$, eating disorder symptomatology $(\mathrm{r}=-.60)$, and body dissatisfaction $(\mathrm{r}=-.73)$. Supporting its discriminant validity, BAS scores were unrelated to impression management $(\mathrm{r}=.14)$. Furthermore, BAS scores predicted criterion variables as expected (i.e., optimism, proactive coping, and self-esteem) above and beyond other indicators of body image (i.e., body esteem, body surveillance, and body shame).

Finally, Avalos et al. (2005) evaluated the internal consistency reliability and testretest reliability of the BAS. Among three samples of college women, internal consistency reliabilities were moderately high $(\alpha=.91, .93, .94)$ and among one sample of college women, BAS scores were stable over 3 weeks $(r=.90)$.

After Avalos et al. (2005) established the BAS as a psychometrically sound measure of body appreciation, researchers translated and explored the BAS with Austrian men and women (Swami, Stieger, Haubner, \& Voracek, 2008), British men and women (Swami, Hadji-Michael, \& Furnham, 2008) and Malaysian women (Swami \& ChamorroPremuzic, 2008). Swami, Stieger, Haubner, and Voracek (2008) and Swami, HadjiMichael, and Furnham (2008) suggested the BAS has good internal consistency and testretest reliability among diverse samples of men and women. In addition, results of these studies confirmed the unidimensional factor structure found in sample of young, mostly White, U.S. college women (Avalos et al., 2005). 
Among a sample of 591 Malaysian women, however, Swami and ChamorroPremuzic (2008) failed to support the unidimensional factor structure found in Western settings (Avalos et al., 2005; Swami, Stieger, Haubner, \& Voracek, 2008; Swami, HadjiMichael, \& Furnham, 2008). They conducted a principal components analysis and found support for a two-factor solution (i.e., General Body Appreciation and Body Image Investment). They noted that, "it seems unlikely that the two-factor structure is a statistical anomaly" (Swami \& Chamorro-Premuzic, 2008 p. 411); rather, it suggests the possibility of cross-cultural differences in body image. Given the possibility of a crosscultural difference in the factor-structure of the BAS, the current study included factor analysis to examine the dimensional structure of the BAS among the present sample of African American college women. In the current study, alpha was. 87 for the BAS.

\section{Intuitive Eating}

The Intuitive Eating Scale (Tylka, 2006) was used to assess the construct of intuitive eating (see Appendix F). The Intuitive Eating Scale (IES) assesses three components of intuitive eating: unconditional permission to eat (e.g., I try to avoid certain foods high in fat, carbohydrates, or calories), eating for physical rather than emotional reasons (e.g., I stop eating when I feel full), and reliance on internal hunger/satiety cues (e.g., I can tell when I am slightly full). Individuals rate agreement with items on a five point scale from 1 (strongly disagree) to 5 (strongly agree). Individual item ratings are averaged to obtain three subscale scores (i.e., Unconditional Permission to Eat, Eating for Physical Rather than Emotional Reasons, and Reliance on Internal Hunger/ Satiety Cues) 
and one total intuitive eating score. Higher scores reflect higher levels of intuitive eating. In the present research, the averaged total IES score was used.

Using extant writings on intuitive eating, adaptive eating, and unrestrained eating, Tylka (2006) developed 25 items to assess three core features of intuitive eating. Four of the original items loaded poorly (i.e., <.45) on their hypothesized latent factor and were subsequently deleted from the scale. Using a 21-item version of the IES, Tylka conducted a confirmatory factor analysis to ensure the model provided a good fit to the data. As hypothesized, a three factor model provided an adequate fit to the data (CFI = $.91, \mathrm{TLI}=.90, \mathrm{RMSEA}=.08, \mathrm{SRMR}=.07)$. In addition, items loaded on their respective first-order factors (i.e., Unconditional Permission to Eat, Eating for Physical Rather than Emotional Reasons, and Reliance on Internal Hunger/ Satiety Cues) and firstorder factors loaded on the second-order intuitive eating factor (Tylka, 2006).

Authors (Tylka, 2006; Wilcox \& Tylka, 2006) have provided support for the construct validity of the IES. Intuitive Eating Scale scores were strongly related to life satisfaction $(\mathrm{r}=.41)$ and self-esteem $(\mathrm{r}=.44)$ and moderately related to optimism $(\mathrm{r}=$ $.29)$ and proactive coping $(r=.29)$. In addition, IES scores related only negligibly $(r=$ .12) to impression management, a conceptually unrelated construct.

Finally, Tylka (2006) garnered preliminary evidence for the internal consistency and temporal stability of the IES. Tylka reported internal consistency reliability coefficients ranging from .72 to .87 and item-total correlations above .30 . Tylka also administered the IES to a sample of young, mostly White, college women and readministered the same instrument 3 weeks later. Over the 3-week period, IES total and subscale scores were consistent. Correlations ranged from .74 on the Reliance on 
Hunger/ Satiety Cues subscale to .90 for the total IES score (Tylka, 2006). In the current study, alpha was .84 for the IES.

\section{Ethnic Identity}

The Ethnic Identity subscale of The Multigroup Ethnic Identity Measure (Phinney, 1992) was used to assess the construct of ethnic identity (see Appendix G). Phinney developed items for The Multigroup Ethnic Identity Measure (MEIM) rationally from the Objective Measure of Ego Identity Status (Adams, Bennion, \& Huh, 1987) and interview studies of ethnic identity. The MEIM was developed to measure ethnic identity across diverse ethnic groups residing in the United States of America.

The MEIM is divided into three interrelated parts: Ethnic Identity, Other-Group Orientation, and Self-Identification. The Ethnic Identity subscale consists of 14 items that assess three aspects of ethnic identity: Affiliation and Belonging (e.g., I am happy that I am a member of the group I belong to), Ethnic Identity Achievement (e.g., I have spent time trying to figure out my own ethnic group, such as its history, traditions, and customs), and Ethnic Behaviors (e.g., I am active in organizations or social groups that include mostly members of my own ethnic group). Although these items assess three aspects of ethnic identity, factor analysis suggests these three aspects of ethnic identity are not distinct and a one-factor solution fits them best (Phinney, 1992).

Individuals rate their agreement with items on a four point scale that ranges from 1 (strongly disagree) to 4 (strongly agree). An ethnic identity score is obtained by reversing two negative items (i.e., I am not very clear about the role of my ethnicity and my life and I really have not spent much time trying to learn more about the culture and 
history of my ethnic group) and summing and then averaging across items. Higher scores reflect higher levels of ethnic identity. In the current investigation, the averaged total score on the Ethnic Identity subscale of the MEIM was used.

Part 2 of the MEIM consists of six items that assess Other-Group Orientation (e.g., I like meeting and getting to know people from ethnic groups other than my own). Again, individuals rate agreement with items on a four point scale that may range from 1 (strongly disagree) to 4 (strongly agree). Other-Group Orientation scores are obtained by summing items. Higher scores reflect higher levels of other group orientation.

Part 3 of the MEIM consists of three items that assess Self-Identification (e.g., My ethnicity is ). Responses to these three open-ended questions are not scored.

A number of authors (Cuellar, Arnold, Maldonado, 1995; Goodstein \& Ponterotto, 1997; Phinney, 1992; Phinney, Chavira, \& Tate, 1996; Ponterotto, Gretchen, Utsey, Stracuzzi, \& Saya, 2003; Worrell, 2000) provided support for the MEIM's factor structure, construct validity, internal consistency reliability, and test-retest reliability. Using the responses from 417 ethnically diverse high school students and 136 ethnically diverse college students, Phinney (1992) provided initial support for two relatively independent factors of the MEIM (i.e., Ethnic Identity and Other Group Orientation). Worrell (2000) and Ponterotto et al. (2003) replicated and extended the work of Phinney (1992) by conducting confirmatory factor analyses using data from adolescents (10-18 years old). Results of both confirmatory factor analyses supported a two-factor solution (i.e., Ethnic Identity and Other Group Orientation) similar to that reported by Phinney (1992). 
Authors (e.g., Phinney, 1992; Ponterotto et al., 2003; Worrell, 2000) also have examined the construct validity of MEIM scores. For example, Phinney (1992) administered the MEIM and the 10-item Rosenberg Self-Esteem Inventory (Rosenberg, 1965) to ethnically diverse high school and college students and found a moderate association between MEIM scores and self-esteem among high school $(r=.31)$ and college students $(r=.25)$. Subsequent authors confirmed the moderate association between MEIM and self-esteem (Goodstein \& Ponterotto, 1997) and established theoretically consistent relationships between MEIM scores and racial identity development scores (Goodstein \& Ponterotto, 1997), acculturation (Cuellar et al., 1995), and ethnic self-concept (Phinney et al., 1996).

Finally, authors (e.g., Birnbaum, 1991; Phinney, 1992) have calculated internal consistency coefficients for the two MEIM subscales (i.e., Ethnic Identity and Other Group Orientation). Among samples of ethnically diverse, high school and college students, internal consistency coefficients ranged from .81 (Phinney, 1992) to .92 (Birnbaum, 1991; Taub, 1995) on the Ethnic Identity subscale and from .35 (Ponterotto, Baluch, Greig, \& Rivera, 1998) to .82 (Taub, 1995) on the Other Group Orientation subscale of the MEIM. In the current study, alpha was .84 for the Ethnic Identity subscale of the MEIM.

\section{Racial Identity}

The Internalization Afrocentricity subscale of the Cross Racial Identity Scale (Vandiver, Cross, Fhagen-Smith, Worrell, Swim, \& Caldwell, 2000) was used to assess racial identity (see Appendix H). Over a 5-year period, Vandiver et al. developed the 
Cross Racial Identity Scale (CRIS) to operationalize the expanded nigrescence (NT-E) model (Cross \& Vandiver, 2001) and overcome psychometric problems publicized in the research literature of measures of racial identity attitudes (Worrell \& Watson, 2008).

Cross and Vandiver (2001) developed the NT-E as an update to the original (Cross, 1971) and revised (Cross, 1991) nigresence model. Moreover, the NT-E presents a series of racial identity attitudes organized under three themes (i.e., preencounter, immersion-emersion, and internalization). The preencounter theme includes three racial identity attitudes (i.e., assimilation, miseducation, and self-hatred) and represents an unfavorable view of African Americans and African American culture. The immersionemersion theme includes two racial identity attitudes (i.e., anti-White and intense Black involvement) and represents unwavering, positive views for African Americans and African American culture and negative views for European Americans and European American culture. Finally, the internalization theme includes three racial identity attitudes (i.e., afrocentricity, bicultural, and multicultural) and represents appreciation for African American heritage. Although identities under the internalization theme recognize African American culture, they do not prevent self or others from recognizing other identities (Cross \& Vandiver, 2001; Vandiver et al., 2000; Worrell, Vandiver, \& Cross, 2004; Worrell \& Watson, 2008).

Although the NT-E includes eight racial identity attitudes organized under three themes, the CRIS assesses only six racial identity attitudes under three themes. Immersion-emersion intense Black involvement and internalization bicultural attitudes were intentionally excluded from the current version of the CRIS. Items that assess internalization of intense Black involvement are under development and should be 
included in future versions of the CRIS (Vandiver, Cross, Worrell, \& Fhagen-Smith, 2002). Although future versions of the CRIS should include items that assess internalization intense Black involvement, the authors do not anticipate the inclusion of items that measure internalization bicultural attitudes in a future version. The authors noted that the presence of numerous possible bicultural attitudes makes the development and content validation of such a scale extremely difficult (Vandiver et al., 2002).

At present, the CRIS contains six subscales that assess three preencounter attitudes (i.e., assimilation, miseducation, \& self-hatred), one immersion-emersion attitude (i.e., anti-White), and two internalization attitudes (i.e., afrocentricity \& multiculturalist).

Across a number of development and validation studies, various authors found support for a six-factor model (Helms, 2002; Gardner-Kitt \& Worrell, 2007; Worrell et al., 2004; Worrell \& Watson, 2008), internal consistency (Cokley, 2002; Helms, 2002; Gardner-Kitt \& Worrell, 2007; Vandiver et al., 2002; Worrell et al., 2004), and construct validity (Vandiver et al., 2002; Worrell \& Gardner-Kitt, 2006) of the CRIS. Given evidence of sound psychometric properties, the CRIS and CRIS subscales (i.e., PreEncounter Assimilation, Pre-Encounter Miseducation, Pre-Encounter Self-Hatred, Immersion-Emersion Anti-White, Internalization Afrocentricity, and Internalization Multiculturalist) have been used in research studies which examine the relation of racial identity attitudes to the psychological functioning of African Americans.

In this study, one subscale of the CRIS was used; specifically, the Internalization Afrocentricity (IA) was used to assess racial identity among African American college women. The IA subscale is composed of five items rated on a seven-point scale from 
strongly disagree (1) to strongly agree (7). Items assess the degree to which African Americans use Afrocentric beliefs and values as a standard for living. Items responses were averaged to obtain a subscale score with higher scores indicating greater adherence and utilization of Afrocentric beliefs and values.

Authors have provided evidence of internal consistency reliability and construct validity for the IA. Worrell and Watson (2008) reported reliability estimates (i.e., Cronbach's alpha) for the IA from ten studies. A visual examination of the ten reliability estimates suggests that the IA subscale is internally consistent among samples of African Americans. Specifically, coefficient alpha reliability estimates for this subscale ranged from .80 (Jones, Cross \& DeFour, 2007; Gardner-Kitt \& Worrell, 2007) to .85 (Worrell, Vandiver, \& Cross, 2004). Median internal consistency reliability across the ten studies was .83 (Worrell \& Watson, 2008).

In addition to establishing the internal consistency, authors provided support for the construct validity (i.e., convergent and discriminant validity) of the IA. Supporting the convergent validity of the IA, Afrocentricity scores were associated positively with scores on a measure of ethnic identity (Worrell \& Gardner-Kitt, 2006). In addition, IA scores were unrelated to impression management, self-esteem, and Big Five personality traits (i.e., neuroticism, extroversion, agreeableness), and associated negatively with measures of body shame (Vandiver et al., 2002); moreover, the lack of significant association between IA scores and impression management, self-esteem, and personality traits provides support for discriminant validity of the IA. In the current investigation, alpha was .84 for IA subscale of the CRIS. 


\section{CHAPTER IV}

\section{RESULTS}

Factor Analysis of Body Appreciation Scale

The responses from 130 African American college women were entered into SPSS and data analysis procedures contained in SPSS were used to describe the data and its appropriateness for factor and path analyses.

Mean, standard deviation, skewness, and kurtosis values were calculated for each measure and they are depicted in Table 1. Slight deviations from normality (e.g., skewness and kurtosis) were detected for the Body Appreciation Scale and Ethnic Identity subscale of the Multigroup Ethnic Identity Scale. The researcher then examined the skewness statistic in conjunction with standard error and determined that observed values exceeded standard cutoff scores. Significant Kolmogorov-Smirnov statistics confirmed above-noted deviation from normality.

Given the slight deviation from normality, a box plot was generated in SPSS and data were screened for outliers. Four outliers were detected. When the four outliers were excluded from analysis, no substantial violations to normality were indicated. Given the presence and impact of four outliers, data analyses were conducted with the outliers included and excluded from data set. Similar results were obtained with and without outliers included in the data set. For brevity, results from data analysis with outliers 
included are reported in this chapter. Results from data analysis with outliers excluded are reported in Appendix I for the reader's consideration.

Table 1

Means, Standard Deviations, Skewness, and Kurtosis for Measures With Responses of 130 African American College Women

\begin{tabular}{|c|c|c|c|c|c|c|}
\hline & Minimum & Maximum & $M$ & $S D$ & Skewness & Kurtosis \\
\hline $\begin{array}{l}\text { Barrett- } \\
\text { Lennard } \\
\text { Relationship } \\
\text { Inventory }\end{array}$ & 2.03 & 5.56 & 4.35 & .678 & -.547 & .020 \\
\hline $\begin{array}{l}\text { Body } \\
\text { Acceptance } \\
\text { by Others } \\
\text { Scale }\end{array}$ & 1.50 & 5.00 & 3.75 & .759 & -.311 & -.173 \\
\hline $\begin{array}{l}\text { Body } \\
\text { Surveillance } \\
\text { subscale of } \\
\text { OBC Scale }\end{array}$ & 1.13 & 5.50 & 3.50 & .848 & -.172 & -.045 \\
\hline $\begin{array}{l}\text { Body } \\
\text { Appreciation } \\
\text { Scale }\end{array}$ & 1.69 & 5.00 & 3.88 & .637 & -.837 & 1.077 \\
\hline $\begin{array}{l}\text { Intuitive } \\
\text { Eating Scale }\end{array}$ & 1.67 & 4.76 & 3.32 & .567 & -.155 & -.041 \\
\hline $\begin{array}{l}\text { Ethnic } \\
\text { Identity } \\
\text { subscale of } \\
\text { MEIM }\end{array}$ & 1.93 & 4.00 & 3.33 & .430 & -.576 & -.243 \\
\hline $\begin{array}{l}\text { Internalization } \\
\text { Afrocentricity } \\
\text { subscale of } \\
\text { CRIS }\end{array}$ & 1.00 & 6.40 & 3.40 & 1.18 & .088 & -.112 \\
\hline
\end{tabular}

Correlations among all variables were calculated and are depicted in Table 2.

Most correlations were positive and small (i.e., less than .23) to medium (i.e., between

.23 and .36) in size, as defined by Cohen (1992). Although most correlations were small 
to medium, the relationships between perceived body acceptance by others and body appreciation, perceived body acceptance by others and intuitive eating, body appreciation and body surveillance, and body appreciation and intuitive eating were positive and large in size whereas the relationship between unconditional acceptance and racial identity was negative and large in size (i.e., greater than .36).

Table 2

Correlations Among the Measures for Responses of 130 African American College Women

\begin{tabular}{|c|c|c|c|c|c|c|c|}
\hline & 1 & 2 & 3 & 4 & 5 & 6 & 7 \\
\hline $\begin{array}{l}\text { 1. Barrett- } \\
\text { Lennard } \\
\text { Relationship } \\
\text { Inventory }\end{array}$ & - & $.175^{*}$ & .059 & $.240 * *$ & $.324 * *$ & .024 & $-.440 * *$ \\
\hline $\begin{array}{l}\text { 2. Body } \\
\text { Acceptance by } \\
\text { Others Scale }\end{array}$ & & - & .154 & $.515 * *$ & $.424 * *$ & .016 & -.049 \\
\hline $\begin{array}{l}\text { 3. Body } \\
\text { Surveillance } \\
\text { subscale of } \\
\text { OBC Scale }\end{array}$ & & & - & $.383 * *$ & $.284 * *$ & -.101 & -.128 \\
\hline $\begin{array}{l}\text { 4. Body } \\
\text { Appreciation } \\
\text { Scale }\end{array}$ & & & & - & $.565^{* *}$ & .134 & -.017 \\
\hline $\begin{array}{l}\text { 5. Intuitive } \\
\text { Eating Scale }\end{array}$ & & & & & - & -.014 & -.196 \\
\hline $\begin{array}{l}\text { 6. Ethnic } \\
\text { Identity } \\
\text { subscale of } \\
\text { MEIM }\end{array}$ & & & & & & - & .162 \\
\hline $\begin{array}{l}7 . \\
\text { Internalization } \\
\text { Afrocentricity } \\
\text { subscale of } \\
\text { CRIS }\end{array}$ & & & & & & & - \\
\hline
\end{tabular}


In anticipation of the planned factor analysis of the Body Appreciation Scale, means, standard deviations, and correlations among items on the Body Appreciation Scale were calculated and are presented in Table 3. Most inter-item correlations were positive and large in size, as defined by Cohen (1992), but items 9 and 12 demonstrated modest correlations with other items on the scale.

As previous support has been found for both a one and two-factor structure of the Body Appreciation Scale, multiple one and two-factor models were specified and tested to determine the best fitting model with data obtained from African American college women.

Rogers, Abbey-Hines, and Rando (1997) suggested that multiple models be specified and tested with a variety of goodness-of-fit indices to determine the best fitting model. Thus, the following goodness-of-fit indices were utilized for both the factor and path analyses: Chi-squared significance test, Chi-square/df ratio, Comparative Fit Index (CFI), Tucker Lewis Index (TLI), Standardized Root Mean Square Residual (SRMR) and Root Mean Square Error of Approximation (RMSEA) with 90\% confidence interval. 
Table 3

Mean, Standard Deviation, and Correlations Among Items on the Body Appreciation Scale With Responses of 130 African American College Women

\begin{tabular}{|c|c|c|c|c|c|c|c|c|c|c|c|c|c|}
\hline & 1 & 2 & 3 & 4 & 5 & 6 & 7 & 8 & 9 & 10 & 11 & 12 & 13 \\
\hline 1 & 1 & $.561 * *$ & $.419 * *$ & $.565^{* *}$ & $.454 * *$ & $.543^{* *}$ & $.629 * *$ & $.266^{* *}$ & .137 & $.520 * *$ & $.411 * *$ & .149 & $.526^{* *}$ \\
\hline 2 & & 1 & $.682 * *$ & $.601 * *$ & $.393 * *$ & $.604 * *$ & $.472 * *$ & $.193 *$ & .075 & $.625^{* *}$ & $.377 * *$ & .143 & $.559 * *$ \\
\hline 3 & & & 1 & $.699 * *$ & $.414^{* *}$ & $.623^{* *}$ & $.363^{* *}$ & $.208 *$ & .082 & $.697 * *$ & $.400 * *$ & $.206^{*}$ & $.594 * *$ \\
\hline 4 & & & & 1 & $.541 * *$ & $.739 * *$ & $.471 * *$ & $.340 * *$ & .107 & $.720 * *$ & $.344 * *$ & $.242 * *$ & $.694 * *$ \\
\hline 5 & & & & & 1 & $.621 * *$ & $.442 * *$ & .156 & -.003 & $.543^{* *}$ & $.299 * *$ & .149 & $.627 * *$ \\
\hline 6 & & & & & & 1 & $.549 * *$ & $.350 * *$ & .061 & $.759 * *$ & $.459 * *$ & .157 & $.716^{* * *}$ \\
\hline 7 & & & & & & & 1 & $.245^{* *}$ & .019 & $.487 * *$ & $.440 * *$ & .091 & $.470 * *$ \\
\hline 8 & & & & & & & & 1 & .094 & $.298^{* *}$ & $.265^{* *}$ & $.302 * *$ & $.308 * *$ \\
\hline 9 & & & & & & & & & 1 & .102 & .026 & .164 & .154 \\
\hline 10 & & & & & & & & & & 1 & $.455^{* *}$ & .142 & $.761 * *$ \\
\hline 11 & & & & & & & & & & & 1 & .103 & $.309 * *$ \\
\hline 12 & & & & & & & & & & & & 1 & $.196 *$ \\
\hline 13 & & & & & & & & & & & & & 1 \\
\hline$M$ & 4.38 & 3.74 & 3.72 & 4.05 & 4.41 & 4.02 & 3.98 & 3.74 & 3.14 & 3.88 & 3.66 & 3.45 & 4.32 \\
\hline$S D$ & 0.77 & 0.97 & 1.00 & 0.98 & 0.83 & 0.95 & 0.92 & 1.25 & 1.03 & 0.96 & 0.90 & 1.46 & 0.94 \\
\hline
\end{tabular}

*Correlation is significant at the 0.05 level. ${ }^{* *}$ Correlation is significant at the 0.01 level. 
A nonsignificant chi-square statistic indicates that the model provides a good fit to the data, but sample size heavily influences chi-square results. With large samples, chisquared reaches significance and indicates a poor fit to the data, even when other indices suggest an adequate to excellent fit (Todman \& Dugard, 2007). Therefore, authors (Bryne, 2001; Hu \& Bentler, 1999; Rogers et al., 1997) suggest examining the significance of chi-squared and other indicators of model-data fit. In addition to examining the significance of chi-squared, chi-squared can be examined in conjunction with degrees of freedom to get a rough goodness-of-fit estimate. A chi-squared/df ratio less than 2 suggests that the model provides a good fit to the data.

The Tucker Lewis Index (TLI) and Confirmatory Fit Index (CFI) examine the extent to which a specified model accounts for sample covariance. Values of one on the TLI and CFI indicate a perfect model-data fit. Values equal to or greater than .95 on the TLI and CFI indicate an excellent model-data fit. Values between .90 and .94 on the TLI and CFI indicate an adequate model-data fit. Finally, values equal to or less than .90 on the TLI and CFI indicate a poor model-data fit (Hu \& Bentler, 2000; Rogers et al., 1997).

The Standardized Root Mean Square Residual (SRMR) measures the average covariance residual assuming that all variables were standardized prior to the analysis. Root Mean Square Error of Approximation (RMSEA) with 90\% confidence interval measures the difference between the specified model and the data for each degree of freedom in the model. Values of zero on the SRMR and RMSEA indicate a perfect model-data fit. Values equal to or less than .05 on the SRMR and RMSEA indicate an 
excellent model-data fit. Values between .06 and .10 on the SRMR and RMSEA indicate an adequate model-data fit. Finally, values equal to or greater than .10 on the SRMR and RMSEA indicate a poor model-data fit (Hu \& Bentler, 2000; Rogers et al., 1997).

First, a one-factor model was specified for which the 13 items on the Body Appreciation Scale served as indicators for the body appreciation latent variable. The model provided an adequate fit to the data $\left(\chi^{2}=125, \chi^{2} / d f=1.93, \mathrm{CFI}=.905, \mathrm{TLI}=.887\right.$, SRMR $=.068, \mathrm{RMSEA}=.086)$ and twelve of the thirteen indicators (i.e., all except Item 9) loaded significantly on the body appreciation latent variable. Two indicators (i.e., Items 8 \& 12) loaded relatively low on the latent variable body appreciation. Item loadings ranged from .115 to .868 .

Although the one-factor model provided an adequate fit to the data, three items had relatively poor loadings on the latent variable body appreciation, therefore a second one-factor model with items 8,9 , and 12 excluded was analyzed. The one-factor model with items 8,9 , and 12 excluded provided an unacceptable fit to the data $\left(\chi^{2}=92.2, \chi^{2} / d f\right.$ $=2.63, \mathrm{CFI}=.909, \mathrm{TLI}=.883, \mathrm{SRMR}=.046, \mathrm{RMSEA}=.114)$.

Because item 8,9 , and 12 provided low factor loadings in the one-factor model and model-data fit was poor when the above-noted items were removed, a third onefactor model was explored. In the third one-factor model, thirteen items served as indicators for the body appreciation latent variable and error terms associated with items 8,9 , and 12 were allowed to correlate. Item loadings ranged from .111 to .871 . Twelve of the thirteen indicators (i.e., all except Item 9) loaded significantly on the body 
appreciation latent variable. Two indicators (i.e., items $8 \& 12$ ) loaded relatively low on the latent variable. The correlation between error terms associated with items 8 and 9 was .059.

Fit indices for the one-factor model with error terms associated with items 8,9 , and 12 allowed to correlate $\left(\chi^{2}=115, \chi^{2} / d f=1.86, \mathrm{CFI}=.917, \mathrm{TLI}=.896, \mathrm{SRMR}=\right.$ .047 , RMSEA $=.083)$ suggested a better model-data fit than that for a one-factor model with uncorrelated error terms and a one-factor model with items 8, 9, and 12 deleted. Given the subtle improvement in model-data fit, a final one-factor model with error terms associated with items 8 and 12 allowed to correlate was explored. No significant difference was observed between the one-factor model with error terms associated with items 8,9 , and 12 allowed to correlate and the one-factor model with error terms associated with 8 and 12 allowed to correlate $\left(\chi^{2}=117, \chi^{2} / d f=1.83\right.$, CFI $=.917$, TLI $=$ .899, SRMR $=.051$, RMSEA $=.082$ ); therefore, the most parsimonious model (onefactor model with error terms associated with items 8 and 12 allowed to correlate) was determined to have the best fit to the data.

Considering the relatively poor loadings of three items in conjunction with previous empirical support suggesting a two-factor structure (Swami \& ChamorroPremuzic, 2008), two subsequent confirmatory factor analyses were conducted that examined a two-factor model. Based on factor loadings and previous research, a twofactor uncorrelated model was specified where items $1,2,3,4,5,6,7,10,11$, and 13 served as indicators for body appreciation latent variable and items 8,9 , and 12 served as indicators 
of a body image investment latent variable. Results demonstrated that the two-factor uncorrelated model provided an unacceptable fit to data, as most fit indices ranged from adequate to poor $\left(\chi^{2}=125, \chi^{2} / d f=1.92, \mathrm{CFI}=.907, \mathrm{TLI}=.888, \mathrm{SRMR}=.091, \mathrm{RMSEA}\right.$ $=.086)$.

A two-factor model with body acceptance and body image investment latent variables that were allowed to covary was examined next. Most model fit statistics indicated that the two-factor model provided an acceptable fit to the data, as fit indices ranged from adequate to excellent $\left(\chi^{2}=116, \chi^{2} / d f=1.82, \mathrm{CFI}=.919, \mathrm{TLI}=.901, \mathrm{SRMR}\right.$ $=.049, \mathrm{RMSEA}=.081)$. Confirmatory factor analyses with 130 African American college women, thus, provided support for a two-factor solution (e.g., body appreciation and body image investments) in which body appreciation and body image investment are correlated.

Collectively results of confirmatory factor analyses provided support for both a one-factor model with error terms associated with items 8 and 12 allowed to correlate and a two-factor model in which body appreciation and body image investment are correlated. As support was garnered for the one and two-factor solution, the most parsimonious model (one-factor model) was utilized in this investigation. All thirteen items of the Body Appreciation Scale were utilized as indicators of the latent variable body appreciation. For the reader's consideration, statistical results of confirmatory factor analysis and pictorial depictions of one and two-factor models are presented in Appendix J. 


\section{Path Analysis}

\section{Original Model With Outlier Cases Included in Data Analysis}

In order to determine whether the original model of intuitive eating (Avalos \& Tylka, 2006) provides an adequate fit to the data from African American college women, the researcher utilized path analysis with a maximum likelihood (ML) method of estimation and the covariance matrix supplied as input. Averaged total scores from the instruments served as indicators of observed variables in the path models. Moreover, adequacy of fit was determined with the above noted goodness-of-fit indices (i.e., chisquared significance test, chi-square/df ratio, CFI, TLI, RMSR, and RMSEA with 90\% confidence interval).

The model provided an adequate to poor fit to the data from this sample of 130 African American college women. As the model provided an adequate to poor fit to the data, support was not garnered for Hypothesis 1. Results suggest that the model of intuitive eating (Avalos \& Tylka, 2006) did not fit this sample of 130 African American college women. As seen in Table 4, the chi-squared statistic was significant, the chisquared/df ratio was 4.096, and the CFI (.898), TLI (.745), SRMR (.035), and RMSEA (.155) indicated only adequate to poor fit. Four of the six proposed paths, however, were significant at the .05 level. Specifically, paths from general acceptance to perceptions of body acceptance by other, perception of body acceptance by others to body appreciation, body function to body appreciation, and body appreciation to intuitive eating were 
significant. These significant paths provide support for hypotheses $2 \mathrm{a}$ (i.e., general acceptance from important others directly influences perceptions of body acceptance by others, 2c (i.e., perceptions of body acceptance by others directly influence body appreciation), 2e (i.e., body function directly influences body appreciation), and $2 \mathrm{f}$ (i.e., body appreciation directly influences intuitive eating) despite the lack of support for the overall model. Nonsignificant paths from perceptions of body acceptance by others to body function and body function to intuitive eating failed to support hypotheses $2 b$ (i.e., perceptions of body acceptance by others directly influences body function) and $2 \mathrm{~d}$ (i.e., body function directly influences intuitive eating). As four of the six proposed paths were significant at the .05 level, Hypothesis 2 was partially supported. Path coefficients for the model are presented in Figure 3. 
Table 4

Goodness-of-Fit Indices for Path Analyses for Responses From 130 African American College Women

\begin{tabular}{|c|c|c|c|c|c|c|c|c|c|c|c|}
\hline Model & $\chi^{2}$ & $d f$ & $\chi^{2} / d f$ & $p$ & CFI & TLI & RMSR & RMSEA & \multicolumn{2}{|c|}{$90 \% \mathrm{CI}$} & AIC \\
\hline Original & 16.383 & 4 & 4.096 & .003 & .898 & .745 & .035 & .155 & .082 & .236 & 38.383 \\
\hline $\begin{array}{l}\text { Culturally } \\
\text { Relevant } \\
\text { Alt- Racial } \\
\text { Identity }\end{array}$ & 50.893 & 8 & 6.362 & .000 & .716 & .468 & .093 & .204 & .152 & .259 & 76.893 \\
\hline $\begin{array}{l}\text { Culturally } \\
\text { Relevant } \\
\text { Alt- Ethnic } \\
\text { Identity }\end{array}$ & 23.93 & 8 & 2.992 & .002 & .872 & .759 & .032 & .124 & .069 & .183 & 49.933 \\
\hline
\end{tabular}




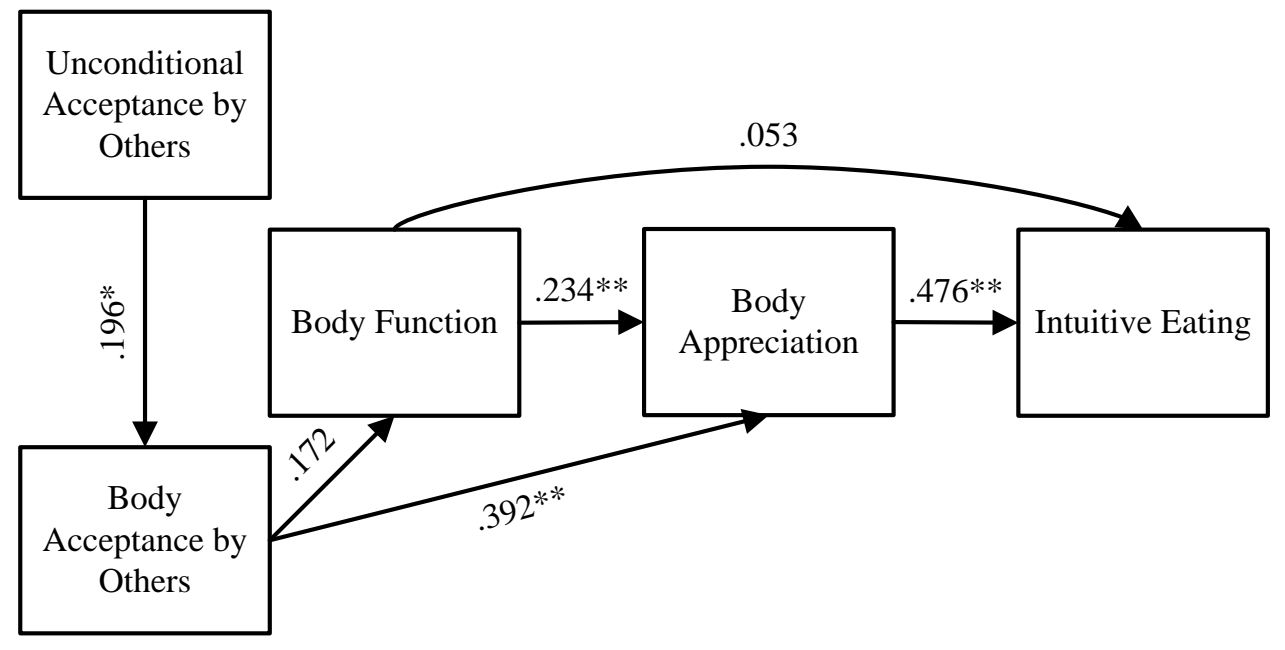

Figure 3. Original model of intuitive eating with path coefficients depicted from data obtained from 130 African American college women. *Correlation is significant at the 0.05 level. $* *$ Correlation is significant at the 0.01 level.

Approximately $3.10 \%$ of the variance in perceived body acceptance by others was explained by perceived unconditional acceptance by others, and $2.40 \%$ of the variance in body function was explained by perceived body acceptance by others. In addition, $35.9 \%$ of the variance in body appreciation was explained by perceived body acceptance by others and body function, and $32.5 \%$ of variance in intuitive eating was explained by body appreciation and body function.

In sum, the data failed to support Avalos and Tylka's model of intuitive eating with this sample, and thereby failed to provide empirical evidence for the generalizability of their model (Avalos \& Tylka, 2006). Nevertheless, the pathways between four 
variables were significant. These four significant pathways provide some evidence for relationships among variables within the model and thus offer support for previous research that suggested a relationship between some, but not all, variables within the model of intuitive eating among a small sample of African American college women (Avalos \& Tylka, 2006).

\section{Culturally Relevant Alternative Models With Outlier Cases Included in Data Analysis}

Alternative models in which general acceptance and ethnic/racial identity influence perceived body acceptance by others, and in which perceived body acceptance by others influences body function and body appreciation, body function influences body appreciation and intuitive eating, and body appreciation influences intuitive eating were examined next. First racial identity was included and then ethnic identity was included.

The alternative model with racial identity provided a poor fit to the data obtained from this sample of 130 African American college women (see Table 4). The chisquared statistic was significant, the chi-squared/df ratio was 6.362, and the CFI (.716), TLI (.468), SRMR (.093), and RMSEA (.204) all indicated a poor fit to the model. The poor fit failed to support Hypothesis 3 (i.e., that the culturally relevant alternative model provides adequate fit to the data obtained from sample of African American college women). Although the model provided a poor fit to the data, four paths (i.e., paths from general unconditional acceptance to perceptions of body acceptance by others, perceptions of body acceptance by others to body appreciation, body function to body 
appreciation, and body appreciation to intuitive eating) were significant at .05 level and provided support for hypotheses $4 \mathrm{~b}$ (i.e., general unconditional acceptance directly influences perceptions of body acceptance by others), $4 \mathrm{~d}$ (i.e., perceptions of body acceptance by others directly influences body appreciation), 4 f (i.e., body function directly influences body appreciation), and $4 \mathrm{~g}$ (i.e., body appreciation directly influences intuitive eating). Paths from racial identity to body acceptance by others, body acceptance by others to body function, and body function to intuitive eating were not significant and failed to support hypotheses $4 \mathrm{a}$ (i.e., ethnic/racial identity directly influences perception of body acceptance by others), $4 \mathrm{c}$ (i.e., perceptions of body acceptance by others directly influence body function), and 4e (i.e., body function directly influences intuitive eating). As four of the seven proposed paths were significant at .05, partial support for Hypothesis 4 was garnered. Path coefficients for the culturally relevant alternative model with racial identity are presented in Figure 4. 


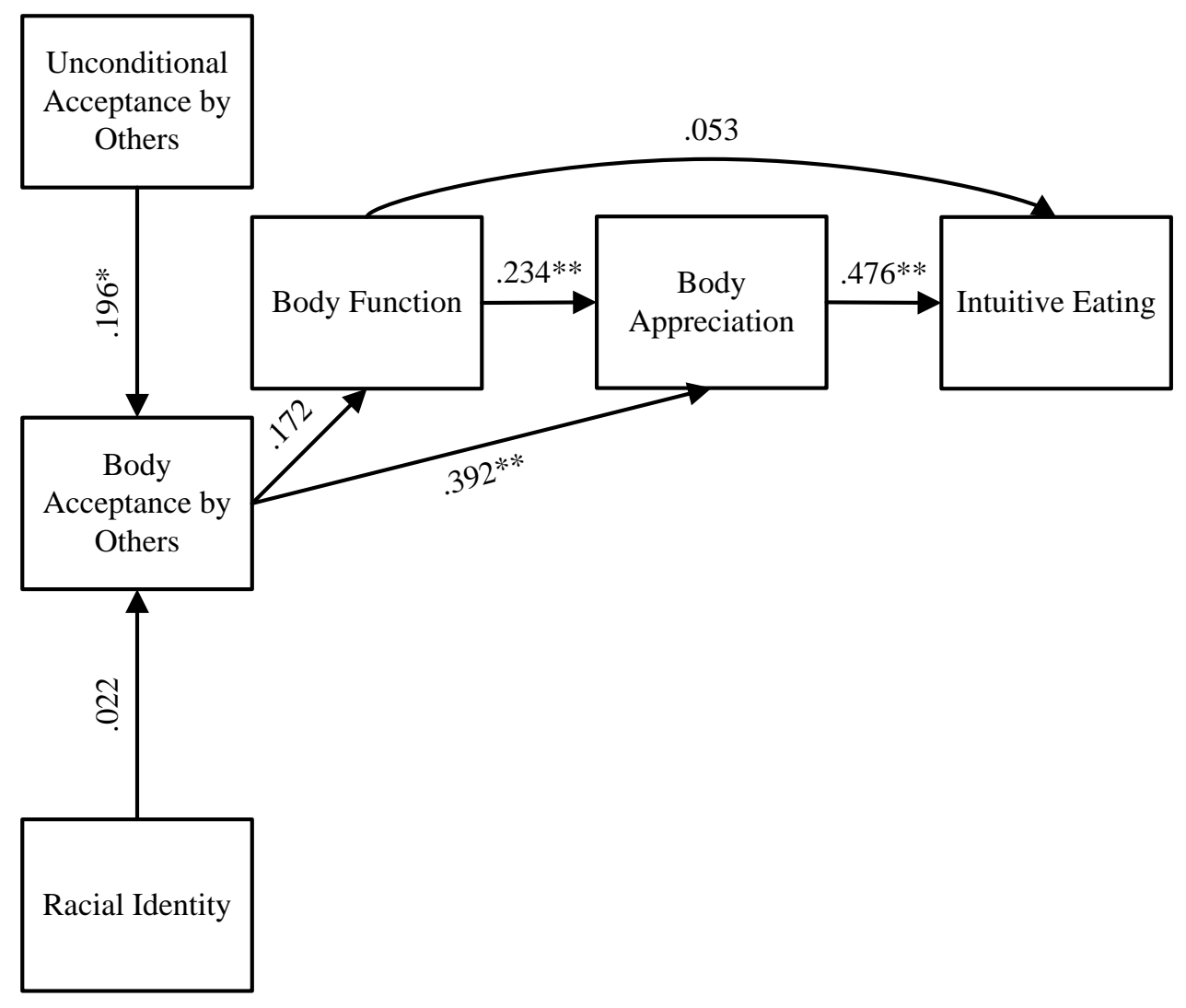

Figure 4. Culturally relevant alternative model of intuitive eating with racial identity and path coefficients depicted from data obtained from 130 African American college women. *Correlation is significant at the 0.05 level. **Correlation is significant at the 0.01 level.

Approximately $3.70 \%$ of the variance in perceived body acceptance by others was explained by perceived unconditional acceptance by others, and $2.40 \%$ of the variance in body function was explained by perceived body acceptance by others. In addition, $36.0 \%$ of the variance in body appreciation was explained by perceived body acceptance by 
others and body function, and $32.50 \%$ of variance in intuitive eating was explained by body appreciation and body function.

The culturally relevant alternative model with ethnic identity included instead of racial identity appeared to provide a slightly better fit to the data, however it, too, failed to support Hypothesis 3. As seen in Table 4, the chi-squared statistic was significant, the chi-squared/df ratio was 2.992, and the CFI (.872), TLI (.759), SRMR (.032), and RMSEA (.124) indicated only adequate to poor fit to the model. Paths from general unconditional acceptance by others to perceptions of body acceptance by others, body acceptance by others to body appreciation, body function to body appreciation, and body appreciation to intuitive eating again were significant and provided support for hypotheses 4b, 4d, 4f, and 4g. Paths from ethnic identity to body acceptance by others, body acceptance by others to body function, and body function to intuitive eating were not significant and again failed to provide support for hypotheses $4 \mathrm{a}, 4 \mathrm{c}$, and $4 \mathrm{e}$. As some paths in this culturally relevant alternative model were significant, Hypothesis 4 was partially supported. Path coefficients for the culturally relevant alternative model with ethnic identity are presented in Figure 5. 


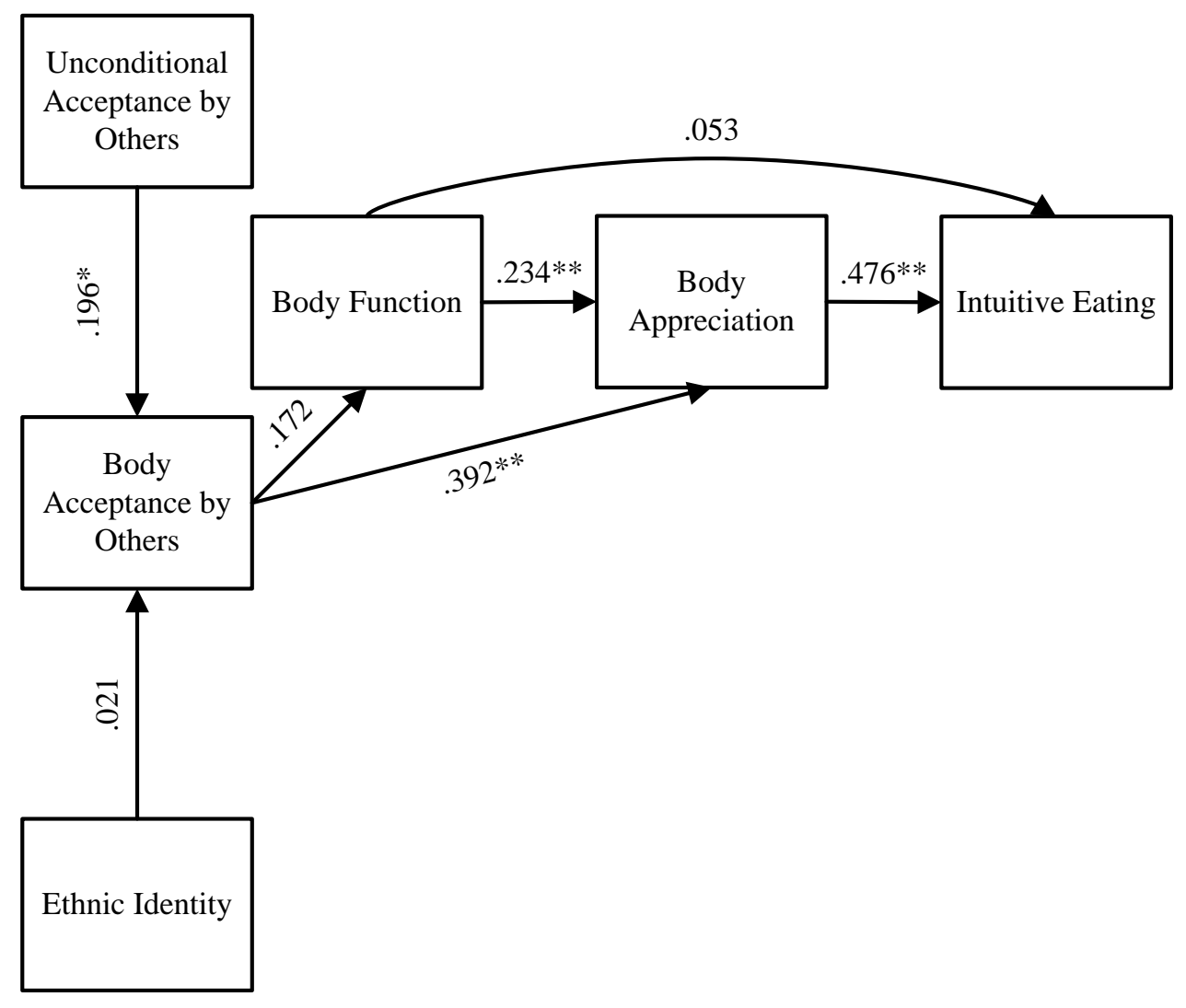

Figure 5. Culturally relevant alternative model of intuitive eating with ethnic identity and path coefficients depicted from data obtained from 130 African American college women. *Correlation is significant at the 0.05 level. **Correlation is significant at the 0.01 level.

Approximately $3.10 \%$ of the variance in perceived body acceptance by others was explained by perceived unconditional acceptance by others, and $2.40 \%$ of the variance in body function was explained by perceived body acceptance by others. In addition, 35.9\% of the variance in body appreciation was explained by perceived body acceptance by 
others and body function, and $32.5 \%$ of variance in intuitive eating was explained by body appreciation and body function.

\section{Comparison Among Models}

The original model of intuitive eating provided an adequate to poor fit to the data obtained from a sample of African American college women and neither of the two culturally relevant alternative models of intuitive eating provided a better fit to the data obtained from this sample of African American college women. As the original model provided an adequate to poor fit to the data and the data from the second culturally relevant alternative model provided an adequate to poor fit to the data, the Akaike's Information Criterion (AIC) was used to select the better fitting model. The AIC index is often used to make comparisons across competing models (Akaike, 1974) and the model with the lowest AIC index is often selected as the best model. The AIC indexes of the three models (i.e., original model, culturally relevant model with racial identity, and culturally relevant model with ethnic identity) are presented in Table 4. The original model provided the best fit of the three to the data, and as such the present findings failed to support Hypothesis 5 (i.e., the culturally relevant alternative models provide a better fit to the data than the original model). 


\section{Indirect Effects and Mediation}

Finally, bootstrapping (i.e., a computer-intensive procedure that samples from the observed data set to create an approximating distribution) procedures were used to examine indirect effects and mediation (Shrout \& Bolgers, 2002) within the original and culturally relevant alternative models of intuitive eating. Specifically, the researcher used an SPSS syntax file, which was developed by Preacher and Hayes (2004), to generate an approximating distribution to test the extent to which perceived body acceptance by others has an indirect influence on intuitive eating via its influence on body function and body appreciation in both the original and culturally relevant alternative models, and the extent to which culturally relevant variables (i.e., racial and ethnic identity) have an indirect influence on body function and body appreciation through body acceptance by others.

In order to test the indirect influence of perceived body acceptance by others on intuitive eating through body function and body appreciation, 1,000 bootstrap samples were created by randomly sampling the data from the 130 African American college women with replacements. Using the bootstrap sample, 1,000 estimates were generated with regard to the path from perceived body acceptance from others to body function and body function to intuitive eating. The 1,000 path estimates were multiplied together to generate a mean indirect effect with a $95 \%$ confidence interval. The mean indirect effect was .026 and the standard error of the mean effect was .022. Moreover, the $95 \%$

confidence interval for the mean indirect effect was -.005 (lower) and .080 (upper). As 
this $95 \%$ confidence interval includes zero, it was concluded that the indirect effect of body acceptance from others to intuitive eating through body function was not statistically significant at the .05 level (Shrout \& Bolger, 2002).

The researcher next utilized the above-noted procedure to test the indirect influence of perceived body acceptance by others on intuitive eating through body appreciation. The mean indirect effect was .180, the standard error of the mean effect was .042, and the $95 \%$ confidence interval was .102 (lower) and .272 (upper). As the 95\% confidence interval for the mean indirect effect did not include zero, it was concluded that the indirect effect of body acceptance from others to intuitive eating through body appreciation is statistically significant. Results therefore indicate that body acceptance by others indirectly influences intuitive eating through body appreciation but not through body function; this indicates partial support for Hypothesis $2 \mathrm{~g}$ (i.e., body acceptance by others indirectly influences intuitive eating through body function and body appreciation in the original model of intuitive eating) and 4i (i.e., body acceptance by others indirectly influences intuitive eating through body function and body appreciation in the culturally relevant alternative models).

In order to examine the influence of culturally relevant variables (i.e., racial and ethnic identity) on body function and body appreciation through body acceptance by others, the above noted procedures were utilized with racial and ethnic identity. First, one thousand path estimates were generated from racial identity to body acceptance by others and body acceptance by others to body function and body appreciation. The mean 
indirect effect from racial identity to body function was -.005 , the standard error of the mean effect was .010, and the $95 \%$ confidence interval was -.032 (lower) and .013 (upper). The mean indirect effect from racial identity to body appreciation was -.014 , the standard error of the mean effect was .022, and the $95 \%$ confidence interval was -.061 (lower) and .025 (upper). As the 95\% confidence interval for the mean indirect effects did include zero, it was concluded that the indirect effect of racial identity to body function and body appreciation through body acceptance by others is not statistically significant.

The mean indirect effect from ethnic identity to body function was calculated similarly and found to be .003 , the standard error of the mean effect was .031 , and the 95\% confidence interval was -.062 (lower) and .066 (upper). The mean indirect effect from ethnic identity to body appreciation was .009, the standard error of the mean effect was .069 , and the $95 \%$ confidence interval was -.128 (lower) and .144 (upper). Again, the $95 \%$ confidence intervals for these mean indirect effects included zero, thus it was concluded that the indirect effect of ethnic identity to body function and body appreciation through body acceptance by others is not statistically significant.

Culturally relevant variables (i.e., racial and ethnic identity), then, did not influence body function or body appreciation through body acceptance by others. Results do not support Hypothesis 4h (i.e., racial/ethnic identity indirectly influences body function and body appreciation through body acceptance by others). 


\section{Model Modification}

As the hypothesized models provided a relatively poor fit to the observed data, the research explored model respecifications (i.e., removing or adding paths) that could result in a better-fitting model. First, the researcher examined critical ratios of regression coefficients to identify estimated parameters that were not significantly different from zero and could be removed from the model. Critical ratios below 1.96 indicate that an estimated parameter is not significantly different from zero at .05 level and could be removed from the model (Tabachnick \& Fidell, 1992). In the present study, critical ratios associated with three parameters (i.e., racial/ethnic identity to body acceptance by others, body acceptance by others to body function, and body function to intuitive eating) were below standard cut-off score; this indicated that these parameters could be removed from model. When these three parameters (i.e., racial/ethnic identity to body acceptance by others, body acceptance by others to body function, and body function to intuitive eating) were removed, the model provided an adequate to poor fit to the data $\left(\chi^{2}=20.4, d f=\right.$ $\left.6.00, \chi^{2} / d f=3.41, p<.05, \mathrm{CFI}=.881, \mathrm{TLI}=.802, \mathrm{RMSR}=.049, \mathrm{RMSEA}=.137\right)$ and all remaining paths were significant at .05 level.

Second, the researcher calculated modification indices associated with set parameters to identify paths that could be added to improve model-data fit (Arbuckle, 2003). Modification indices around 100 indicate that set parameters are significantly different from zero and should be included within the model (Garson, 2009). In the present study, modification indices associated with set parameters were small (i.e., less 
than 25) and suggest that no set parameter was significantly different from zero and should be added to model.

\section{Summary}

Prior research (Swami \& Chomorro-Premuzic, 2008) suggested the possibility of cross-cultural differences in the factor-structure of the Body Appreciation Scale (Avalos et al., 2005) and therefore a factor analysis was conducted on data obtained from these 130 African American college women. Factor analysis suggested that multiple one and two-factor models provided an adequate model-data fit, however no model provided excellent model-data fit. Consequently, the most parsimonious model (i.e., the one-factor model with 13 items) was determined to provide the best fit to the data. As the one-factor model with 13 items provided the best fit to the data, support was garnered for the unidimensional factor structure of the Body Appreciation Scale among African American college women.

Path analysis was then used to determine whether the original model of intuitive eating forwarded by Avalos and Tylka (2005) provided a good fit to the data from these 130 African American women. That model of intuitive eating provided an adequate to poor fit to the data and only four of the six proposed pathways within the model were upheld among this sample of African American college women.

Next, culturally relevant variables (i.e., racial and ethnic identity) were added to the model as an attempt to improve the model-data fit for a sample of African American 
college women. Unexpectedly, the alternative model with ethnic identity provided an adequate to poor fit to the data, and the alternative model with racial identity provided a poor fit to the data. Only four of the seven proposed relationships within these models were upheld. It should be noted that the paths from the culturally relevant variables (i.e., ethnic and racial identity) were not significant.

Finally, Akaike's Information Criterion (AIC) was used to compare model fit across the three models tested (Akaike, 1974). Index values suggested that the original model offered the best, albeit limited, fit to the present data. Overall, results of this investigation provide modest support for selected hypotheses and for the generalizability of the body appreciation and intuitive eating research to African American college women. 


\section{CHAPTER V \\ DISCUSSION}

Previous research supported the model of intuitive eating (Avalos \& Tylka, 2006) and propositions within the model of intuitive eating with samples of White, middle to upper-middle class women (Augustus-Horvath, 2008; Avalos \& Tylka, 2006; Tylka, 2007). In addition, data from approximately 40 African American college women provided preliminary support for several relations within the model of intuitive eating (Avalos \& Tylka, 2006); thus, additional research seemed needed with a sufficient sample of African American college women to extend the model of intuitive eating and propositions within the model (Avalos \& Tylka, 2006). The present study adds to the extant literature on body image and eating behaviors by exploring a model of intuitive eating and relations within the model with a sample of 130 African American college women.

Moreover, the present study integrated culturally relevant variables (i.e., ethnic/ racial identity) into the model of intuitive eating and explored these culturally relevant alternative models with this sample of African American college women. The incorporation of culturally relevant variables into the model of intuitive eating answers 
the call of researchers both to integrate additional variables into the model of intuitive eating (Avalos \& Tylka, 2006; Tylka, 2007) and to consider the impact of cultural values, beliefs, and practices when exploring positive constructs among diverse samples (Constantine \& Sue, 2006).

\section{Comparisons of the Intuitive Eating Models}

All three of the models (i.e., original, culturally relevant alternative model with racial identity, and culturally relevant alternative model with ethnic identity) provided a relatively poor fit to the data obtained from 130 African American college women. These findings stand in contrast to the hypotheses of the current study and previous research that explored a model of intuitive eating with samples of young, mostly White college women (Avalos \& Tylka, 2006) and women of diverse ages (Augustus-Horvath, 2008). Previous research (Augustus-Horvath, 2008; Avalos \& Tylka, 2006) suggested that the model of intuitive eating provided an adequate to excellent fit to sample data, especially after the nonsignificant path from unconditional acceptance to body function was deleted.

The observed discrepancy in model-data fit between these prior studies and the present one may be attributed to the fact that the model of intuitive eating was developed and modified almost exclusively on the experience of young, middle to upper middle class, European American college women (Avalos \& Tylka, 2006). The experience of 
African American college women may not be fully captured by variables or associations among variables within the model, and model modification maybe warranted.

Indeed, modification indices suggested that all three models could provide a better, but still inadequate, fit to the observed data if the paths from culturally relevant variables to body acceptance by others, body acceptance to body function, and body function to intuitive eating were removed. Removing paths from body acceptance by others to body function and body function to intuitive eating, however, contradicts theoretical assertions that perceptions of body acceptance by others influence women's emphasis on internal experiences rather than external appearances (Avalos \& Tylka, 2006: Fredrickson \& Roberts, 1997) and that emphasis on internal experiences rather than external appearances influences intuitive eating (Avalos \& Tylka, 2006; Tribole \& Resch, 1995).

Although these suggested model modifications are inconsistent with theory, they support to some degree the post hoc analysis of Avalos and Tylka (2006) who found that body function is not significantly correlated with perceptions of body acceptance or intuitive eating among African American college women. In the present study, body acceptance was not correlated with body function; however, a small, but significant, correlation existed between body function and intuitive eating. When body function was considered in the overall models, the small correlation between body function and intuitive eating was rendered nonsignificant, and perceptions of body acceptance exerted 
an influence on intuitive eating solely through its association with body appreciation among African American college women.

Weak associations among the above-noted variables may be attributed to the validity of the measure used to assess body function (i.e., Body Surveillance subscale of the Objectified Body Consciousness Scale, McKinley \& Hyde, 1996). To date, no researcher has explored the psychometric properties of the Body Surveillance subscale with the alternative scoring procedure, which was utilized in this study, among samples of African American college women. Buchanan et al. (2008) utilized the Body Surveillance subscale with the standard scoring procedure to measure habitual body monitoring among 117 African American women. Buchanan et al. (2008) found moderate internal consistency reliability $(\alpha=.76)$ and preliminary evidence of criterionrelated validity (i.e., body surveillance correlated with skin-tone surveillance) for the subscale. Lower, albeit acceptable, levels of internal consistency reliability $(\alpha=.72)$ were found in this study. Additional research, however, is needed to explore the psychometric properties of the Body Surveillance subscale and Body Surveillance subscale with alternative scoring procedure among African American college women. Regardless, the finding that perceptions of body acceptance by others exerted an influence on intuitive eating solely through the association with body appreciation among African American college women represents a slight deviation from the original model of body appreciation and intuitive eating proposed by Avalos and Tylka (2006). This finding, nevertheless, seems consistent with previous research (Buchanan et al., 2008) 
that found objectification theory did not fully capture the experience of African American women and model modifications were warranted. Results of the present study, in conjunction with those of Buchanan et al. (2008), suggest both similarities and differences in the prediction of body image and eating behaviors among African American and European American women. This pattern also parallels the eating disorder literature that notes both similarities and differences in prevalence and severity of eating disorder symptomatology among African American and European American participants (e.g., O’Neill, 2003; Wildes, Emery, \& Simons, 2001).

Although the present models did not provide an excellent fit to the data and model modifications may be warranted, all three model versions accounted for a considerable portion of variance in intuitive eating and produced significant relations among several, but not all, variables within the model of body appreciation and intuitive eating. Variance accounted for in intuitive eating and significant relations among variables provide empirical support for some theoretical assertions underlying a model of intuitive eating and previous research.

Specifically, in the present study, variables within the three models explained approximately $33 \%$ of variance in intuitive eating. This percentage of variance in intuitive eating explained by variables within the three models was similar to the percentage of variance in intuitive eating explained (i.e., 35\%) when Avalos and Tylka (2006) utilized path analysis procedures to explore their model of intuitive eating with approximately 180 college women. Although similar to Avalos and Tylka's study that 
utilized path analysis procedures, the percentage of variance in intuitive eating explained by variables within the present three models was smaller than percentages explained by other studies (Augustus-Horvath, 2008; Avalos \& Tylka, 2006) that utilized latent variable structural equation modeling. Authors who used latent variable structural equation modeling to control for measurement error found that variables within their models explained approximately $43 \%$ of variance in intuitive eating for larger samples of young, mostly European American college women (Avalos \& Tylka, 2006) and women of diverse ages (Augustus-Horvath, 2008).

Unexpectedly, the integration of culturally relevant variables did not account for additional variance in intuitive eating. The discrepancy appears because of the weak associations between the culturally relevant variables (i.e., racial/ethnic identity) and the criterion variables. In the present study, culturally relevant variables were not significantly associated with perceptions of body acceptance by others, body function, or body appreciation. Weak associations between racial/ethnic identity and these criterion variables is somewhat consistent with the previous, albeit pathologically focused, research of Abrams et al. (1993), Akan and Grilo (1995), Baugh (2005), Gray (1993), and Peterson (2000) that indicated no significant relationship exists between racial/ethnic identity and body dissatisfaction or eating disorders.

Results of the current study also support the research findings of Harris (1995) that culturally relevant variables failed to influence African American women's body satisfaction when personal, social, or interpersonal variables were included. In the 
present study, other types of variables (i.e., contextual and intrapersonal) exerted a stronger influence on body appreciation and eating behavior than did the culturally relevant variables. Although contextual and intrapersonal variables exerted a stronger influence on body appreciation and eating behavior, these findings provide some preliminary information on the role culturally relevant variables play (or do not play) in models of body appreciation and eating behaviors.

The culturally relevant variables (i.e., racial/ethnic identity) also were negligibly related to one another in the present study. The negligible relationship among the culturally relevant variables may suggest that racial identity, as defined by scores on the Internalization Afrocentricity subscale of The Cross Racial Identity Scale (Vandiver et al., 2000), and ethnic identity, as defined by scores on the Ethnic Identity subscale of The Multigroup Ethnic Identity Measure (Phinney, 1992) assess different latent constructs, and neither of them are significantly associated with the variables within the model.

Instead of high levels of identification with African American culture, evident by scores on the Afrocentricity subscale of The Cross Racial Identity Scale (Vandiver et al., 2000) or the Ethnic Identity subscale of The Multigroup Ethnic Identity Measure (Phinney, 1992), it may be that low levels of identification with African American culture (e.g., CRIS assimilation, miseducation, \& self-hatred subcales) are associated negatively with body appreciation and intuitive eating. Previous research supports an association between low levels of identification with African American culture and body dissatisfaction and disordered eating (Abrams et al., 1993). Future research, then, should 
explore the relationship between identification with African American culture at all levels and body appreciation/intuitive eating.

\section{Specific Relations Among Variables}

Across models tested, unconditional acceptance exerted a small, albeit significant, influence on body acceptance. Both body acceptance and body function exerted a moderate influence on body appreciation, but body acceptance exerted a stronger influence on body appreciation than did body function. In fact, body acceptance exerted an indirect influence intuitive eating through its association with body appreciation. Finally, body appreciation exerted a strong influence on intuitive eating.

The above-noted relations among variables parallel the post hoc finding of Avalos and Tylka (2006). Using data from 38 African American college women, Avalos and Tylka (2006) found significant correlations between general unconditional acceptance and body acceptance, body acceptance and body appreciation, body function and body appreciation, body appreciation and intuitive eating. Correlations between body acceptance and body function and body function and intuitive eating, however, were not significant. Taken together, the relations among variables illustrated in Avalos and Tylka's work and the present research provide empirical support for the application of a selected set of the theoretical propositions underlying the model of intuitive eating to African American college women. 
Specifically, this study's findings support the theoretical propositions of Rogers (1961) that general unconditional acceptance promotes perceptions of acceptance in particular areas of self (e.g., body acceptance). In addition, the present results are consistent with the propositions of Fredrickson and Roberts (1997) and Avalos and Tylka (2006) that environmental conditions may influence eating behaviors through one's satisfaction with body image. Specifically, Fredrickson and Roberts (1997) suggested that negative environmental conditions (e.g., pressure for thinness) influence eating disorder symptomatology through body shame and body dissatisfaction; Avalos and Tylka (2006) posited that positive environment conditions (e.g., acceptance regarding body size and shape) influence adaptive eating through appreciation and respect toward the body. Fredrickson and Roberts and Avalos and Tylka further suggested that surveillance of internal experiences and external appearance predicts satisfaction with body image, and this relation was supported in the present models. Women's focus on internal experiences rather than external appearance predicted appreciation of their body. Findings also supported the assertions of Tribole and Resch (1995), Avalos et al. (2005), and Tylka (2006) that appreciation and respect toward the body regardless of congruence with standards or beauty or thin-ideal predicts eating the type and amount of food desired in response to internal signals of hunger and satiety. Finally, results supported assertions of Avalos and Tylka (2006) that at least some of the relations within the body appreciation and intuitive eating model extend to fit the experience of African American college women. 
In addition to garnering support for some theoretical propositions related to the model of intuitive eating, findings from the present study were consistent with empirical research exploring adaptive and maladaptive eating among women. Relations in the present data are consistent with empirical research of Augustus-Horvath (2008), Buchanan et al. (2007), Moradi and Rottenstein (2007), Avalos and Tylka (2006), Tylka and Hill (2004), Thomas (2001), and Fredrickson and Roberts (1997) that societal messages influences body image satisfaction among a broad range of women, and with the work of Augustus-Horvath (2008), Avalos and Tylka, Tylka and Hill (2004), Moradi and Rottenstein, Noll and Fredrickson (1998), Fredrickson and Roberts, and Cattarin and Thompson (1994) that body image satisfaction is related to eating behaviors. The present results are also consistent with research of Tylka and Subich (2004) and Muehlenkamp and Saris-Baglama (2002) that established an association between attention to internal experiences and body image satisfaction. Women, who focused on internal experiences rather than external appearance, have higher levels of body satisfaction. Finally, the results of this study supported findings of Augustus-Horvath (2008) that several pathways within the model of the intuitive eating can be extended to more diverse samples of women (e.g., women of diverse ages and African American college women).

\section{African American Women's Eating Attitudes}

Relations among variables provided empirical support for several theoretical propositions underlying models of body appreciation and intuitive eating among African 
American college women. It seems equally important, however, to explore the present data in the context of past research because so little of that prior work included diverse samples of women.

An inspection of the means for the constructs of interest in the present research revealed that African American college women reported slightly higher levels of body acceptance, body appreciation, and intuitive eating than those reported for young, mostly European American, college women (Avalos \& Tylka, 2006) or women of diverse ages (Augustus-Horvath, 2008) in other published studies. Higher levels of body acceptance, body appreciation, and intuitive eating among African American women lend support to the similar, albeit pathologically focused, findings of Cashel et al. (2003) and Wildes et al. (2001) that African American women reported lower levels of body dissatisfaction, desire to lose weight, and dietary restraint than European American counterparts. In addition, findings support the assertions of Choate (2005), Harris (1994), and Molloy and Herzberger (1998) that certain factors in the African American community may promote a positive body image and protect some African American women from disordered eating.

Although African American college women had slightly higher levels of body appreciation and intuitive eating, the strength of association between these variables paralleled prior studies. Across all samples, body appreciation exerted a strong influence on intuitive eating, suggesting this conceptual relation is relevant across groups of women. 
Both body acceptance and body function exerted a moderate influence on body appreciation. However, the moderate influence was less substantial than associations reported in previous research among samples of young, mostly European American college women (Avalos \& Tylka, 2006) and women of diverse ages (Augustus-Horvath, 2008), and suggests that body acceptance and body function are less powerful predictors of body appreciation among African American college women than other groups of women.

Unconditional acceptance, which was measured by a score on 36-item version of Barrettt-Lennard Relationship Inventory (Barrett-Lennard, 1962; Claiborn et al., 1983) exerted a small, albeit significant, influence on body acceptance by others. The small influence of unconditional acceptance on body acceptance by others also parallels data from prior research with samples of young, mostly European American college women (Avalos \& Tylka, 2006) and suggests that unconditional acceptance did not exert a strong influence on other variables within the model through its association with body acceptance. Weak association between unconditional acceptance by others and body acceptance contrasts with the findings of Augustus-Horvath (2008). Augustus-Horvath (2008), however, used the Social Provision Scale (Russell \& Cutrona, 1984; Cutrona \& Russell, 1987) to measure unconditional acceptance among women of diverse ages. Using the Social Provision Scale, they found a strong association between unconditional acceptance and body acceptance by others. The utilization of different instruments to measure unconditional acceptance makes direct comparisons difficult. 
Results of the present data in relation to past findings suggest the possibility of similarities and differences in the prediction of body appreciation and intuitive eating among groups of women. This possibility supports the finding of Augustus-Horvath (2008) that variables within the model of body appreciation and intuitive eating are associated at slightly different strengths among women aged 18-24 and 25-79. These findings also seem consistent with previous, albeit pathologically focused, research (Buchanan et al., 2008; O'Neill, 2003; Wildes et al., 2001) that reported both similarities and differences in the prediction of body image and eating behavior among groups of women (e.g., European American college women and African American college women).

\section{Factor Structure of Body Appreciation Scale}

Given the dearth of research on the Body Appreciation Scale with African American college women and possibility of cross-cultural difference (Swami \& Chamorro-Premuzic, 2008), the present study also explored the psychometric properties (i.e., factor structure and internal consistency reliability) of the Body Appreciation Scale with these African American college women and determined that the Body Appreciation Scale seems a valid measure of body appreciation, although questions remain about its factor structure.

A one-factor model with all 13 items on the Body Appreciation Scale serving as indicator for the body appreciation latent variable provided an adequate fit to the present data obtained from 130 African American college women and supported the 
unidimensional factor structure of the Body Appreciation Scale that had been found mostly among European American college women (Avalos et al., 2005), Austrian men and women (Swami, Stieger, Haubner, \& Voracek, 2008), and British men and women (Swami, Hadji-Michael, \& Furnham, 2008). Although the one-factor model provided an adequate fit to the data, three items (i.e., items $8,9,12$ ) had weak or nonsignificant loadings on latent variable body appreciation. Moreover, the fit of the one-factor model improved when error terms associated with these three items correlated. This improved fit suggests that the covariance among three items may be related to the latent variable of body appreciation and at least one other unknown common source.

That source of unknown common variance may be related to body image investment. With that in mind, a two-factor model also was explored in which the three items noted previously served as indicators for a latent body image investment variable and other items served as indicators for the body appreciation latent variable. Results of this analysis suggested that this two-factor model provided an adequate fit to the data and was consistent with the two-factor structure of the Body Appreciation Scale found for a sample of Malaysian women (Swami \& Chamorro-Premuzic, 2008).

The internal consistency reliability observed $(\alpha=.87)$ in conjunction with the factor analytic findings provide preliminary support for the psychometric soundness of the Body Appreciation Scale among African American college women. The Body Appreciation Scale seems a relatively sound measure of body appreciation among African American college women, and all 13 items can be used to calculate a total body 
appreciation score. However, additional research should be conducted to clarify the factor structure and examine other psychometric properties (e.g., validity, test-retest reliability) of the Body Appreciation Scale with African American college women.

\section{Limitations}

Although the present study contributes to extant literature on body image and eating behaviors, it contains limitations that are important to address. First, the researcher approached African American women at a large Midwestern university and offered the women and their friends an opportunity to participate in research on African American women's eating behaviors in exchange for a small, cash award (\$5.00). This limited the sample to those women present at a specific time and place. The responses from those African American college women may or may not be representative of other African American college women. Thus, the present research may not generalize beyond this small, nonrepresentative sample.

Second, all of the data were collected at the same point of time so causal conclusions about the relations of variables within the models cannot be drawn. Consequently, there exists the possibility that a different sequence of variables could fit the data from these African American college women equally well or better.

A third limitation to the present study is the exclusive use of self-report measures. Self-report measures rely on an individual's ability to accurately recognize and report internal experiences (e.g., thoughts, feelings, general experiences). Inability to recognize 
internal experience (i.e., low interoceptive awareness) and social desirability (i.e., impression management; self-deceptive enhancement) may introduce error into the data and results from data analysis. Unfortunately, in the present research, no assessment of social desirability was gathered.

A fourth limitation to the present study is the limited assessment of unconditional acceptance by others. Participants were asked to consider important/ influential persons (e.g., Mother, Father, Grandmother, Grandfather, Older Brother, Older Sister, Uncle, Aunt, Older Cousin, Teacher, Coach, Employer/Supervisor, Counselor, \& Clergy) in their lives when they were growing up. Asking participants to consider collectively the important/ influential persons in their lives is more inclusive than the previous research that assessed unconditional acceptance from the single most important person when they were growing up (Avalos \& Tylka, 2006). Although more inclusive than previous research, assessing perceptions of unconditional acceptance from important/ influential persons fails to capture overall levels of acceptance within their environment (Avalos \& Tylka, 2006). For example, a woman may receive messages from her immediate family that others accept her and do not wish for her to modify internal or external appearance; however, she may also receive messages from peers and the media that she should alter her internal and external appearance to be accepted by peers and society. In this example, assessing messages exclusively from important others (e.g., family) fails to capture overall levels of acceptance within the environment. 
In addition, perceptions of unconditional acceptance by others may change over time. For example, a woman may have received overt messages from family when she was growing up that others accept her unconditionally; however, as an adult she may receive messages from romantic partners, peers, and society that she needs to alter internal or external appearance to fit societal standards. Current levels of unconditional acceptance from a variety of sources (e.g., romantic partners, peers, society) may have a greater impact on current intuitive eating than do perceived levels of unconditional acceptance from important others in an earlier developmental period (Avalos \& Tylka, 2006).

Still another limitation to the current study is the lack of definitive support for the psychometric properties of the Barrett-Lennard Relationship Inventory (Barrett-Lennard, 1962; Claiborn et al., 1983), Body Acceptance by Others Scale (Avalos \& Tylka, 2006), Body Surveillance subscale of the Objectified Body Consciousness Scale (McKinley \& Hyde, 1996), Body Appreciation Scale (Avalos et al., 2005) and Intuitive Eating Scale (Tylka, 2006) when used among African American college women. In fact, a visual inspection of items on the Body Surveillance subscale of the Objectified Body Consciousness Scale (McKinley \& Hyde, 1996) illuminated a potential threat to content validity of the scale among African American college women. One-quarter of the items on the subscale assess whether the clothes participants select make them look good. As fashion is extremely important in the African American community (Choate, 2005), a substantial number of items on the Body Surveillance subscale may be assessing how 
African American women feel their body appears in certain clothes rather than how it appears overall (e.g., clothes, body size, body shape) to others. To date research had not established the validity or reliability of the Body Surveillance subscale, or indeed the other measures highlighted above, among African American college women. The current study provides some preliminary evidence for the factor structure of the Body Appreciation Scale and for the acceptable internal consistency (i.e., Cronbach's alpha) of the all scales; however, future researchers should explore further the psychometric properties of these measures with samples of African American college women.

In the present study, too, African American women's body mass index was not assessed. Previous research established a negative association between body mass index and intuitive eating (Hawks et al., 2005; Tylka, 2006) and explored a model of intuitive eating with body mass index controlled as a covariate (Augustus-Horvath, 2008). Failure to assess or control for body mass index may have affected the results of the present study, and future research should account for impact of women's body mass index on variables within the model of intuitive eating (Avalos \& Tylka, 2006).

Finally, the researcher conducted multiple path analyses with the data from 130 African American college women and treated each analysis as though it was independent. Utilizing the same data to conduct multiple analyses may have increased the probability of finding a relationship among variables by chance alone, even if no real relationship exists within the data (Tabachnick \& Fidell, 1992). Thus, replication of these findings seems essential. 


\section{Implications for Practice and Research}

Notwithstanding the above-noted limitations, results from the present study have implications for practitioners promoting positive body image and adaptive eating practices among African American college women and for researchers exploring similarities and differences in the prevalence and predictors of body image and eating behaviors among African American and European American college women. More specifically, results from the present study can be used to help practitioners promote positive body image and adaptive eating practices among African American college women. The present findings highlight the relationship between body appreciation and intuitive eating. Among African American college woman, body appreciation may be an important construct in translating perceived body acceptance from others and body function into eating behaviors Therefore, practitioners should consider focusing their efforts on promoting a positive body image in African American women.

In order to promote positive body image in African American women, practitioners may need to explore messages from a variety of sources (e.g., family, friends, romantic partners, society). Clinicians should work with clients to identify and challenge messages that clients need to change their physical appearance by losing weight (Avalos \& Tylka, 2006). In addition, clinicians may consider outreach programming that highlights for all university students the importance of communicating body acceptance to friends and peers. 
Also, clinicians can work with African American college women to attend more closely to their internal experiences (e.g., thoughts, feeling, internal signals of hunger and satiety). Specifically, clinicians may utilize mindfulness-based interventions to help clients increase attention to internal experiences. Findings from the current study suggest that attention to internal experiences may be associated with body appreciation and body appreciation may be associated with intuitive eating.

Results from the present study extend research that explored difference in the prevalence and severity of body image and eating behaviors among African American and European American woman. The results of this study extend the previous, albeit more pathological literature, by suggesting that both similarities and differences exist in the promotion and maintenance of positive body image and adaptive eating behavior among African American and European American college women. The presence of similarities and differences in the promotion of positive body image and eating behaviors in African American and European American college students suggests that clinicians should be cautiously optimistic in generalizing the findings of this body of literature, much of which is based on samples of European American college women, to African American clients. Moreover, they suggest that future researchers should explore other variables that may be relevant to cross-cultural differences in the model of intuitive eating.

Although the sample of African American college women included in the present study was larger than samples used in previous research, it was still relatively small 
(Tabachnick \& Fidell, 2001). Thus, it seems wise to replicate this study with a larger sample of African American college women. If subsequent research continues to suggest nonsignificant paths from general unconditional acceptance to body acceptance, racial/ethnic identity to body acceptance, body acceptance to body function, or body function to intuitive eating, exploration of modified models may be in order.

Future researchers, also, should consider the impact of body mass index on variables within the models. Avalos and Tylka (2006) noted that women with different body mass indices may receive different messages from important others and society about their need to lose weight. Specifically, Avalos and Tylka (2006) speculated that women with higher body mass indices may receive frequent and direct messages from important others and society that they need to lose whereas women with lower body mass indices may receive messages from important others and society that look good and do not need to lose weight. And, different messages that women may have received based on their body mass indices might exert an unintentional influence on variables within the model. In fact, Augustus-Horvath (2008) found that body mass index exerted an unintentional influence on variables within the model such that the relationship between social support and intuitive eating was significant. When body mass index was controlled as a covariate, the relationship between social support and intuitive eating was rendered nonsignficant. Given the suggestion of Avalos and Tylka (2006) and the influence of body mass index seen in previous research (Augustus-Horvath, 2008), future 
researchers should measure body mass index and explore relationships among variables with body mass index controlled as a covariate.

In addition, future researchers should consider exploring models of intuitive eating with another statistical procedure. Structural equation modeling offers many advantages over path analysis that may prove beneficial to the exploration of intuitive eating (Weston \& Gore, 2006). Specifically, structural equation modeling allows the researcher to utilize multiple indicators to represent constructs of interest and explore the relationships among constructs, not indicators, within the models (Weston \& Gore, 2006). In lieu of exploring two culturally relevant alternative models of intuitive eating, with SEM a researcher could utilize other aspects of identity to operationalize African American culture and directly explore the relationship between African American culture and other constructs within the model of intuitive eating.

Some researchers (Augustus-Horvath, 2008; Avalos \& Tylka, 2006) who explored the model of intuitive eating have utilized structural equation modeling; however, they elected to use item parcels instead of multiple indicators of constructs. Although the item-parceling strategy circumvents some practical concerns (e.g., administrations of additional measures), it fails to capitalize on the strength of structural equation modeling. Although limited measures of variables within the model of intuitive eating exist, researchers could develop or borrow measures from other health care disciplines that assess positive constructs. For example, future researchers could utilize 
the 21-item Intuitive Eating Scale (Tylka, 2006) and 27-item Intuitive Eating Scale (Hawks et al., 2004) to assess the intuitive eating construct.

As future researchers explore models of intuitive eating, it will be imperative to consider the role of additional variables within the model (Avalos \& Tylka, 2006). Exploring an abbreviated model of intuitive eating, Tylka (2007) found that proactive coping and self-esteem strengthened the association between perceived body acceptance from others and intuitive eating and accounted for additional variance in intuitive eating. As proactive coping and self-esteem accounted for additional variance in intuitive eating, future researchers could incorporate proactive coping and self-esteem into the model of intuitive eating and explore the expanded model of intuitive eating with a large sample of African American and European American college women.

Similarly, future researchers could integrate various health indicators within the model and explore the association between intuitive eating and health among samples of African American and European American college women. Hawks et al. (2005) found an association between intuitive eating and various health indicators (e.g., triglycerides, high-density lipoproteins, low-density lipoproteins, cardiovascular health, body mass index) among a sample of young, healthy, college students. As Hawks et al. (2005) identified an association between intuitive eating and various health indicators, future researcher could incorporate various health care indicators into the model of intuitive eating and explore the expanded model with samples of college women. 
Exploring models of intuitive eating simultaneously with samples of both African American and European American college women would allow researchers to determine whether constructs within the model of (e.g., unconditional acceptance, body acceptance, body function, body appreciation, intuitive eating) differ among subsamples. Moreover, exploring models with African and European American subsamples would allow researchers to compare adequacy of fit directly and explore model invariance using multiple group analysis procedures. These explorations and analyses would help researchers determine whether path estimates differ between African and European American college women.

In addition to integrating additional constructs and exploring relationships among constructs within the model of intuitive eating among both African American and European American college women, future researchers could contribute to the extant literature by evaluating psychometric properties of measures within the model. Tylka (2006) developed and psychometrically evaluated the Intuitive Eating Scale with samples of young, mostly European American, middle to upper-middle class, college women; thus additional research is needed to determine whether the psychometric properties of the Intuitive Eating Scale are upheld among samples of African American college women and other samples (e.g., men, community samples, sexual minorities, athletes). Findings from this study garnered preliminary evidence for the Intuitive Eating Scale's internal consistency among a sample of 130 African American college women. However, additional research is needed to examine other psychometric properties (e.g., factor 
structure, temporal stability, construct validity) among African American college women and other groups.

Future researchers also should attempt to identify causal relationships via longitudinal or qualitative studies. Identifying the variable (or set of variables) that precede another variable (of set of variables) in sequence could advance the body appreciation and intuitive eating literature.

Finally, qualitative studies which explore African American college women's experience of intuitive eating and the factors impacting intuitive eating may help to move research closer toward a complete picture of body image and eating behaviors among African American women. Studies that assess intrapersonal, interpersonal, and sociocultural influences on intuitive eating may present a limited view of reality. Other factors (i.e., access to food or financial resources to purchase food) may influence intuitive eating. Perhaps, qualitative studies will illuminate the myriad of other factors impacting intuitive eating among African American college women. 


\section{REFERENCES}

Abood, D. A., \& Chandler, S. B. (1997). Race and the role of weight, weight change, and body dissatisfaction in eating disorders. American Journal of Health Behavior, $21,21-25$.

Abrams, K. K., Allen, L. R., \& Gray, J. J. (1993). Disordered eating attitudes and behavior, psychological adjustment, and ethnic identity: A comparison of black and white female college students. International Journal of Eating Disorders, 14, 49-57.

Adams, G. R., Bennion, L., \& Huh, K. (1987). Objective measure of ego identity status: A reference manual. Unpublished manuscript, Utah State University, Logan, UT.

Akaike, H. (1974). A new look at the statistical model identification. Automatic Control, $19,716-723$.

Akan, G. E., \& Grilo, C. M. (1995). Sociocultural influences on eating attitudes and behaviors, body image, and psychological functioning: A comparison of AfricanAmerican, Asian-American, and Caucasian college women. International Journal of Eating Disorders, 18, 181-187.

Allan, J. D., Mayo, K., \& Michel, Y. (1993). Body size values of white and black women. Research in Nursing \& Health, 16, 323-333.

Arbuckle, J. L. (2003). AMOS 5.0 update to the AMOS user's guide. Chicago, IL: SmallWaters Corp.

Arnow, B., Kenardy, J., \& Agras, W. S. (1995). The Emotional Eating Scale: The development of a measure to assess coping with negative affect by eating. International Journal of Eating Disorders, 18, 79-90.

Aruguete, M. S., Nickleberry, L. D., \& Yates, A. (2004). Acculturation, body image, and eating attitudes among black and white college students. North American Journal of Psychology, 6, 393-404. 
Atkinson, D. R., Morten, G., \& Sue, D. W. (1983). Counseling American minorities:A cross-cultural perspective. Dubuque, IA: Brown.

Atkinson, D. R., Morten, G., \& Sue, D. W. (Eds.). (1998). Counseling American minorities: A cross cultural perspective (6th ed.). New York: McGraw Hill Publishers.

Augustus-Horvath, C. L. (2008). A test and extension of an acceptance model of intuitive eating with younger and older women. Unpublished doctoral dissertation, The Ohio State University.

Avalos, L., \& Tylka, T. L. (2006). Exploring a model of intuitive eating with college women. Journal of Counseling Psychology, 53, 486-497.

Avalos, L., Tylka, T. L., \& Wood-Barcalow, N. (2005). The Body Appreciation Scale: Development and psychometric evaluation. Body Image, 2, 285-297.

Bacon, L., Kleim, N. L., Van Loan, M. D., Derricote, M., Gale, B., Kazaks, A., et al. (2002). Evaluating a "non-diet" wellness intervention for improvement of metabolic fitness, psychological well-being and eating and activity behaviors. International Journal of Obesity, 26, 854-865.

Bacon, L., VanLoan, M. D., Stern, J. S., \& Keim, N. L. (2005). Size acceptance and intuitive eating improve health for obese female chronic dieters. Journal of American Dietetic Association, 105, 929-936.

Baldwin, J. A., \& Bell, Y. R. (1982). The African Self-Consciousness Scale Manual. Tallahassee: Psychology Department, Florida A \& M University, Tallahassee.

Baldwin, J. A., \& Bell, Y. R. (1985). The African Self-Consciousness Scale: An Africentric Personality Questionnaire. Western Journal of Black Studies, 9, 6168 .

Barrett-Lennard, G. T. (1962). Dimensions of therapist response as causal factors in therapeutic change. Psychological Monographs, 76, 1-36.

Baugh, E. (2005). Relationship between ethnic identity and eating disorder behaviors and attitudes among African American and European American college females. Unpublished doctoral dissertation, Florida State University, Tallahassee. 
Befort, C. A., Thomas, J. L., Daley, C. M., Rhode, P. C., \& Ahluwalia, J. S. (2008). Perceptions and beliefs about body size, weight, and weight loss among obese African American women: A qualitative inquiry. Health Education \& Behavior, $35,410-426$.

Bentler, P. M. (1990). Comparative fit indexes in structural models. Psychological Bulletin, 107, 238-246.

Betz, N. E., \& Campbell, C. (2003). Self-efficacy and personality correlates of instrumentality. Unpublished manuscript.

Betz, N. E., Wohlgemuth, E., Serling, D., Harshbarger, J., \& Klein, K. (1995). Evaluation of a measure of self-esteem based on the concept of unconditional self-regard. Journal of Counseling \& Development, 74, 76-83.

Birnbaum, A. (1991). Measuring level of ethnic identity: A comparison of two new scales. Unpublished doctoral dissertation, New Brunswick, NJ, Rutgers University.

Buchanan, T. S., Fischer, A. R., Tokar, D. M., \& Yoder, J. D. (2008). Testing a culturespecific extension of objectification theory regarding African American women's body image. The Counseling Psychologist, 36, 697-718.

Byrne, B. N. (2001). Structural equation modeling with AMOS: Basic concepts and programming. Mahwah, NJ: Lawrence Erlbaum Associates.

Cachelin, F. M., \& Regan, P. C. (2006). Prevalence and correlates of chronic dieting in a multi-ethnic U.S. community sample. Eating and Weight Disorders, 11, 91-99.

Cachelin, F. M., Veisel, C., Barzegarnazari, E., \& Striegel-Moore, R. H. (2000). Disordered eating, acculturation, and treatment seeking in a community sample of Hispanic, Asian, Black, and White women. Psychology of Women Quarterly, 24, 244-253.

Caldwell, M. B., Brownell, K. D., \& Wilfley, D. E. (1997). Relationship of weight, body dissatisfaction, and self-esteem in African American and White female dieters. International Journal of Eating Disorders, 22, 127-130.

Canadian Dietetic Association. (1988). The body test material. Toronto: Canadian Dietetic Association. 
Carper, J. L., Fisher, J. O., \& Birch, L. L. (2000). Young girls' emerging dietary restraint and disinhibition are related to parental control in child feeding. Appetite, 35, 121-129.

Carrier, K. M., Steinhardt, M. A., \& Bowman, S. (1994). Rethinking traditional weight management programs: A 3-year follow-up evaluation of a new approach. The Journal of Psychology, 128, 517-535.

Cash, T. F. (1997). Cognitive-behavioral perspectives on body image. In T. F. Cash, \& T. Pruzinsky (Eds.), Body image: A handbook of theory, research, and clinical practice (pp. 38-46). New York: Guilford Press.

Cash, T. F., Winstead, B. A., \& Janda, L. H. (1986). The great American shape-up. Psychology Today, 20, 30-37.

Cashel, M. L., Cunningham, D., Landeros, C., Cokley, K. O., \& Muhammad, G. (2003). Sociocultural attitudes and symptoms of bulimia: Evaluating the SATAQ with diverse college groups. Journal of Counseling Psychology, 50, 287-296.

Choate, L. H. (2005). Toward a theoretical model of women' body image resilience. Journal of Counseling \& Development, 83, 320-330.

Claiborn, C. D., Crawford, J. B., \& Hackman, H. W. (1983). Effects of intervention discrepancy in counseling for negative emotions. Journal of Counseling Psychology, 30, 164-171.

Cokley, K. O. (2002). Testing Cross's revised racial identity model: An examination of the relationship between racial identity and internalized racialism. Journal of Counseling Psychology, 49, 476-483.

Cokley, K. O. (2005). Racial(ized) identity, ethnic identity, and Afrocentric values: Conceptual and methodological challenges in understanding African American Identity. Journal of Counseling Psychology, 52, 517-526.

Cokley, K. O. (2007). Critical issues in the measurement of ethnic and racial identity: A referendum on the state of the field. Journal of Counseling Psychology, 54, 224234.

Cole, R. E. (2006). Intuitive Eating Non-Dieting Approach to Weight Management: A pilot Study with Fort Drum Health Beneficiaries. UMI\#3251799. UMI Dissertation Services, ProQuest Co.: Ann Arbor, MI. 
Constantine, M. G., \& Sue, D. W. (2006). Factors contributing to optimal human functioning in people of color in the United States. The Counseling Psychologist, $34,228-244$.

Cooper, P. J., Taylor, M. J., Cooper, Z., \& Fairburn, C. G. (1987). The development and validation of the Body Shape Questionnaire. International Journal of Eating Disorders, 6, 485-494.

Cross, W. E., Jr. (1978). The Thomas and Cross models of psychological nigrescence: A review. Journal of Black Psychology, 5, 13-31.

Cross, W. E., Jr. (1991). Shades of black: Diversity in African-American identity. Philadelphia: Temple University Press.

Cross, W. E., Jr. (1971). The negro to black conversion experience: Toward a psychology of Black liberation. Black World, 20, 13-27.

Cross, W. E., Jr., \& Vandiver, B. J. (2001). Nigrescence theory and measurement: Introducing the Cross Racial Identity Scale (CRIS). In J. G. Ponterotto, J. M. Casas, L. A. Suzuki, \& C. M. Alexander (Eds.), Handbook of multicultural counseling (2nd ed., pp. 371-393). Thousand Oaks, CA: Sage.

Cuellar, I., Arnold, B., \& Maldonado, R. (1995). Acculturation rating scale for Mexican Americans-II: A revision of the original ARSMA scale. Hispanic Journal of Behavioral Science, 17, 275-304.

Cutrona, C. E., \& Russell, D. (1987). The provisions of social relationships and adaptation to stress. In W. H. Jones \& D. Perlman (Eds.), Advances in personal relationships (pp. 37-67). Greenwich, CT: JAI Press.

Davis, N. L., Clance, P. R., \& Gailis, A. T. (1999). Treatment approaches for obese and overweight African American women: A consideration of cultural dimensions. Psychotherapy, 36, 27-35.

Diener, E., Emmons, R. A., Larsen, R. J., \& Griffin, S. (1985). The Satisfaction with Life Scale. Journal of Personality Assessment, 49, 71-75.

Dinsmore, B. D., \& Mallinckrodt, B. (1996). Emotional self-awareness, eating disorders, and racial identity attitudes in African American women. Journal of Multicultural Counseling and Development, 24, 267-277. 
Dutton, G. R., Martin, P. D., \& Brantley, P. J. (2004). Ideal weight goals of African American women participating in a weight management program. Body Image, 1, 305-310.

D’Zurilla, T. J., Nezu, A. M., \& Maydeu-Olivares, A. (1997). Manual for the Social Problem-Solving Inventory - Revised. North Tonawanda, NY: Multi-Health Systems.

Edwards-Hewitt, T., \& Gray, J. J. (1993). The prevalence of disordered eating attitudes and behaviours in Black-American and White-America college women: Ethnic, regional, class, and media differences. Eating Disorder Review, 1, 41-52.

Fairburn, C. G., \& Wilson, G. T. (1993). Binge eating: Nature assessment, and treatment. New York: Guilford Press.

Faith, M. S., Scanlon, K. S., Birch, L. L., Francis, L. A., \& Sherry, B. (2004). Parentchild feeding strategies and their relationship to child eating and weight status. Obesity Research, 12, 1711-1722.

Falconer, J. W., \& Neville, H. (2000). African American college women's body image: An examination of body mass, African self-consciousness, and skin color satisfaction. Psychology of Women Quarterly, 24, 236-243.

Fischer, A. R., Tokar, D. M., \& Serna, G. S. (1998). Validity and construct contamination of the Racial Identity Attitudes Scale-Long Form. Journal of Counseling Psychology, 45, 212-224.

Franko, D. L., Becker, A. E., Thomas, J. J., \& Herzog, D. B. (2007). Cross-ethnic differences in eating disorder symptoms and related distress. International Journal of Eating Disorders, 40, 156-164.

Franko, D. L., \& Edwards George, J. B. (2006). Eating disorders, culture, and ethnicity: Connections and challenges in group therapy. Group, 30, 307-320.

Franzoi, S. L., \& Shields, S. A. (1984). The Body Esteem Scale: Multidimensional structure and sex differences in a college population. Journal of Personality Assessment, 42, 173-178.

Fredrickson, B., \& Roberts, T. (1997). Objectification theory: Toward understanding women's lived experiences and mental health risks. Psychology of Women Quarterly, 21, 173-206. 
Freedman, E. K., Carter, M. M., Sbrocco, T., \& Gray, J. J. (2004). Ethnic differences in preferences for female weight and waist-to-hip ratio: A comparison of AfricanAmerican and White American college and community samples. Eating Behaviors, 5, 191-198.

Gardner-Kitt, D. L., \& Worrell, F. C. (2007). Measuring nigrescence attitudes in schoolaged adolescents. Journal of Adolescence, 30, 187-202.

Garner, D. M. (1991). Eating Disorder Inventory-2: Professional Manual. Odessa, Fl: Psychological Assessment Resources.

Garner, D. M. (2004). Eating Disorder Inventory-3 manual. Odessa, Fl: Psychological Assessment Resources.

Garner, D. M., \& Garfinkel, P. E. (1979). The Eating Attitudes Test: An index of the symptoms of anorexia nervosa. Psychological Medicine, 9, 273-279.

Garner, D. M., Olmsted, M. P., Bohr, Y., \& Garfinkel, P. E. (1982). The Eating Attitudes Test: Psychometric features and clinical correlates. Psychological Medicine, 12, 871-878.

Garner, D. M., Olmstead, M. P., \& Polivy, J. (1983). Development and validation of a multidimensional eating disorder inventory for anorexia nervosa and bulimia. International Journal of Eating Disorders, 2, 15-34.

Garner, D. M., Olmstead, M. P., \& Polivy, J. (1983). EDI. Odessa,FL: Psychological Assessment Resources, Inc.

Garner, D. M., \& Olmsted, M. P. (1984). Eating Disorder Inventory Manual. Odessa, Fl: Psychological Assessment Resources, Inc.

Gast, J. A., \& Hawks, S. R. (1998). Weight loss education: The challenge of a new paradigm. Health Education and Behavior, 25, 464-473.

Gast, J. A., \& Hawks, S. R. (2000). Examining intuitive eating as a weight loss program. Health Weight Journal, 14, 42-44.

Gelso, C. J., \& Fretz, B. R. (2001). Counseling psychology (2nd ed.). Fort Worth, TX: Harcourt. 
Golden, B. R., Buzcek, T., \& Robbins, S. A. (1986). Parameters of bulimia: Examining the compulsive eating scale. Measurement and Evaluation in Counseling and Development, 19, 84-92.

Goldfarb, L. A., Dykens, E. M., \& Gerrard, M. (1985). The Goldfarb Fear of Fat Scale. Journal of Personality Assessment, 49, 329-332.

Goodstein, R., \& Ponterotto, J. G. (1997). Racial and ethnic identity: Their relationship and their contribution to self-esteem. Journal of Black Psychology, 23, 275-292.

Gordon, K. H., Brattole, M. M., Wingate, L. R., \& Joiner, T. E. (2006). The impact of client race on clinician detection of eating disorders. Behavior Therapy, 37, 319325.

Gray, J. J., Ford, K., \& Kelly, L. M. (1987). The prevalence of bulimia in a Black college population. International Journal of Eating Disorders, 6, 733-740.

Greenberg, D. R., \& LaPorte, D. J. (1996). Racial differences in body type preferences of men for women. International Journal of Eating Disorders, 19, 275-278.

Greene, B. (1994). African-American women. In L. Comas-Diaz, \& B. Greene (Eds.), Women of color: Integrating ethnic and gender identities in psychotherapy (pp. 10-29). New York: Guilford.

Greenglass, E. R., Schwarzer, R., \& Taubert, S. (1999). The Proactive Coping Inventory (PCI): A multidimensional research instrument. Available at http://www.psych.yorkn.ca/greenglass/.

Gurman, A. S. (1977). The patient's perspective of the therapeutic relationship. In A. S. Gurman, \& A. M. Razin (Eds.), Effective psychotherapy: A handbook of research (pp. 503-543). New York: Pergamon Press.

Hall, C. C. (1995). Beauty is in the soul of the beholder: Psychological implications of beauty and African American women. Cultural Diversity \& Mental Health, 1, $125-137$.

Hargreaves, M. K., Schlundt, D. G., \& Maciej, S. (2002). Contextual factors influencing the eating behaviors of African American women: A focus group investigation. Ethnicity \& Health, 7, 133-147. 
Harris, A. H., Thoresen, C. E., \& Lopez, S. J. (2007). Integrating positive psychology into counseling: Why and (when appropriate) how. Journal of Counseling \& Development, 85, 3-13.

Harris, S. M. (1994). Racial differences in predictors of college women's body image attitudes. Women \& Health, 21, 89-104.

Harris, S. M. (1995). Family, self, and sociocultural contributions to body-image attitudes of African-American women. Psychology of Women Quarterly, 19, 129-145.

Hawkins, R. C., \& Clement, P. F. (1980). Development and construct validation of a selfreport measure of binge eating tendencies. Addictive Behaviors, 5, 219-226.

Hawks, S. R., Madanat, H. N., \& Harris, A. M. (2006). The relationship between intuitive eating and health indicators among college women. American Journal of Health Education, 26, 322-324.

Hawks, S. R., Madanat, H. N., Hawks, J., \& Harris, A. M. (2005). American Journal of Health Education, 36, 331-336.

Hawks, S. R., Merrill, R. M., \& Madanat, H. N. (2004). The Intuitive Eating Scale (IES). American Journal of Health Education, 35, 90-99.

Hawks, S. R., Merrill, R. M., Madanat, H. N., Miyagawa, T., Suwanteerangkul, J., Guarin, C. M., et al. (2004). Intuitive eating and the nutrition transition in Asia. Asian Pacific Journal of Clinical Nutrition, 13, 194-203.

Heinberg, L. J., Thompson, J. K., \& Stormer, S. (1995). Development and validation of the Sociocultural Attitudes Towards Appearance Questionnaire. International Journal of Eating Disorders, 17, 81-89.

Helm, K. M. (2002). A theoretical and psychometric analysis of the revised Black racial identity development model and the multidimensional model of racial identity: Outcomes on the Revised African American Acculturation Scale-33. Dissertation Abstracts International: Section B: The Sciences and Engineering, 62 (10-B), 4833.

Helms, J. E. (1990). Black and White racial identity. Westport, CT: Greenwood Press. 
Herman, C. P., et al. (1978). Distractibility in dieters and nondieters: An alternative view of "externality". Journal of Personality and Social Psychology, 36, 536-548.

Herman, C. P., \& Mack, D. (1975). Restrained and unrestrained eating. Journal of Personality, 43, 647-660.

Herman, C. P., \& Polivy, J. (2005). Normative influences on food intake. Physiology and Behavior, 86, 762-772.

Herman, C. P., Polivy, J., Lank, C. N., \& Heatherton, T. F. (1987). Anxiety, hunger, and eating behavior. Journal of Abnormal Psychology, 96, 264-269.

Hollingshead, A. B. (1957). Two-factor index of social position. New Haven, CT: Yale Station.

Hopkins, S., Burrows, E., Bowen, D. J., \& Tinker, L. F. (2001). Differences in eating pattern labels between maintainers and nonmaintainers in the women's health initiative. Journal of Nutrition Education, 33, 278-283.

Hu, L., \& Bentler, P. (1999). Cutoff criteria for fit indices in covariance structure analysis: Conventional criteria versus new alternatives. Structural Equation Modeling, 6, 1-55.

James, D. C. (2004). Factors influencing food choices, dietary intake, and nutritionrelated attitudes among African Americans: Applications of a culturally sensitive model. Ethnicity \& Health, 9, 349-367.

Johnson, C. (1984). Initial consultation for patients with bulimia and anorexia nervosa. In D. M. Garner, \& P. E. Garfinkel (Eds.), Anorexia nervosa and bulimia (pp. 1955). New York: The Guilford Press.

Jones, H. L., Cross, W. E., Jr., \& DeFour, D. C. (2007). Race-related stress, racial identity attitudes, and mental health among Black women. Journal of Black Psychology, 33, 208-231.

Kaplan, R. M. (2007). Should Medicare reimburse providers for weight loss interventions? American Psychologist, 62, 217-219.

Klonoff, E. A., \& Landrine, H. (1995). The Schedule of Sexist Events: A measure of lifetime and recent sexist discrimination in women's lives. Psychology of Women Quarterly, 19, 439-472. 
Klonoff, E. A., \& Landrine, H. (1999). Cross-validation of the Schedule of Racist Events. Journal of Black Psychology, 25, 231-254.

Kumanyika, S. K., Morssink, C., \& Agurs, T. (1992). Models for dietary and weight change in African-American women: Identifying cultural components. Ethnicity and Disease, 2, 166-175.

Landrine, H., \& Klonoff, E. A. (1994). The African American Acculturation Scale: Development, reliability, and validity. Journal of Black Psychology, 20, 104-127.

Landrine, H., \& Klonoff, E. A. (1996). The Schedule of Racist Events: A measure of racial discrimination and a study of its negative physical and mental health consequences. Journal of Black Psychology, 22, 144-168.

Lemon, R. L., \& Waehler, C. A. (1996). A test of the stability and construct validity of the Black Racial Identity Scale, Form B (RIAS-B) and the White Racial Identity Scale (WRIAS). Measurement and Evaluation in Counseling and Development, $29,77-85$.

Lester, R., \& Petrie, T. A. (1998). Physical, psychological, and societal correlates of bulimic symptomatology among African American college women. Journal of Counseling Psychology, 35, 315-321.

Leibman,M. (2005). Promoting healthy weight: Lessons learned from WIN the Rockies and other key studies. Journal of Nutrition Education and Behavior, 37, 95-100.

Lopez, S. J., Magyar-Moe, J. L., Peterson, S. E., Ryder, J. A., Krieshok, T. S., O’Bryne, K. K., et al. (2006). Counseling psychology's focus on positive aspects of human functioning. The Counseling Psychologist, 34, 205-227.

Lovejoy, M. (2001). Disturbances in the social body: Differences in body image and eating problems among African American and White women. Gender \& Society, 15, 239-261.

Makkar, J. K., \& Strube, M. J. (1995). Black women's self-perceptions of attractiveness following exposure to White versus Black beauty standards: The moderating role of racial identity and self-esteem. Journal of Applied Social Psychology, 25, $1547-1566$. 
Mann, B., \& Murphy, K. C. (1975). Timing of self-disclosure, reciprocity of selfdisclosure, and reactions to an initial interview. Journal of Counseling Psychology, 22, 304-308.

Mann, T., Tomiyama, A., Westling, E., Lew, A., Samuels, B., \& Chatman, J. (2007). Medicare's search for effective obesity treatments: Diets are not the answer. American Psychologist, 62, 220-223.

Maris, R. Intuitive eating: Relearning how to eat for life. Center for Change [Website]. Available at: http://centerforchange.com/articles/detail.php?i=37. Accesses January 15, 2009.

McKinley, N. M., \& Hyde, J. S. (1996). The Objectified Body Consciousness Scale: Development and validation. Psychology of Women Quarterly, 20, 181-215.

Mendelson, B. K., Mendelson, M. J., White, D. R. (2001). Body-Esteem Scale for adolescents and adults. Journal of Personality Assessment, 76, 90-106.

Mills, D. H., \& Zytowski, D. G. (1967). Healing relationship: A structural analysis. Journal of Counseling Psychology, 14, 193-197.

Molloy, B. L., \& Herzberger, S. D. (1998). Body image and self-esteem: A comparison of African American and Caucasian women. Sex Roles, 38, 631-642.

Moradi, B., Dirks, D., \& Matteson, A. V. (2005). Roles of sexual objectification experiences and internalization of standards of beauty in eating disorder symptomatology: A test and extension of objectification theory. Journal of Counseling Psychology, 52, 420-425.

Moradi, B., \& Rottenstein, A. (2007). Objectification theory and deaf cultural identity attitudes: Roles in deaf women's eating disorder symptomatology. Journal of Counseling Psychology, 54, 178-188.

Mulholland, A. M., \& Mintz, L. B. (2001). Prevalence of eating disorders among African American women. Journal of Counseling Psychology, 48, 111-116.

Muthen, L. K., \& Muthen, B. O. (2006). Mplus user's guide (4th ed.). Los Angeles: Muthen \& Muthen.

O’Neill, S. K. (2003). African American women and eating disturbance: A meta-analysis. Journal of Black Psychology, 29, 3-16. 
Osvold, L. L., \& Sodowsky, G. R. (1993). Eating disorders of White American, racial and ethnic minority American, and international women. Journal of Multicultural Counseling and Development, 21, 143-154.

Parham, T., \& Helms, J. (1981). The influence of Black student's racial identity attitudes on preference for counselor's race. Journal of Counseling Psychology, 28, 250257.

Parker, S., Nichter, M., Nichter, N., Vuckovic, N., Sims, C., \& Ritenbaugh, C. (1995). Body image and weight concerns among African American and White adolescent females: Differences that make a difference. Human Organization, 54, 103-114.

Paulus,D. L. (1994). Balanced Inventory of Desirable Responding reference manual for BIDR Version 6. Vancouver, British Columbia, Canada: University of British Columbia.

Pedhazur, E. J., \& Schmelkin, L. P. (1991). Measurement, design, and analysis: An integrated approach. Hillsdale, NJ: Lawrence Erlbaum.

Peterson, M., Rojhani, A., Steinhaus, N., \& Larkin, B. (2000). Effects of ethnic identity on attitude, feelings, and behaviors toward food. Eating Disorders, 8, 207-219.

Phinney, J. S. (1992). The Multigroup Ethnic Identity Measure: A new scale for use with adolescent and young adults from diverse groups. Journal of Adolescent Research, 7, 156-176.

Phinney, J. S. (1996). Understanding ethnic diversity: The role of ethnic identity. American Behavioral Scientist, 40, 143-152.

Phinney, J. S., \& Alipuria, L. (1990). Ethnic identity in college students from four ethnic groups. Journal of Adolescent Research, 13, 171-183.

Phinney, J. S., Chavira, V., \& Tate, J. D. (1996). The effect of ethnic threat on ethnic self-concept and own-group ratings. Journal of Social Psychology, 133, 469-478.

Phinney, J. S., \& Onwugalu,M. (1996). Racial identity and perceptions of American ideals among African Americans and African students in the United States. International Journal of Intercultural Relations, 20, 127-140. 
Plemmer, J. (2005). Racial identity and sociocultural attitudes as predictors of eating disturbance among African-American women. Unpublished doctoral dissertation, Kent State University.

Polivy, J., Coleman, J., \& Herman, C. P. (2005). The effect of deprivation on food cravings and eating behavior in restrained and unrestrained eaters. International Journal of Eating Disorders, 38, 301-309.

Polivy, J., \& Herman, C. P. (1987). Diagnosis and treatment of normal eating. Journal of Consulting and Clinical Psychology, 55, 635-644.

Polivy, J., \& Herman, C. P. (1992). Undieting: A program to help people stop dieting. International Journal of Eating Disorders, 11, 261-268.

Polivy, J., \& Herman, C. P. (1999). Distress and eating: Why do dieters overeat? International Journal of Eating Disorders, 26, 153-164.

Ponterotto, J. G., Baluch, S., Greig, T., Rivera, L. (1998). Development and initial score validation of the Teacher Multicultural Attitude Survey. Educational and Psychological Measurement, 58, 1002-1016.

Ponterotto, J. G., Gretchen, D., Utsey, S. O., Stracuzzi, T., \& Saya, R. (2003). The Multigroup Ethnic Identity Measure (MEIM): Psychometric review and further validity testing. Educational and Psychological Measurement, 63, 502-515.

Powell, A. D., \& Kahn, A. S. (1995). Racial differences in women's desires to be thin. International Journal of Eating Disorders, 17, 191-195.

Powell, L., Calvin, J., \& Calvin, J. E. (2007). Effective obesity treatments. American Psychologist, 62, 234-246.

Preachers, K. J., \& Hayes, A. F. (2004). SPSS and SAS procedures for estimating indirect effects in simple mediations models. Behaviors Research Methods, Instruments, and Computers, 36, 717-731.

Pumariega, A. J. (1986). Acculturation and eating attitudes in adolescent girls: A comparative and correlational study. Journal of the American Academy of Child and Adolescent Psychiatry, 25, 276-279.

Quintana, S. M. (2007). Racial and ethnic identity: Developmental perspectives and research. Journal of Counseling Psychology, 54, 259-270. 
Regan, P. C., \& Cachelin, F. M. (2006). Binge eating and purging in a multi-ethnic community sample. International Journal of Eating Disorders, 39, 523-526.

Rogers, J. R., \& Abbey-Hines, J. (1997). Confirmatory factor analysis of the gender role conflict scale: A cross-validation of Good et al., 1995. Measurement \& Evaluation in Counseling and Development, 30, 137-146.

Rogers, C. R. (1961). On becoming a person. Boston, MA: Houghton Mifflin.

Rogers, C. R. (1964). Toward a modem approach to values: The valuing process in the mature person. Journal of Abnormal and Social Psychology, 68, 160-167.

Rosenberg, M. (1965). Society and the adolescent self-image. Princeton, NJ: Princeton University Press.

Rucker, C. E., \& Cash, T. F. (1992). Body images, body-size perceptions, and eating behaviors among African-American and White college women. International Journal of Eating Disorders, 12, 291-299.

Russell, D., \& Cutrona, C. (1984). The provision of social relationships and adaptation to stress. Paper presented at the American Psychological Association, Toronto, ON, Canada.

Sabik, N., Cole, E. R., \& Ward., L. M. (2007). Assessing body satisfaction among Black, Asian Americans, and White women. Paper presented at the annual convention of the American Psychological Association, San Francisco, CA.

Savoye, M., Berry, D., Dziura, J., Shaw., M., Serrechia., J., \& Fruhart, J. (2002). Antropometric and psychosocial changes in obese adolescents enrolled in a weight management program. Journal of the American Dietetic Association, 105, 364-370.

Scheier, M. F., Carver, C. S., \& Bridges, M. W. (1994). Distinguishing optimism from neuroticism (and trait anxiety, self-mastery, and self-esteem): A reevaluation of the Life Orientation Test. Journal of Personality and Social Psychology, 67, 1063-1078.

Seligman, M. E., \& Csikszentmihalyi, M. (2000). Positive psychology: An introduction. American Psychologist, 55, 5-14. 
Shrout, P. E., \& Bolger, N. (2002). Mediation in experimental and non-experimental studies: New procedures and recommendations. Psychological Methods, 7, 422445.

Simmons, J., Roberge, L., Kendrick, S. B., \& Richards, B. (1995). The interpersonal relationship in clinical practice: The Barrett-Lennard Relationship Inventory as an assessment instrument. Evaluation and the Health Professions, 18, 103-112.

Smith, E. J. (2006). The strength-based counseling model. The Counseling Psychologist, $34,13-79$.

Smith, T., \& Hawks, S. R. (2006). Intuitive eating, diet composition, and the meaning of food in healthy weight promotion. American Journal of Health Education, 37, 130-136.

Spadafore, J. (2008). Disordered eating in African American women: An object relations perspective. Unpublished doctoral dissertation. Tennessee State University.

Steinhardt, M. A., Bezner, J. R., \& Adams, T. B. (1999). Outcomes of a traditional weight control program and a nondiet alternative: A one-year comparison. The Journal of Psychology, 133, 495-513.

Stice, E., Nemeroff, C., \& Shaw, H. (1996). Test of the dual pathway model of bulimia nervosa: Evidence for dietary restraint and affect regulation mechanisms. Journal of Social and Clinical Psychology, 15, 340-363.

Stice, E., Ziemba, C., Margolis, J., \& Flick, P. (1996). The dual pathway model differentiates bulimics, subclinical bulimics and controls: Testing the continuity hypothesis. Behavior Therapy, 27, 531-549.

Striegel-Moore, R. H., \& Bulik, C. M. (2007). Risk factors for eating disorders. American Psychologist, 62, 181-198.

Striegel-Moore, R. H., \& Smolak, L. (1996). The role of race in the development of eating disorders. In L. Smolak, M. P. Levine \& R. Striegel-Moore (Eds.), The developmental psychopathology of eating disorders (pp. 259-284). Mahwah, NJ: Lawrence Erlbaum Associates.

Striegel-Moore, R. H., \& Smolak, L. (2000). The influence of ethnicity on eating disorders in women. In R. M. Eisler, \& M. Hersen (Eds.), Handbook of gender, culture, and health (pp. 227-253). Mahwah, NJ: Lawrence Erlbaum Associates. 
Suinn, R. M., Ahuna, C., \& Khoo, G. (1992). The Suinn-Lew Asian Self-Identity Acculturation Scale: Concurrent and factorial validation. Educational and Psychological Measurement, 52, 1041-1046.

Swami, V., \& Chamorro-Premuzic, T. (2008). Factor structure of the Body Appreciation Scale among Malaysian women. Body Image, 5, 409-413.

Swami, V., Stieger, S., Haubner, T., \& Voracek, M. (2008). Translation and validation of the German Body Appreciation Scale. Body Image, 5, 122-127.

Swami, V., Hadji-Michael, M., Furnham, A.(2008). Personality and individual difference correlates of positive body image. Body Image, 5, 322-325.

Tabachnick, B. G., \& Fidell, L. S. (1996). Using multivariate statistics (3rd ed.). New York: HarperCollins.

Tabachnick, B. G., \& Fidell, L. S. (2001). Using multivariate statistics (4th ed.). New York: HarperCollins.

Talleyrand, R. M. (2002). Racial identity schemas as mediators of the relationship between perceived racial stressors and disordered eating in Black women. Dissertation Abstracts International: Section B: The Sciences \& Engineering, 62, 4239.

Talleyrand, R. M. P. (2006). Potential stressors contributing to eating disorder symptoms in African American Women: Implications for mental health counselors. Journal of Mental Health Counseling, 28, 338-352.

Taub, D. J. (1995). Relationship of selected factors to traditional-age undergraduate women's development of autonomy. Journal of College Student Development, 36, $141-151$.

Taylor, J. Y., Caldwell, C. H., Baser, R. E., Faison, N., \& Jackson, J. S. (2007). Prevalence of eating disorders among Blacks in the national survey of American life. International Journal of Eating Disorders, 40, 10-14.

Thomas, V. G. (2001). Body-image satisfaction among Black women. The Journal of Social Psychology, 129, 107-112. 
Thompson, J. K., Fabian, L. J., Moulton, D. O., Dunn, M. E., \& Altabe, M. N. (1991). Development and validation of the Physical Appearance Related Teasing Scale (PARTS). Journal of Personality Assessment, 56, 513-521.

Todman, J., \& Dugard, P. (2007). Approaching multivariate analysis: An introduction for psychology. New York: Psychology Press.

Tokar, D. M., \& Ficher, A. R. (1998). Psychometric analysis of the Racial Identity Attitudes Scale-Long Form. Measurement and Evaluation in Counseling and Development, 31, 138-149.

Tribole, E., \& Resch, E. (1995). Intuitive eating a recovery book for the chronic dieter. New York: St. Martin's Press.

Tylka, T. L. (2006). Development and psychometric evaluation of a measure of intuitive eating. Journal of Counseling Psychology, 53, 226-240.

Tylka, T. L. (2007). Roles of self-esteem and proactive coping in fostering intuitive eating. Paper presented at the annual convention of the American Psychological Association, San Francisco, CA.

Tylka, T. L., \& Hill, M. S. (2004). Objectification theory as it relates to disordered eating among college women. Sex Roles, 51, 719-730.

Tylka, T. L., \& Subich, L. M. (1999). Exploring the construct validity of the eating disorder continuum. Journal of Counseling Psychology, 46, 268-276.

Tylka, T. L., \& Subich, L. M. (2003). Revisiting the latent structure of eating disorders: Taxometric analyses with nonbehavioral indicators. Journal of Counseling Psychology, 50, 276-286.

Tylka, T. L., \& Subich, L. M. (2004). Examining a multidimensional model of eating disorder symptomatology among college women. Journal of Counseling Psychology, 51, 314-328.

Tylka, T. L., \& Wilcox, J. A. (2006). Are intuitive eating and eating disorder symptomatology opposite poles of the same construct? Journal of Counseling Psychology, 53, 474-485. 
Vander Wal, J. S., \& Thomas, N. (2004). Predictors of body image dissatisfaction and disturbed eating attitudes and behaviors in African American and Hispanic girls. Eating Behaviors, 5, 291-301.

Vandiver, B. J., Cross, W. E., Jr., Fhagen-Smith, P. E., Worrell, F. C., Swim, J. K., \& Caldwell, L. D. (2000). The Cross Racial Identity Scale, State College, PA: Author.

Vandiver, B. J., Cross, W. E., Jr., Worrell, F. C., Fhagen-Smith, P. E. (2002). Validating the Cross Racial Identity Scale. Journal of Counseling Psychology, 49, 71-85.

Vaughan, C. A., Sacco, W. P., \& Beckstead, J. W. (2008). Racial/ethnic differences in body mass index: The roles of beliefs about thinness and dietary restriction. Body Image, 5, 291-301.

Vaughan, C. A., Stewart, V., \& Sacco, W. P. (2004). Ethnicity moderates the relationship between body mass index and relationship status. Poster session presented at the annual convention of the American Psychological Association, Honolulu, HI.

Wade, T. J., \& DiMaria, C. (2003). Weight halo effects: Individual difference in perceived life success as a function of women's race and weight. Sex Roles: A Journal of Research, 48, 461-465.

Wade, T. J., Loyden, J., Renninger, L., \& Tobey, L. (2003). Weight halo effects: Individual differences in personality evaluations as a function of weight. Personality and Individual Differences, 34, 263-268.

Watkins, C. E. (1983). Counseling psychology versus clinical psychology: Further explorations on a theme or once more around the "identity" maypole with gusto. The Counseling Psychologist, 11, 76-92.

Watson, D., Clark, L. A., \& Tellegen, A. (1988). Development and evaluation of a brief measure of positive and negative affect: The PANAS scales. Journal of Personality and Social Psychology, 54, 1063-1070.

Wilcox, J. A. (2006). Construct validity for the Barrett-Lennard Relationship Inventory with college women. Unpublished manuscript. 
Wilcox, J. A. (2007). Toward an understanding of resilience to disordered eating and body image dissatisfaction among African American women: An analysis of the roles of ethnicity and feminist identities. Unpublished doctoral dissertation. The Ohio State University.

Wildes, J. E., Emery, R. E., \& Simons, A. D. (2001). The roles of ethnicity and culture in the development of eating disturbance and body dissatisfaction: A meta-analytic review. Clinical Psychology Review, 21, 521-551.

Wilfley, D. E., Schreiber, G. B., Pike, K. M., Striegel-Moore, R. H., Wright, D. J., \& Rodin, J. (1996). Eating disturbance and body image: A comparison of a community sample of adult Black and White women. International Journal of Eating Disorders, 20, 377-387.

Wonderlich, S. A., Joiner, T. E., Keel, P. K., Williamson, D. A., \& Crosby, R. D. (2007). Eating disorder diagnoses: Empirical approaches to classification. American Psychologist, 62, 167-180.

Wood-Barcalow, N. (2006). Understanding the construct of body image to include positive components: A mixed-method study. Unpublished doctoral dissertation, The Ohio State University.

Worrell, F. C. (2000). A validity study of scores on the Multigroup Ethnic Identity Measure based on a sample of academically talented adolescents. Education and Psychological Measurements, 60, 439-447.

Worrell, F. C., \& Gardner-Kitt, D. L. (2006). The relationship between racial and ethnic identity in black adolescents: The Cross Racial Identity Scale (CRIS) and the Multigroup Ethnic Identity Measure (MEIM). Identity: An International Journal of Theory and Research, 6, 293-315.

Worrell, F. C., Vandiver, B. J., \& Cross, W. E. (2004). The Cross Racial Identity Scale: Technical manual (2nd ed.). Berkeley, CA: Author.

Worrell, F. C., \& Watson, S. (2008). A confirmatory factor analysis of Cross Racial Identity Scale (CRIS) scores: Testing the expanded nigrescence model. Educational and Psychological Measurements, 68, 1041-1058. 
APPENDICES 


\section{APPENDIX A \\ DEMOGRAPHIC INFORMATION}

Directions for participants: For each item, please enter the information that describes you.

1. Sex:
a. Male
b. Female
c. Other (please describe)

2. Age:

3. Ethnic Identification:
a. African American
b. Biracial
c. Other (please describe)

4. Sexual Orientation
a. Exclusively homosexual
b. Primarily homosexual
c. Bisexual
d. Primarily heterosexual
e. Exclusively heterosexual
f. Other (please describe)
g. Uncertain

5. Relationship Status:
a. Not Dating
b. Casually Dating
c. Exclusive Dating Relationship
d. Marriage/ Partnership
e. Separated
f. Divorced/Widowed
g. Other (Please describe) 
6. Year in School
a. High School Student
b. Freshman
c. Sophomore
d. Junior
e. Senior
f. Post-Bac
g. Graduate Student
h. Other (please describe)

7. Socio-economic identification
a. Lower Class
b. Working Class
c. Middle Class
d. Upper-Middle Class
e. Upper Class
f. Other (please describe) 


\section{APPENDIX B}

\section{BARRETT-LENNARD RELATIONSHIP INVENTORY REVISED}

Directions for participants: In this section, please think about the most important/influential persons in your life as you were growing up. From the following list, please select those you consider to be important persons in your life as you were growing up (e.g., Mother, Father, Grandmother, Grandfather, Brother, Sister, Uncle, Aunt, Cousin, Teacher, Coach, Friend, Employer/Supervisor, Counselor, Clergy). Please note that if there are people that were important to you that are not listed, please write these persons in the space provided. In the space provided, please fill in all important/influential persons as you were growing up. Most Important Persons

Think about these people collectively and answer the following questions. Please consider each statement as being preceded by the statement, "While growing up..." For example, "While growing up, they respected me as a person." Please answer based on your level of agreement with each statement.

Please circle the corresponding number that indicates how strongly you feel the statement is true or false, in this relationship.

1) They respected me as a person.

$\begin{array}{cccccc}1 & 2 & 4 & 5 & { }^{6} \\ \text { Very False } & \text { Moderately False } & \text { Slightly False } & \text { Slightly True } & \text { Moderately True } & \text { Very True }\end{array}$

2) Their interest in me has depended on the things I have said or done.

$\begin{array}{cccccc}1 & 2 & 4 & 5 & 6 \\ \text { Very False } & \text { Moderately False } & \text { Slightly False } & \text { Slightly True } & \text { Moderately True } & \text { Very True }\end{array}$

3) They felt a true liking for me.

$\begin{array}{cccccc}1 & 2 & 3 & 5 & 6 \\ \text { Very False } & \text { Moderately False } & \text { Slightly False } & \text { Slightly True } & \text { Moderately True } & \text { Very True }\end{array}$

4) Whether I have felt happy or unhappy with myself has made no real difference to the way they have felt about me.

$\begin{array}{cccccc}1 & 2 & 4 & 5 & 6 \\ \text { Very False } & \text { Moderately False } & \text { Slightly False } & \text { Slightly True } & \text { Moderately True } & \text { Very True }\end{array}$ 
5) They been impatient with me.

$\begin{array}{cccccc}1 & & 2 & & & \\ \text { Very False } & \text { Moderately False } & \text { Slightly False } & \text { Slightly True } & \text { Moderately True } & \text { Very True }\end{array}$

6) Depending on my behavior, they had a better opinion of me sometimes than they had at other times.

$\begin{array}{cccccc}1 & 2 & 4 & 5 & { }^{6} \\ \text { Very False } & \text { Moderately False } & \text { Slightly False } & \text { Slightly True } & \text { Moderately True } & \text { Very True }\end{array}$

7) I have felt appreciated by them.

$\begin{array}{ccrccc}1 & & & & \\ \text { Very False } & \text { Moderately False } & \text { Slightly False } & \text { Slightly True } & \text { Moderately True } & \text { Very True }\end{array}$

8) Their feeling toward me hasn't depended on how I have felt toward them.

\section{$\begin{array}{llllll}\text { Very False } & \text { Moderately False } & \text { Slightly False } & \text { Slightly True } & \text { Moderately True } & \text { Very True }\end{array}$}

9) They have been indifferent to me.

$\begin{array}{cccccc}1 & 2 & 3 & 5 & 6 \\ \text { Very False } & \text { Moderately False } & \text { Slightly False } & \text { Slightly True } & \text { Moderately True } & \text { Very True }\end{array}$

10) They have wanted me to be a particular kind of person.

$\begin{array}{cccccc}1 & 2 & 4 & 5 & { }^{6} \\ \text { Very False } & \text { Moderately False } & \text { Slightly False } & \text { Slightly True } & \text { Moderately True } & \text { Very True }\end{array}$

11) They have found me rather dull and uninteresting.

$\begin{array}{cccccc}1 & & 2 & & & \\ \text { Very False } & \text { Moderately False } & \text { Slightly False } & \text { Slightly True } & \text { Moderately True } & \text { Very True }\end{array}$

12) I could have been openly critical or appreciative of them without really making them feel any different about me.

\begin{tabular}{|c|c|c|c|c|}
\hline 1 & 2 & 3 & 4 & 5 \\
\hline Fal & Moderately False & Slightly False & Slightly True & Moderately True \\
\hline
\end{tabular}

13) They have cared for me.

$\begin{array}{cccccc}1 & 2 & 3 & 5 & 6 \\ \text { Very False } & \text { Moderately False } & \text { Slightly False } & \text { Slightly True } & \text { Moderately True } & \text { Very True }\end{array}$

14) They have liked certain things about me, and there are other things they have not liked.

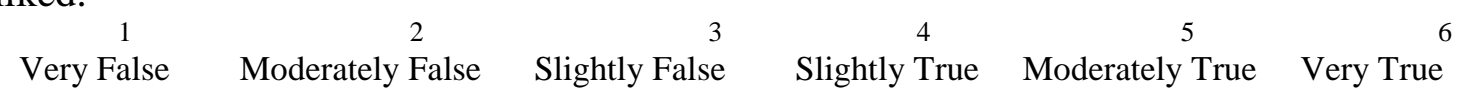

15) I have felt that they disapproved of me.
Very False
Moderately False Slightly False
$\begin{array}{ccr}4 & 5 & { }^{6} \\ \text { Slightly True } & \text { Moderately True } & \text { Very True }\end{array}$ 
16) Their attitude toward me has stayed the same; they have not been pleased with me sometimes and critical or disappointed at other times.

$\begin{array}{ccrrrr}1 & 2 & 4 & 5 & 6 \\ \text { Very False } & \text { Moderately False } & \text { Slightly False } & \text { Slightly True } & \text { Moderately True } & \text { Very True }\end{array}$

17) They have just tolerated me.

$\begin{array}{cccccc}1 & 2 & 4 & 5 & 6 \\ \text { Very False } & \text { Moderately False } & \text { Slightly False } & \text { Slightly True } & \text { Moderately True } & \text { Very True }\end{array}$

18) If I have shown that I have been angry with them, they have become hurt or angry with me too.

$\begin{array}{ccrccc}1 & 2 & & 4 & 5 & { }^{6} \\ \text { Very False } & \text { Moderately False } & \text { Slightly False } & \text { Slightly True } & \text { Moderately True } & \text { Very True }\end{array}$

19) They have been friendly and warm with me.

$\begin{array}{cccccc}1 & & 2 & & & \\ \text { Very False } & \text { Moderately False } & \text { Slightly False } & \text { Slightly True } & \text { Moderately True } & \text { Very True }\end{array}$

20) How much they have liked or disliked me has not been altered by anything that I have told them about myself.

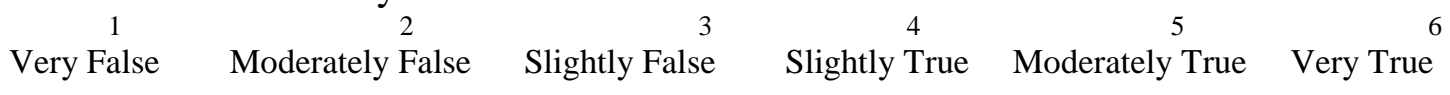

21) I have felt they have really valued me.

$\begin{array}{cccccc}1 & & 2 & & & \\ \text { Very False } & \text { Moderately False } & \text { Slightly False } & \text { Slightly True } & \text { Moderately True } & \text { Very True }\end{array}$

22) They have approved of some things I do, and plainly have disapproved of others.

$\begin{array}{cccccc}1 & 2 & 3 & 5 & 6 \\ \text { Very False } & \text { Moderately False } & \text { Slightly False } & \text { Slightly True } & \text { Moderately True } & \text { Very True }\end{array}$

23) They haven't liked me for myself.

$\begin{array}{cccccc}1 & 2 & & & & \\ \text { Very False } & \text { Moderately False } & \text { Slightly False } & \text { Slightly True } & \text { Moderately True } & \text { Very True }\end{array}$

24) Whether I have been in good spirits or feeling upset haven't made them feel any more or less appreciative of me.

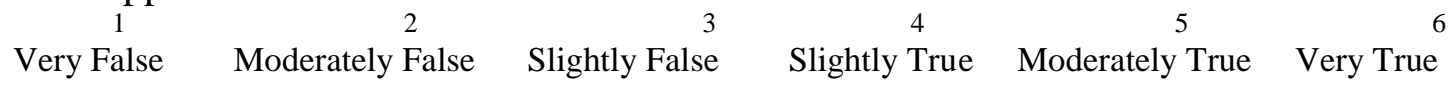

25) I seemed to have irritated and bothered them.

$\begin{array}{cccccc}1 & 2 & 4 & 5 & 6 \\ \text { Very False } & \text { Moderately False } & \text { Slightly False } & \text { Slightly True } & \text { Moderately True } & \text { Very True }\end{array}$


26) Whether the ideas and feelings I have expressed are good or bad have seemed to have made no difference to their feeling toward me.

$\begin{array}{cccccc}1 & & 2 & 4 & 5 & { }^{6} \\ \text { Very False } & \text { Moderately False } & \text { Slightly False } & \text { Slightly True } & \text { Moderately True } & \text { Very True }\end{array}$

27) At times they have felt contempt for me.

$\begin{array}{cccccc}1 & & 2 & 4 & 5 & 6 \\ \text { Very False } & \text { Moderately False } & \text { Slightly False } & \text { Slightly True } & \text { Moderately True } & \text { Very True }\end{array}$

28) Sometimes I have been more worthwhile in their eyes than I have been at other times.

$\begin{array}{cccccc}1 & 2 & 3 & 5 & 6 \\ \text { Very False } & \text { Moderately False } & \text { Slightly False } & \text { Slightly True } & \text { Moderately True } & \text { Very True }\end{array}$

29) They have been truly interested in me.

$\begin{array}{cccccc}1 & & 2 & 4 & 5 & 6 \\ \text { Very False } & \text { Moderately False } & \text { Slightly False } & \text { Slightly True } & \text { Moderately True } & \text { Very True }\end{array}$

30) I don't think that anything I have said or done has really changed the way they have felt toward me.

$\begin{array}{cccccc}1 & 2 & & & & \\ \text { Very False } & \text { Moderately False } & \text { Slightly False } & \text { Slightly True } & \text { Moderately True } & \text { Very True }\end{array}$

31) They have felt deep affection for me.
Very False
Moderately Fals
False Slightly False
$\begin{array}{ccc}4 & 5 & 6 \\ \text { Slightly True } & \text { Moderately True } & \text { Very True }\end{array}$

32) What other people have thought of me have (or would, if they had known) affected the way they have felt toward me.

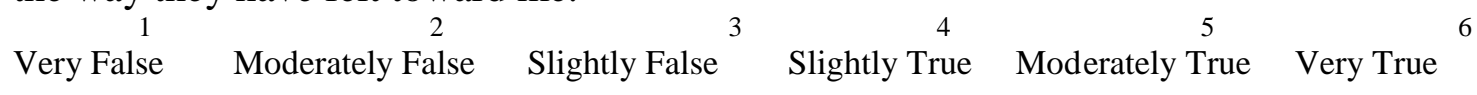




\section{APPENDIX C \\ BODY ACCEPTANCE BY OTHERS SCALE}

Directions for participants: For each item, please circle the answer that best characterizes your experience. Be careful to read each item carefully.

1. I've felt acceptance from $\underline{m y}$ friends regarding my body shape and/or weight.

$\begin{array}{lllll}1 & 2 & 3 & 4 & 5\end{array}$

Never Always

2. I've felt acceptance from my family regarding my body shape and/or weight.
1 2 3 4 5

Never Always

3. I've felt acceptance from people whom I dated regarding my body shape and/or weight.
1
2
3
4
5

Never

Always

4. I've felt acceptance from society regarding my body shape and/or weight.
1
2
3
4
5

Never Always

5. I've felt acceptance from the media regarding my body shape and/or weight.
1
2 3
4
5

Never

Always

6. My friends have sent me the message that my body shape and weight are fine.

$\begin{array}{lllll}1 & 2 & 3 & 4 & 5\end{array}$

Never Always

7. My family have sent me the message that my body shape and weight are fine.

1

2
4

5

Never

Always 
8. People whom I dated have sent me the message that my body shape and weight are fine.

$\begin{array}{llllll}1 & 2 & 3 & 4 & 5\end{array}$

Never

Always

9. Society has sent me the message that my body shape and weight are fine.

$\begin{array}{llllll}1 & 2 & 3 & 4 & 5\end{array}$

Never

Always

10. The media has sent me the message that my body shape and weight are fine. 1

2

3

4

5

Never

Always 


\section{APPENDIX D \\ BODY SURVEILLANCE SUBSCALE OF THE OBJECTIFIED BODY CONSCIOUSNESS}

Directions for participants: For each item, please circle the answer that fits your experience.

1. I rarely think about how I look.

$\begin{array}{llllll}1 & 2 & 3 & 4 & 5 & 6 \\ \text { (Strongly Disagree) } & & & & \text { (Strongly Agree) }\end{array}$

2. I think it is more important that my clothes are comfortable than whether they look good on me.

$\begin{array}{llllll}1 & 2 & 3 & 4 & 5 & 6 \\ \text { (Strongly Disagree) } & & & & \text { (Strongly Agree) }\end{array}$

3. I think more about how my body feels than how my body looks.

$\begin{array}{llllll}1 & 2 & 3 & 4 & 5 & 6 \\ \text { (Strongly Disagree) } & & & & \text { (Strongly Agree) }\end{array}$

4. I rarely compare how I look with how other people look.

$\begin{array}{lcllll}1 & 2 & 3 & 4 & 5 & 6 \\ \text { (Strongly Disagree) } & & & & \text { (Strongly Agree) }\end{array}$

5. During the day, I think about how I look many times.
12
(Strongly Disagree)
3
4
5
6
(Strongly Agree)

6. I often worry about whether the clothes I am wearing make me look good.

$\begin{array}{llllll}1 & 2 & 3 & 4 & 5 & 6 \\ \text { (Strongly Disagree) } & & & & \text { (Strongly Agree) }\end{array}$

7. I rarely worry about how I look to other people.

$\begin{array}{llllll}1 & 2 & 3 & 4 & 5 & 6 \\ \text { (Strongly Disagree) } & & & & \text { (Strongly Agree) }\end{array}$


8. I am more concerned with what my body can do than how it looks.

$1 \quad 2 \quad 3$

(Strongly Disagree)
4

5

6

(Strongly Agree) 


\section{APPENDIX E \\ BODY APPRECIATION SCALE}

Directions for participants: For each item, please indicate whether the question item is true about you never, seldom, sometimes, often, or always.

1. I respect my body.

$\begin{array}{ccccc}1 & 2 & 3 & 4 & 5 \\ \text { Never } & \text { Seldom } & \text { Sometimes } & \text { Often } & \text { Always }\end{array}$

2. I feel good about my body.

$\begin{array}{ccccc}1 & 2 & 3 & 4 & 5 \\ \text { Never } & \text { Seldom } & \text { Sometimes } & \text { Often } & \text { Always }\end{array}$

3. On the whole, I am satisfied with my body.

$\begin{array}{ccccc}1 & 2 & 3 & 4 & 5 \\ \text { Never } & \text { Seldom } & \text { Sometimes } & \text { Often } & \text { Always }\end{array}$

4. Despite its flaws, I accept my body for what it is.

$\begin{array}{ccccc}1 & 2 & 3 & 4 & 5 \\ \text { Never } & \text { Seldom } & \text { Sometimes } & \text { Often } & \text { Always }\end{array}$

5. I feel that my body has at least some good qualities.

$\begin{array}{ccccc}1 & 2 & 3 & 4 & 5 \\ \text { Never } & \text { Seldom } & \text { Sometimes } & \text { Often } & \text { Always }\end{array}$

6. I take a positive attitude towards my body.

$\begin{array}{ccccc}1 & 2 & 3 & 4 & 5 \\ \text { Never } & \text { Seldom } & \text { Sometimes } & \text { Often } & \text { Always }\end{array}$

7. I am attentive to my body's needs.

$\begin{array}{ccccc}1 & 2 & 3 & 4 & 5 \\ \text { Never } & \text { Seldom } & \text { Sometimes } & \text { Often } & \text { Always }\end{array}$


8. My self worth is independent of my body shape or weight.

$\begin{array}{ccccc}1 & 2 & 3 & 4 & 5 \\ \text { Never } & \text { Seldom } & \text { Sometimes } & \text { Often } & \text { Always }\end{array}$

9. I do not focus a lot of energy being concerned with my body shape or weight.

$\begin{array}{ccccc}1 & 2 & 3 & 4 & 5 \\ \text { Never } & \text { Seldom } & \text { Sometimes } & \text { Often } & \text { Always }\end{array}$

10. My feelings toward my body are positive, for the most part.

$\begin{array}{lllll}1 & 2 & 3 & 4 & 5\end{array}$

Never Seldom Sometimes Often Always

11. I engage in healthy behaviors to take care of my body.

$\begin{array}{lllll}1 & 2 & 3 & 4 & 5\end{array}$

Never Seldom Sometimes Often Always

12. I do not allow unrealistically thin images of women presented in the media to affect my attitudes toward my body.

$\begin{array}{ccccc}1 & 2 & 3 & 4 & 5 \\ \text { Never } & \text { Seldom } & \text { Sometimes } & \text { Often } & \text { Always }\end{array}$

13. Despite its imperfections, I still like my body.

$\begin{array}{ccccc}1 & 2 & 3 & 4 & 5 \\ \text { Never } & \text { Seldom } & \text { Sometimes } & \text { Often } & \text { Always }\end{array}$




\section{APPENDIX F \\ INTUITIVE EATING SCALE}

Directions for participants: For each item, please circle the answer that best characterizes your attitudes or behaviors.

1. I try to avoid certain foods high in fat, carbohydrates, or calories.

$\begin{array}{ccccc}1 & 2 & 3 & 4 & 5 \\ \text { Strongly Disagree } & \text { Disagree } & \text { Neutral } & \text { Agree } & \text { Strongly Agree }\end{array}$

2. I stop eating when I feel full (not overstuffed).

$\begin{array}{ccccc}1 & 2 & 3 & 4 & 5 \\ \text { Strongly Disagree } & \text { Disagree } & \text { Neutral } & \text { Agree } & \text { Strongly Agree }\end{array}$

3. I find myself eating when I'm feeling emotional (e.g., anxious, depressed, sad), even when I'm not physically hungry.
Strongly
1
23
4
5
Strongly Disagree Disagree Neutral Agree Strongly Agree

4. If I am craving a certain food, I allow myself to have it.

$\begin{array}{ccccc}1 & 2 & 3 & 4 & 5 \\ \text { Strongly Disagree } & \text { Disagree } & \text { Neutral } & \text { Agree } & \text { Strongly Agree }\end{array}$

5. I follow eating rules or dieting plans that dictate what, when, and/or how much to eat.

$\begin{array}{ccccc}1 & 2 & 3 & 4 & 5 \\ \text { Strongly Disagree } & \text { Disagree } & \text { Neutral } & \text { Agree } & \text { Strongly Agree }\end{array}$

6. I find myself eating when I am bored, even when I'm not physically hungry.

$\begin{array}{ccccc}1 & 2 & 3 & 4 & 5 \\ \text { Strongly Disagree } & \text { Disagree } & \text { Neutral } & \text { Agree } & \text { Strongly Agree }\end{array}$

7. I can tell when I'm slightly full.

$\begin{array}{ccccc}1 & 2 & 3 & 4 & 5 \\ \text { Dly Disagree } & \text { Disagree } & \text { Neutral } & \text { Agree } & \text { Strongly Agree }\end{array}$


8. I can tell when I'm slightly hungry.

$\begin{array}{ccccc}1 & 2 & 3 & 4 & 5 \\ \text { Strongly Disagree } & \text { Disagree } & \text { Neutral } & \text { Agree } & \text { Strongly Agree }\end{array}$

9. I get mad at myself for eating something unhealthy.

$\begin{array}{ccccc}1 & 2 & 3 & 4 & 5 \\ \text { Strongly Disagree } & \text { Disagree } & \text { Neutral } & \text { Agree } & \text { Strongly Agree }\end{array}$

10. I find myself eating when I am lonely, even when I'm not physically hungry.

$\begin{array}{ccccc}1 & 2 & 3 & 4 & 5 \\ \text { Strongly Disagree } & \text { Disagree } & \text { Neutral } & \text { Agree } & \text { Strongly Agree }\end{array}$

11. I trust my body to tell me when to eat.

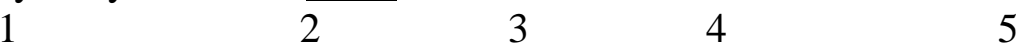

Strongly Disagree Disagree Neutral Agree Strongly Agree

12. I trust my body to tell me what to eat.

$\begin{array}{lcccc}1 & 2 & 3 & 4 & \\ \text { Strongly Disagree } & \text { Disagree } & \text { Neutral } & \text { Agree } & \text { Strongly Agree }\end{array}$

13. I trust my body to tell me how much to eat.

$\begin{array}{ccccc}1 & 2 & 3 & 4 & 5 \\ \text { Strongly Disagree } & \text { Disagree } & \text { Neutral } & \text { Agree } & \text { Strongly Agree }\end{array}$

14. I have forbidden foods that I don't allow myself to eat.

$\begin{array}{ccccc}1 & 2 & 3 & 4 & 5 \\ \text { Strongly Disagree } & \text { Disagree } & \text { Neutral } & \text { Agree } & \text { Strongly Agree }\end{array}$

15. When I'm eating, I can tell when I am getting full.

$\begin{array}{ccccc}1 & 2 & 3 & 4 & 5 \\ \text { Strongly Disagree } & \text { Disagree } & \text { Neutral } & \text { Agree } & \text { Strongly Agree }\end{array}$

16. I use food to help me soothe my negative emotions.

$\begin{array}{ccccc}1 & 2 & 3 & 4 & 5 \\ \text { Strongly Disagree } & \text { Disagree } & \text { Neutral } & \text { Agree } & \text { Strongly Agree }\end{array}$

17. I find myself eating when I am stressed out, even when I'm not physically hungry.

$\begin{array}{lllll}1 & 2 & 3 & 4 & 5\end{array}$

Strongly Disagree Disagree Neutral Agree Strongly Agree 
18. I feel guilty if I eat a certain food that is high in calories, fat, or carbohydrates.
1
2
3
4
5
Strongly Disagree Disagree Neutral Agree Strongly Agree

19. I think of a certain food as "good" or "bad" depending on its nutritional content.

$\begin{array}{ccc}1 & 2 & 3 \\ \text { Strongly Disagree } & \text { Disagree } & \text { Neutral } \\ \text { 20. I don't trust myself around fattening foods. } & 2 & 3\end{array}$ Strongly Disagree Disagree Neutral Agree Strongly Agree

21. I don't keep certain foods in my house/apartment because I think that I may lose control and eat them.

$\begin{array}{ccccc}1 & 2 & 3 & 4 & 5 \\ \text { Strongly Disagree } & \text { Disagree } & \text { Neutral } & \text { Agree } & \text { Strongly Agree }\end{array}$




\section{APPENDIX G \\ ETHNIC IDENTITY SUBSCALE OF THE MULTIGROUP ETHNIC IDENTITY MEASURE}

In this country, people come from a lot of different cultures and there are many different words to describe the different backgrounds of ethnic groups that people come from. Some examples of the names of ethnic groups are Mexican-American, Hispanic, Black, Asian-American, American Indian, Anglo-American, and White. Every person is born into an ethnic group, or sometimes two ethnic groups, but people differ on how important their ethnicity is to them, how they feel about it, and how much their behavior is affected by it. These questions are about your ethnicity or your ethnic group and how you feel about it or react to it.

Please fill in:

In terms of ethnic group, I consider myself to be

Use the number given below to indicate how much you agree or disagree with each statement

1. I have spent time trying to find out more about my own ethnic group, such as its history, traditions, and customs.

$\begin{array}{cccc}1 & 2 & 3 & 4 \\ \text { Strongly Disagree } & \text { Somewhat Disagree } & \text { Agree } & \text { Strongly Agree }\end{array}$

2. I am active in organizations of social groups that include mostly members of my own ethnic group.

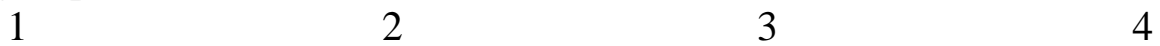

Strongly Disagree Somewhat Disagree Agree Strongly Agree

3. I have a clear sense of my ethnic background and what it means to me.

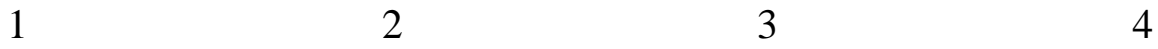

Strongly Disagree Somewhat Disagree Agree Strongly Agree 
4. I think a lot about how my life will be affected by my ethnic group membership.

$1 \quad 2 \quad 3 \quad 4$

Strongly Disagree Somewhat Disagree Agree Strongly Agree

5. I am happy that I am a member of the group I belong to.

1 2 304

Strongly Disagree Somewhat Disagree Agree Strongly Agree

6. I am not very clear about the role of my ethnicity in my life.

$1 \quad 2 \quad 3 \quad 4$

Strongly Disagree Somewhat Disagree Agree Strongly Agree

7. I really have not spent much time trying to learn more about the culture and history of my ethnic group.

$\begin{array}{cccc}1 & 2 & 3 & 4 \\ \text { Strongly Disagree } & \text { Somewhat Disagree } & \text { Agree } & \text { Strongly Agree }\end{array}$

8. I have a strong sense of belonging to my own ethnic group.

12324

Strongly Disagree Somewhat Disagree Agree Strongly Agree

9. I understand pretty well what my ethnic group membership means to me, in terms of how to relate to my own group and other groups.

$\begin{array}{llll}1 & 2 & 3 & 4\end{array}$

Strongly Disagree Somewhat Disagree Agree Strongly Agree

10. In order to learn more about my ethnic background, I have often talked to other people about my ethnic group.

$\begin{array}{cccc}1 & 2 & 3 & 4 \\ \text { Strongly Disagree } & \text { Somewhat Disagree } & \text { Agree } & \text { Strongly Agree }\end{array}$

11. I have a lot of pride in my ethnic group and its accomplishments.

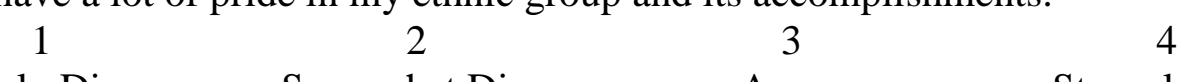

Strongly Disagree Somewhat Disagree Agree Strongly Agree

12. I participate in cultural practices of my own group, such as special food, music, or customs.

$1 \quad 2 \quad 3 \quad 4$

Strongly Disagree Somewhat Disagree Agree Strongly Agree 
13. I feel a strong attachment toward my own ethnic group.

1

Strongly Disagree Somewhat Disagree

14. I feel good about my cultural or ethnic background. 1

Strongly Disagree Somewhat Disagree
3

Agree

3

Agree
4

Strongly Agree

4

Strongly Agree 


\section{APPENDIX H}

\section{INTERNALIZATION AFROCENTRICITY SUBSCALE OF THE CROSS RACIAL IDENTITY SCALE}

Instructions: Read each item and indicate to what degree it reflects your own thoughts and feelings, using the 7-point scale below. There are no right or wrong answers. Base your responses on your opinion at the present time.

1. I see and think about things from an Afrocentric perspective.

$\begin{array}{lcccccc}1 & 2 & 3 & 4 & 5 & 6 & 7 \\ \begin{array}{llll}\text { Strongly } \\ \text { Disagree }\end{array} & \text { Disagree } & \begin{array}{l}\text { Somewhat } \\ \text { Disagree }\end{array} & \begin{array}{c}\text { Neither } \\ \text { Disagree or Agree }\end{array} & \begin{array}{c}\text { Somewhat } \\ \text { Agree }\end{array} & \text { Agree } & \text { Strongly } \\ \text { Agree }\end{array}$

2. I believe that only those Black people who accept an Afrocentric perspective can truly solve the race problem in America.

$\begin{array}{lcccccc}1 & 2 & 3 & 4 & 5 & 6 & 7 \\ \begin{array}{llll}\text { Strongly } \\ \text { Disagree }\end{array} & \text { Disagree } & \text { Somewhat } & \text { Neither } & \text { Somewhat } & \text { Agree } & \text { Strongly } \\ \text { Disagree } & & \text { Disagree or Agree } & \text { Agree } & & \text { Agree }\end{array}$

3. Black people cannot truly be free until our daily lives are guided by Afrocentric values and principles.

$\begin{array}{lcccccc}1 & 2 & 3 & 4 & 5 & 6 & 7 \\ \text { Strongly } & \text { Disagree } & \text { Somewhat } & \text { Neither } & \text { Somewhat } & \text { Agree } & \text { Strongly } \\ \text { Disagree } & & \text { Disagree } & \text { Disagree or Agree } & \text { Agree } & & \text { Agree }\end{array}$

4. I respect the ideas that other Black people hold, but I believe that the best way to solve our problems is to think Afrocentrically.

$\begin{array}{lcccccc}1 & 2 & 3 & 4 & 5 & 6 & 7 \\ \begin{array}{llll}\text { Strongly } \\ \text { Disagree }\end{array} & \text { Disagree } & \text { Somewhat } & \text { Neither } & \text { Somewhat } & \text { Agree } & \text { Strongly } \\ \text { Disagree } & & \text { Disagree or Agree } & \text { Agree } & & \text { Agree }\end{array}$


5. Black people will never be free until we embrace an Afrocentric perspective.

$\begin{array}{lcccccc}1 & 2 & 3 & 4 & 5 & 6 & 7 \\ \begin{array}{llll}\text { Strongly } \\ \text { Disagree }\end{array} & \text { Disagree } & \begin{array}{l}\text { Somewhat } \\ \text { Disagree }\end{array} & \begin{array}{c}\text { Neither } \\ \text { Disagree or Agree }\end{array} & \begin{array}{c}\text { Somewhat } \\ \text { Agree }\end{array} & \text { Agree } & \text { Strongly } \\ \text { Agree }\end{array}$




\section{APPENDIX I \\ RESULTS WITH OUTLIERS EXCLUDED FROM DATA ANALYSIS}

Table 5

Means, Standard Deviations, Skewness, and Kurtosis for Measures With Responses of 126 African American College Women

\begin{tabular}{|c|c|c|c|c|c|c|c|c|}
\hline & Minimum & Maximum & $M$ & $S D$ & Skewness & $S E$ & Kurtosis & $S E$ \\
\hline $\begin{array}{l}\text { Barrett- } \\
\text { Lennard } \\
\text { Relationship } \\
\text { Inventory }\end{array}$ & 2.75 & 5.56 & 4.37 & .641 & -.322 & .216 & -.639 & .428 \\
\hline $\begin{array}{l}\text { Body } \\
\text { Acceptance } \\
\text { by Others } \\
\text { Scale }\end{array}$ & 1.50 & 5.00 & 3.76 & .746 & -.275 & .216 & -.143 & .428 \\
\hline $\begin{array}{l}\text { Body } \\
\text { Surveillance } \\
\text { subscale of } \\
\text { OBC Scale }\end{array}$ & 1.50 & 5.50 & 3.54 & .809 & -.090 & .216 & -.080 & .428 \\
\hline $\begin{array}{l}\text { Body } \\
\text { Appreciation } \\
\text { Scale }\end{array}$ & 2.38 & 5.00 & 3.94 & .548 & -.307 & .216 & -.320 & .428 \\
\hline $\begin{array}{l}\text { Intuitive } \\
\text { Eating Scale }\end{array}$ & 1.67 & 4.76 & 3.35 & .543 & -.074 & .216 & -.121 & .428 \\
\hline $\begin{array}{l}\text { Ethnic } \\
\text { Identity } \\
\text { subscale of } \\
\text { MEIM }\end{array}$ & 2.43 & 4.00 & 3.34 & .413 & -.444 & .216 & -.743 & .428 \\
\hline $\begin{array}{l}\text { Internalization } \\
\text { Afrocentricity } \\
\text { subscale of } \\
\text { CRIS }\end{array}$ & 1.00 & 6.40 & 3.41 & 1.15 & .098 & .216 & -.034 & .428 \\
\hline
\end{tabular}


Table 6

Correlations Among the Measures With Responses of 126 African American College Women

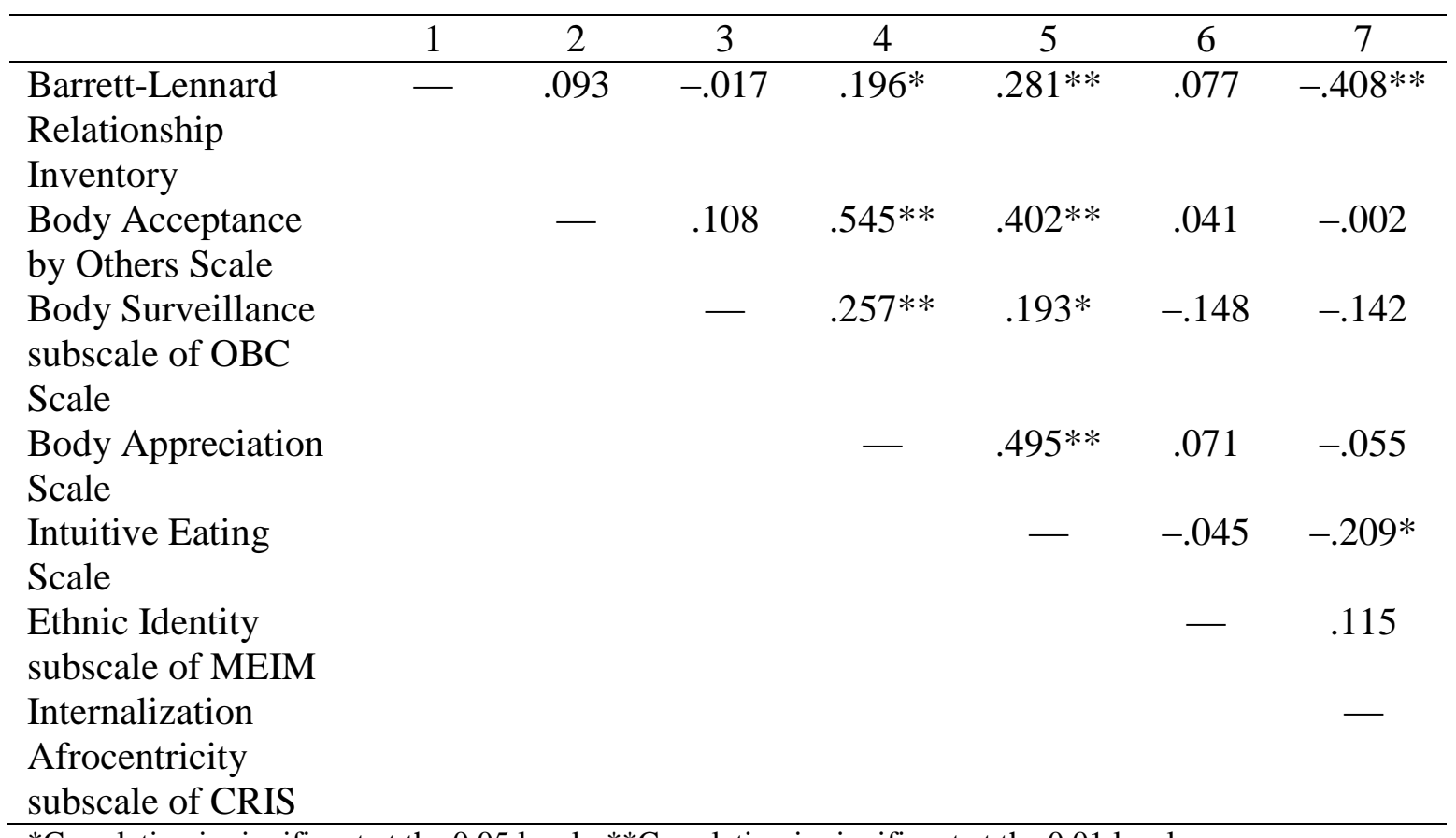

*Correlation is significant at the 0.05 level. **Correlation is significant at the 0.01 level. 
Table 7

Mean, Standard Deviation, and Correlations Among Items on the Body Appreciation Scale With Responses of 126 African American College Women

\begin{tabular}{|c|c|c|c|c|c|c|c|c|c|c|c|c|c|}
\hline & 1 & 2 & 3 & 4 & 5 & 6 & 7 & 8 & 9 & 10 & 11 & 12 & 13 \\
\hline Item 1 & - & .498 & .315 & .472 & .340 & .450 & .569 & .184 & .053 & .423 & .341 & .074 & .384 \\
\hline Item 2 & & - & .650 & .550 & .304 & .547 & .410 & .125 & .028 & .571 & .337 & .093 & .486 \\
\hline Item 3 & & & - & .639 & .328 & .561 & .290 & .115 & .029 & .662 & .369 & .165 & .529 \\
\hline Item 5 & & & & & - & .552 & .352 & .062 & -.081 & .448 & .238 & .086 & .536 \\
\hline Item 6 & & & & & & - & .488 & .275 & .011 & .704 & .438 & .099 & 639 \\
\hline Item 7 & & & & & & & - & .187 & -.053 & .409 & .392 & .028 & .364 \\
\hline Item 10 & & & & & & & & & & - & .438 & .079 & .691 \\
\hline Item 11 & & & & & & & & & & & - & .049 & .234 \\
\hline Item 12 & & & & & & & & & & & & - & .117 \\
\hline Item 13 & & & & & & & & & & & & & - \\
\hline$M$ & 4.44 & 3.79 & 3.79 & 4.13 & 4.47 & 4.09 & 4.04 & 3.79 & 3.17 & 3.96 & 3.70 & 3.49 & 4.41 \\
\hline
\end{tabular}




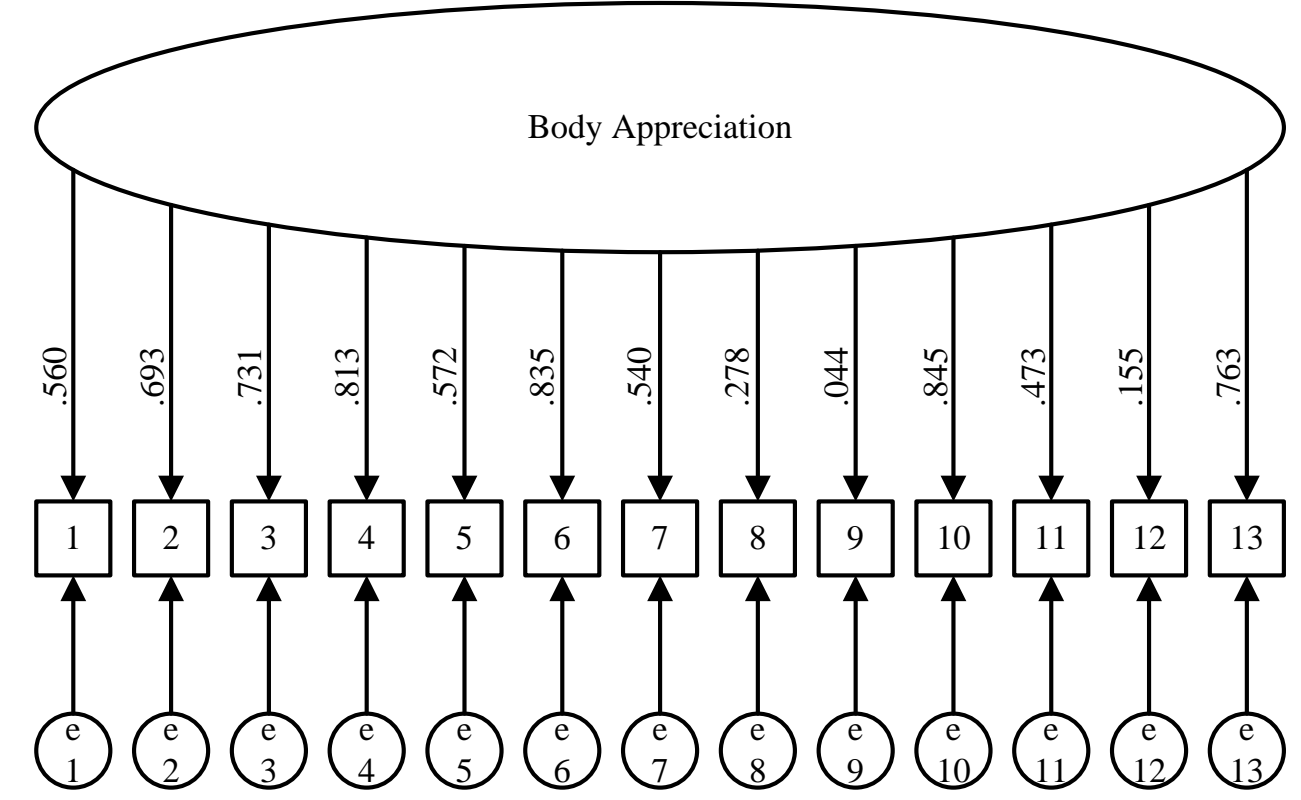

Figure 6. One-factor model of Body Appreciation Scale with factor loadings depicted from data obtained from 126 African American college women. 


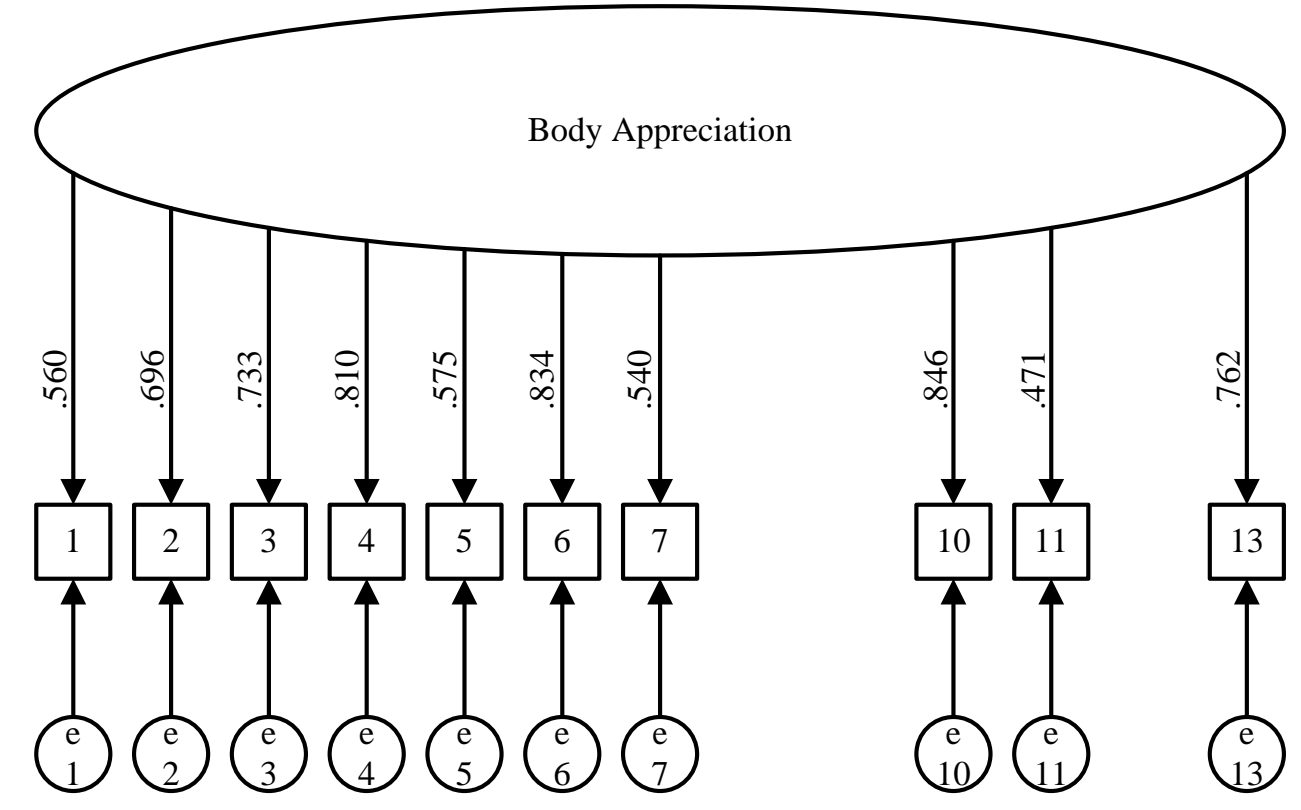

Figure 7. One-factor model of Body Appreciation Scale with Items 8, 9, and 12 deleted with factor loadings depicted from data obtained from 126 African American college women. 


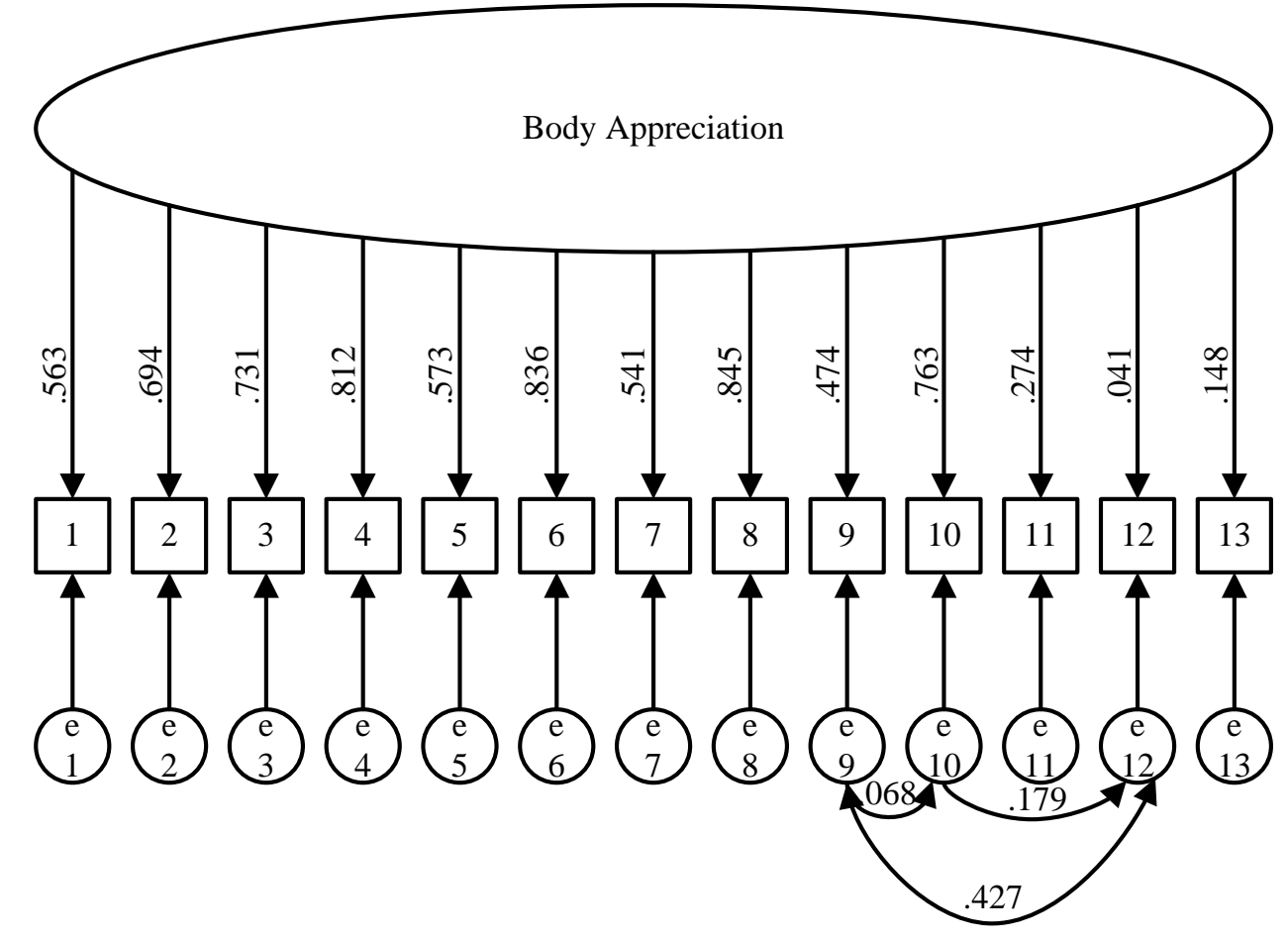

Figure 8. One-factor model of Body Appreciation Scale with error terms associated with Items 8,9 , and 12 allowed to covary and factor loadings depicted from data obtained from 126 African American college women. 


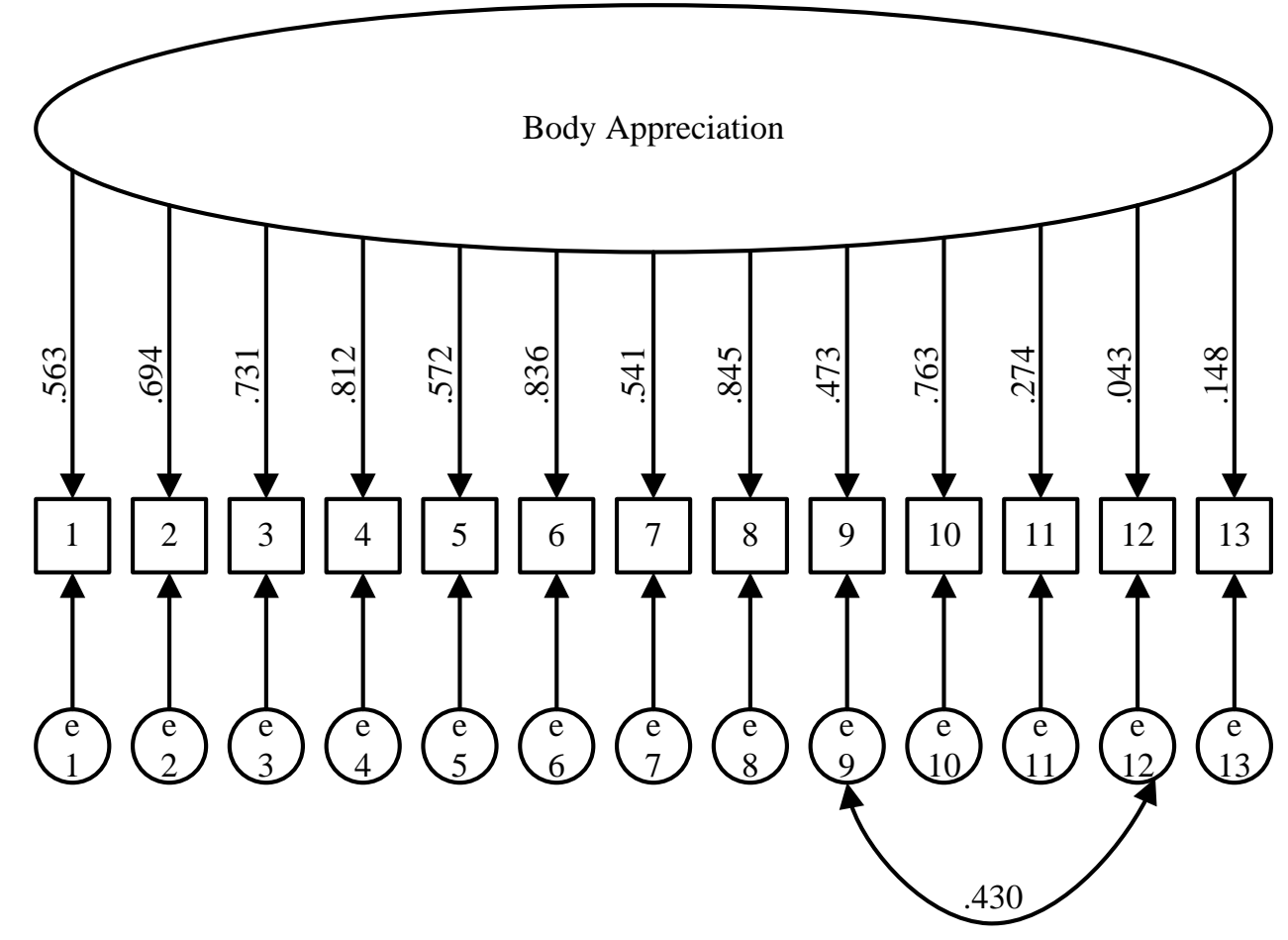

Figure 9. One-factor model of Body Appreciation Scale with error terms associated with Items 8 and 12 allowed to covary and factor loadings depicted from data obtained from 126 African American college women. 
Table 8

Goodness-of-Fit Indices for One-Factor Confirmatory Factor Analyses With Responses From 126 African American College Women on the Body Appreciation Scale

\begin{tabular}{lcccccccccc}
\hline Model & $\chi^{2}$ & $d f$ & $\chi^{2} / d f$ & $p$ & CFI & TLI & RMSR & RMSEA & $90 \%$ CI \\
\hline $\begin{array}{l}13 \text { items } \\
\text { uncorr } \\
\text { error terms }\end{array}$ & 125.523 & 65 & 1.931 & $<.001$ & .905 & .887 & .068 & .086 & .063 & .109 \\
11 items & 92.188 & 35 & 2.634 & $<.001$ & .909 & .883 & .046 & .114 & .086 & .143 \\
uncorrerror \\
terms
\end{tabular}


Table 9

Factor Loadings for the One-Factor Confirmatory Factor Analyses With Responses From 126 African American College Women on the Body Appreciation Scale

\begin{tabular}{lcccc}
\hline 13 items uncorr & 11 items uncorr & $\begin{array}{c}13 \text { items error } \\
\text { terms on } 3 \text { corr }\end{array}$ & $\begin{array}{c}13 \text { items error } \\
\text { terms on } 2 \text { corr }\end{array}$ \\
\hline 1 & .560 & .560 & .563 & .563 \\
2 & .693 & .696 & .694 & .694 \\
3 & .731 & .733 & .731 & .731 \\
4 & .813 & .810 & .812 & .812 \\
5 & .572 & .575 & .573 & .572 \\
6 & .835 & .834 & .836 & .836 \\
7 & .540 & .540 & .541 & .541 \\
8 & .278 & & .845 & .845 \\
9 & .044 & & .474 & .473 \\
10 & .845 & .846 & .763 & .763 \\
11 & .473 & .471 & .274 & .274 \\
12 & .155 & & .041 & .043 \\
13 & .763 & .762 & .148 & .148 \\
\hline
\end{tabular}


Table 10

Model Comparison for One-Factor Confirmatory Factor Analyses With Responses From 126 African American College Women on the Body Appreciation Scale

\begin{tabular}{lccccc}
\hline & $\chi^{2}$ & $d f$ & $\Delta \chi^{2}$ & $\Delta d f$ & $p$ \\
\hline 13 items uncorr & 125.523 & 65 & 10.40 & 3 & .015 \\
13 items error terms on 3 corr & 115.121 & 62 & & & \\
13 items error terms on 2 corr & 117.152 & 64 & 8.371 & 1 & $<.05$ \\
\cline { 3 - 6 } & & & 2.031 & 2 & .362 \\
\hline
\end{tabular}

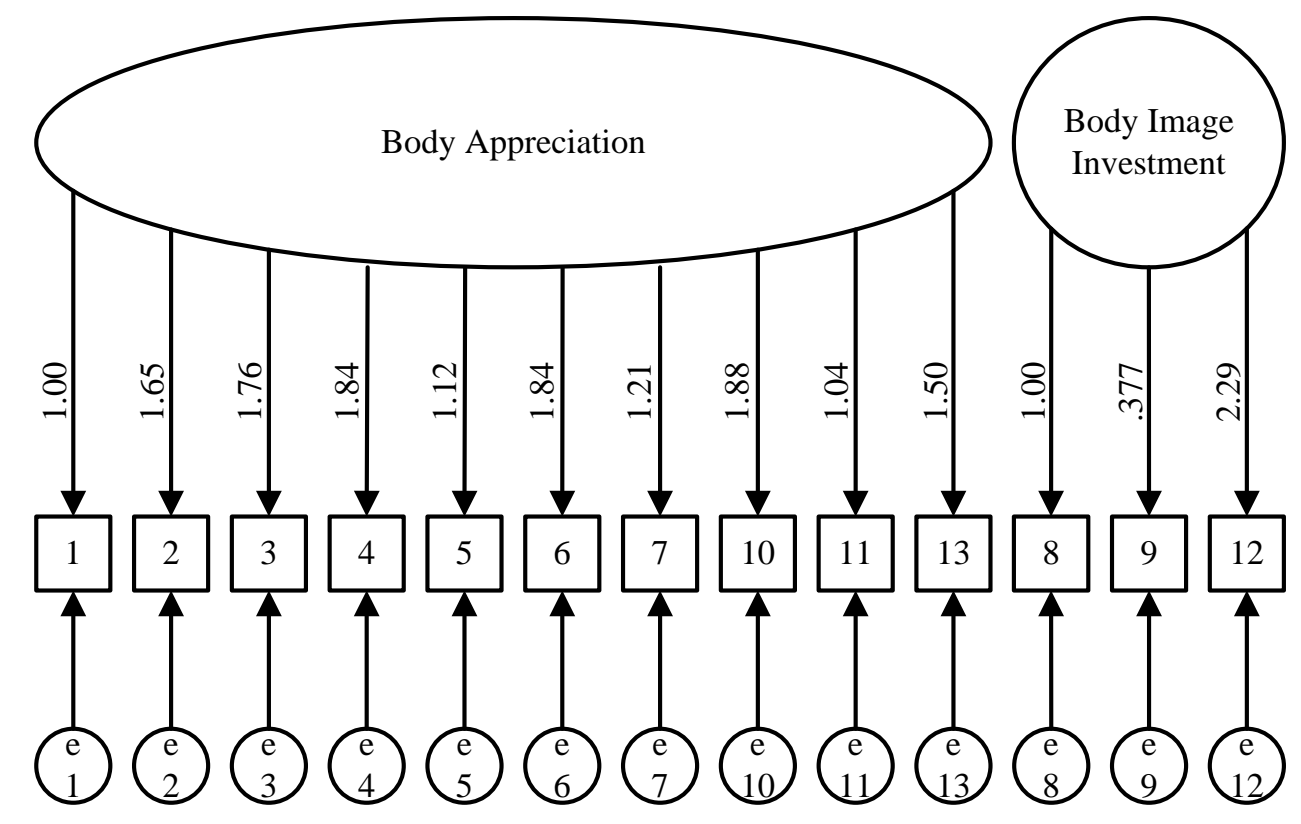

Figure 10. Two-factor model of body appreciation with body appreciation and body image investment uncorrelated from data obtained from 126 African American college women. 


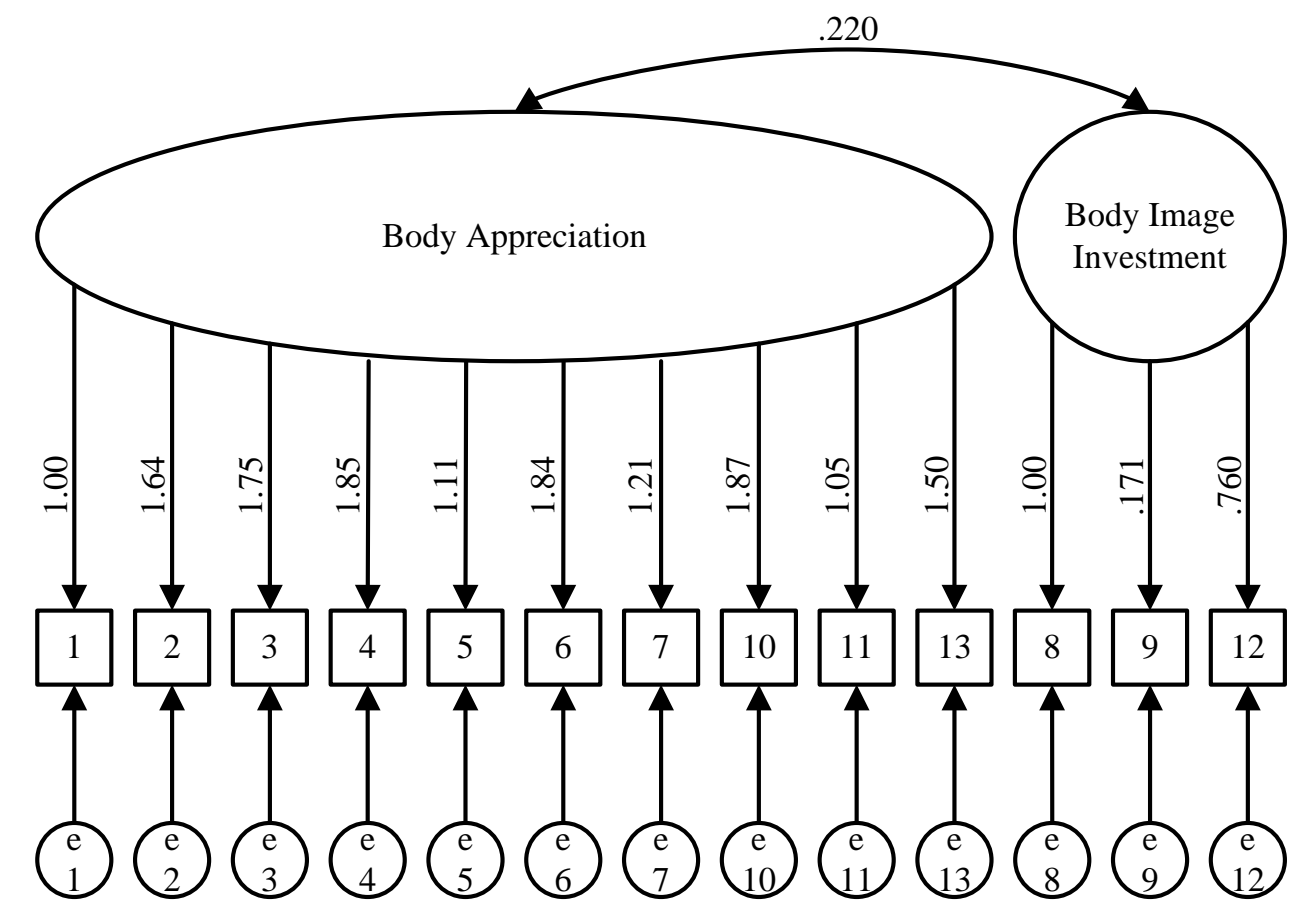

Figure 11. Two-factor model of body appreciation with body appreciation and body image investment correlated from data obtained from 126 African American college women. 
Table 11

Factor Loadings for the Two-Factor Confirmatory Factor Analyses With Responses From 126 African American College Women on the Body Appreciation Scale

\begin{tabular}{lcc}
\hline & 13 items, uncorr factors & 13 items, corr factors \\
\hline 1 & 1.00 & 1.00 \\
2 & 1.65 & 1.64 \\
3 & 1.76 & 1.75 \\
4 & 1.84 & 1.85 \\
5 & 1.12 & 1.11 \\
6 & 1.84 & 1.84 \\
7 & 1.21 & 1.21 \\
8 & 1.00 & 1.00 \\
9 & .377 & .171 \\
10 & 1.88 & 1.87 \\
11 & 1.04 & 1.05 \\
12 & 2.29 & .760 \\
13 & 1.50 & 1.50 \\
\hline
\end{tabular}

Table 12

Goodness-of-Fit Indices for Two-Factor Confirmatory Factor Analyses With Responses From 126 African American College Women on the Body Appreciation Scale

\begin{tabular}{|c|c|c|c|c|c|c|c|c|c|}
\hline Model & $\chi^{2}$ & $d f$ & $\chi^{2} / d f$ & $p$ & CFI & TLI & RMSR & RMSEA & $90 \% \mathrm{CI}$ \\
\hline $\begin{array}{l}13 \text { items } \\
\text { uncorrelated } \\
\text { factors }\end{array}$ & 124.800 & 65 & 1.920 & $<.001$ & .907 & .888 & .091 & .086 & . $1007 \quad$. \\
\hline $\begin{array}{l}13 \text { items } \\
\text { correlated } \\
\text { factors }\end{array}$ & 116.137 & 64 & 1.815 & $<.001$ & .919 & .901 & .049 & .081 & 104 \\
\hline
\end{tabular}


Table 13

Goodness-of-Fit Indices for Path Analyses With Responses From 126 African American College Women

\begin{tabular}{|c|c|c|c|c|c|c|c|c|c|c|c|}
\hline Model & $X^{2}$ & $d f$ & $\chi^{2} / d f$ & $p$ & CFI & TLI & RMSR & RMSEA & \multicolumn{2}{|c|}{$90 \% \mathrm{CI}$} & AIC \\
\hline Original & 15.63 & 4 & 3.91 & .004 & .878 & .695 & .030 & .153 & .078 & .236 & 37.6 \\
\hline Culturally & 44.4 & 8 & 5.55 & .000 & .695 & .428 & .083 & .191 & .138 & .247 & 70.3 \\
\hline Relevant & & & & & & & & & & & \\
\hline Alt- & & & & & & & & & & & \\
\hline Racial & & & & & & & & & & & \\
\hline Identity & & & & & & & & & & & \\
\hline Culturally & 21.4 & 8 & 2.68 & .006 & .861 & .739 & .028 & .116 & .058 & .177 & 47.44 \\
\hline Relevant & & & & & & & & & & & \\
\hline Alt- & & & & & & & & & & & \\
\hline Ethnic & & & & & & & & & & & \\
\hline Identity & & & & & & & & & & & \\
\hline
\end{tabular}




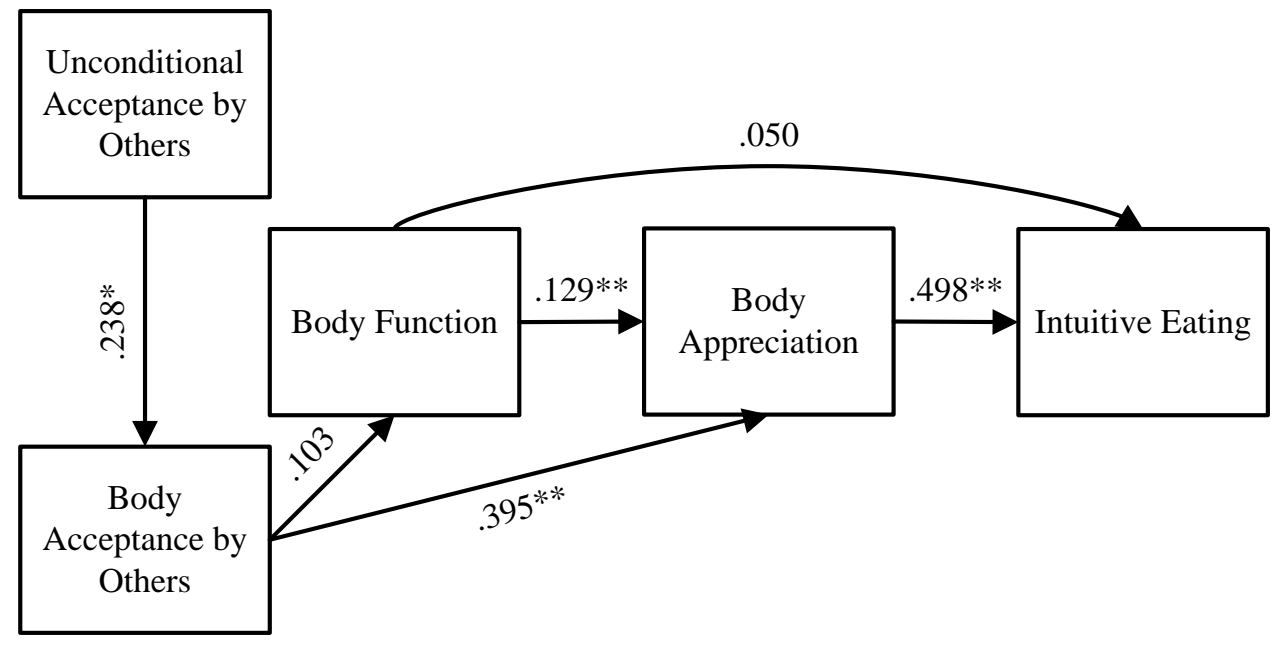

Figure 12. Original model of intuitive eating with path coefficients depicted from data obtained from 126 African American college women. *Correlation is significant at the 0.05 level. $* *$ Correlation is significant at the 0.01 level. 




Figure 13. Culturally relevant alternative model of intuitive eating with racial identity and path coefficients depicted from data obtained from 126 African American college women. *Correlation is significant at the 0.05 level. **Correlation is significant at the 0.01 level. 


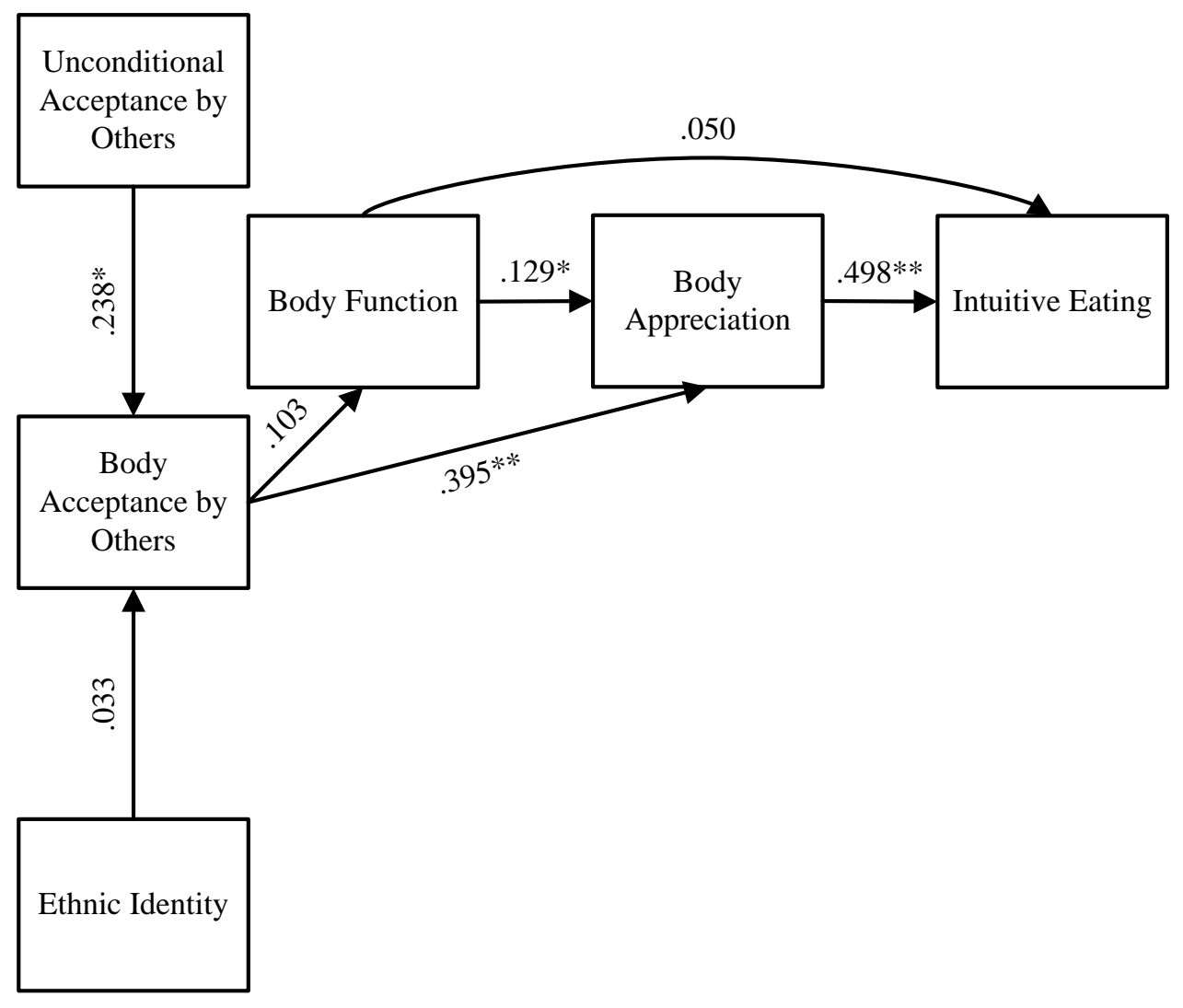

Figure 14. Culturally relevant alternative model of intuitive eating with ethnic identity and path coefficients depicted from data obtained from 126 African American college women. *Correlation is significant at the 0.05 level. **Correlation is significant at the 0.01 level. 


\section{APPENDIX J}

\section{TABLES AND FIGURES DEPICTING CONFIRMATORY FACTOR ANALYSIS OF BODY APPRECIATION SCALE}

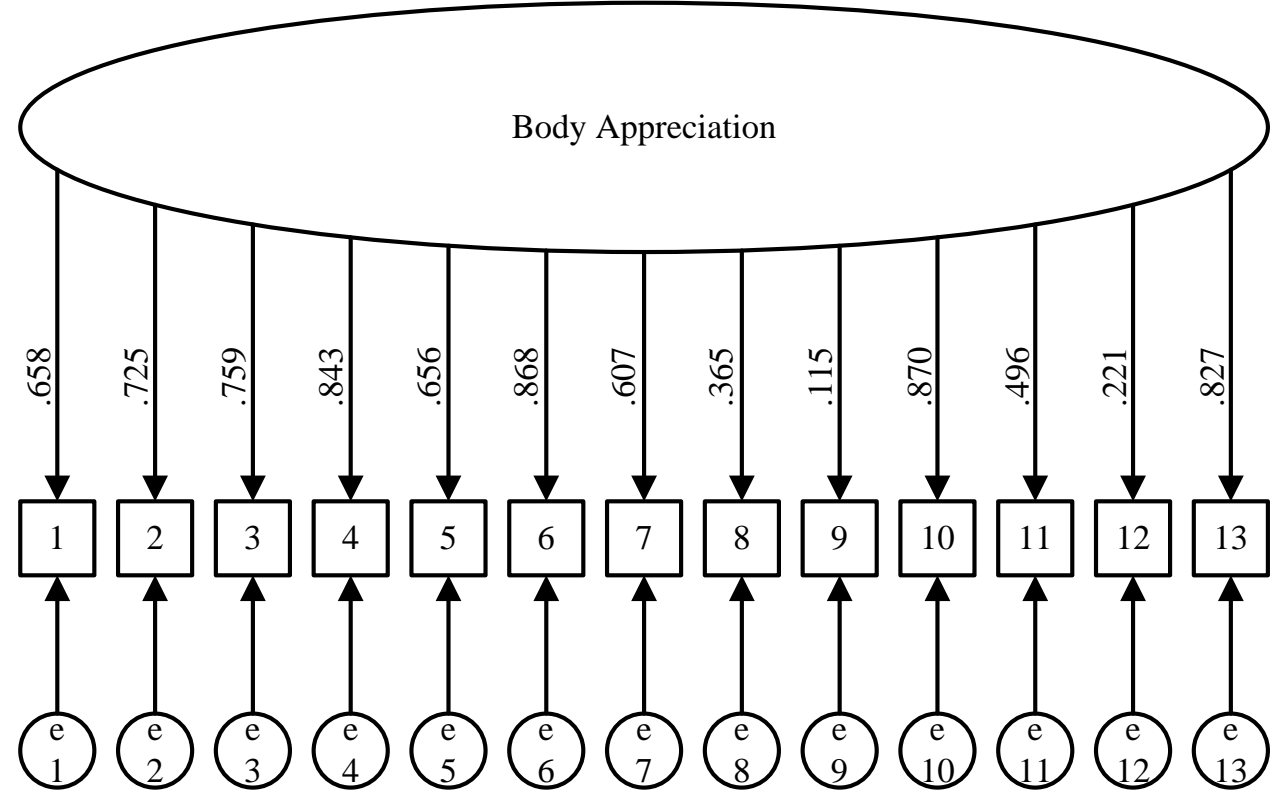

Figure 15. One-factor model of Body Appreciation Scale with factor loadings depicted from data obtained from 130 African American college women. 


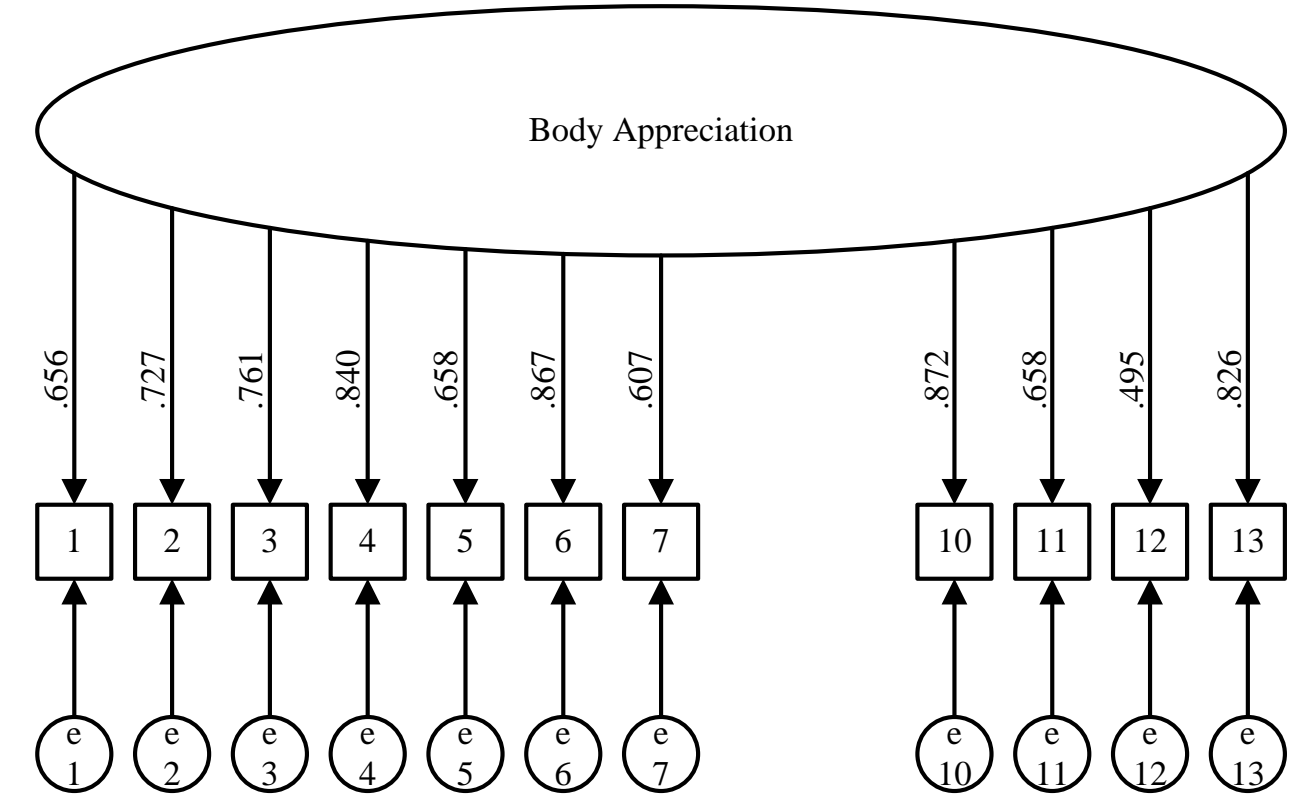

Figure 16. One-factor model of Body Appreciation Scale with Items 8, 9, and 12 deleted with factor loadings depicted from data obtained from 130 African American college women. 


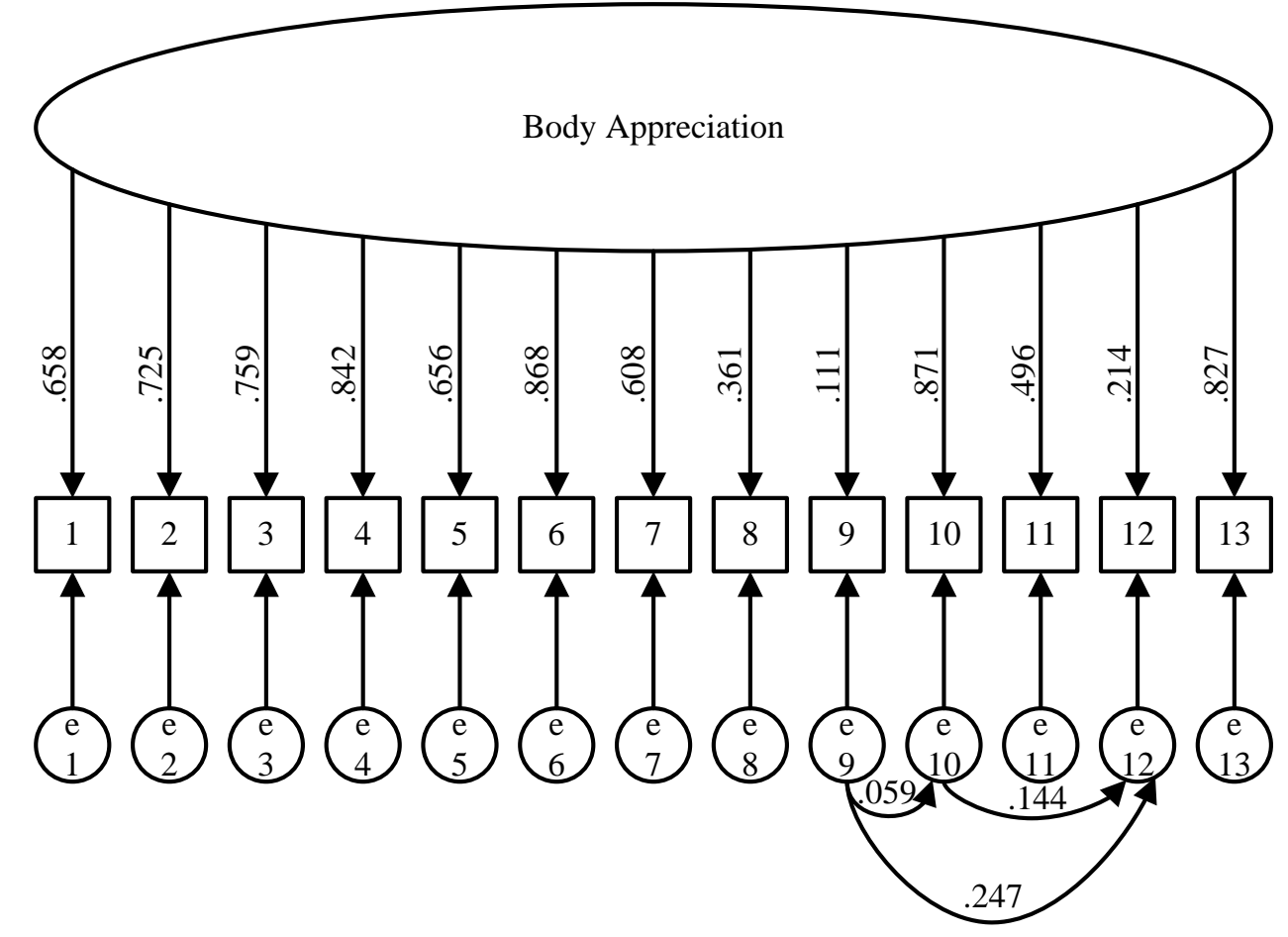

Figure 17. One-factor model of Body Appreciation Scale with error terms associated with Items 8, 9, and 12 allowed to covary and factor loadings depicted from data obtained from 130 African American college women. 


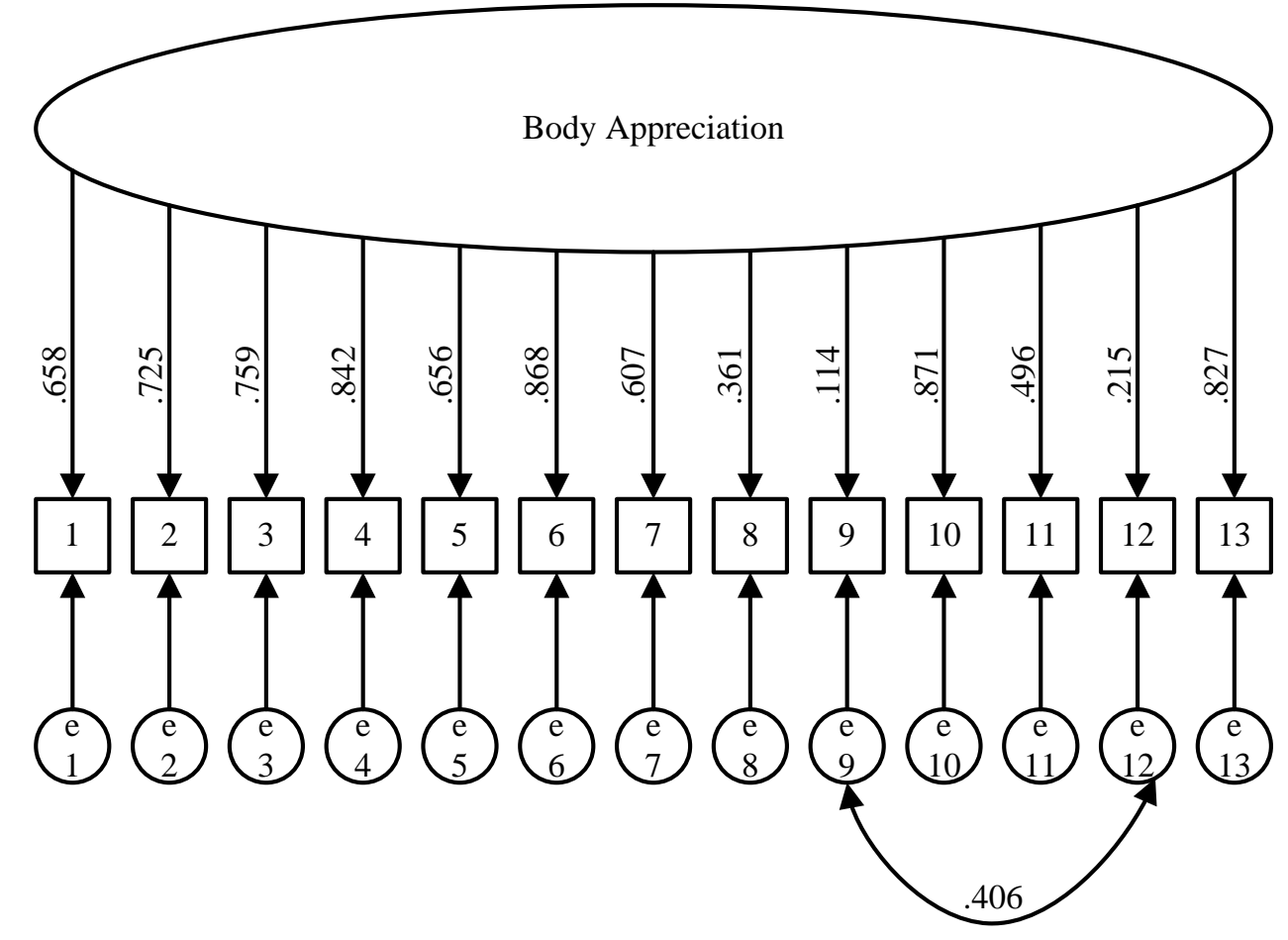

Figure 18. One-factor model of Body Appreciation Scale with error terms associated with Items 8 and 12 allowed to covary and factor loadings depicted from data obtained from 130 African American college women. 
Table 14

Goodness-of-Fit Indices for One-Factor Confirmatory Factor Analyses for Responses From 130 African American College Women on the Body Appreciation Scale

\begin{tabular}{|c|c|c|c|c|c|c|c|c|c|c|}
\hline Model & $\chi^{2}$ & $d f$ & $\chi^{2} / d f$ & $p$ & CFI & TLI & RMSR & RMSEA & \multicolumn{2}{|c|}{$90 \% \mathrm{CI}$} \\
\hline $\begin{array}{l}13 \text { items } \\
\text { uncorr } \\
\text { error } \\
\text { terms }\end{array}$ & 128.698 & 65 & 1.970 & $<.001$ & .924 & .908 & .067 & .087 & .065 & .109 \\
\hline $\begin{array}{l}11 \text { items } \\
\text { uncorr } \\
\text { error } \\
\text { terms }\end{array}$ & 96.980 & 35 & 2.770 & $<.001$ & .923 & .902 & .047 & .117 & .090 & .145 \\
\hline $\begin{array}{l}13 \text { items } \\
\text { error } \\
\text { terms on } 3 \\
\text { items } \\
\text { allowed to } \\
\text { corr }\end{array}$ & 117.936 & 62 & 1.902 & $<.001$ & .933 & .915 & .046 & .084 & .060 & .106 \\
\hline $\begin{array}{l}13 \text { items } \\
\text { error } \\
\text { terms on } 2 \\
\text { items } \\
\text { allowed to } \\
\text { corr }\end{array}$ & 120.714 & 64 & 1.886 & $<.001$ & .932 & .917 & .052 & .083 & .060 & .105 \\
\hline
\end{tabular}


Table 15

Factor Loadings for the One-Factor Confirmatory Factor Analyses for Responses From 130 African American College Women on the Body Appreciation Scale

\begin{tabular}{ccccc}
\hline & 13 items uncorr & 11 items uncorr & $\begin{array}{c}13 \text { items error } \\
\text { terms on } 3 \text { corr }\end{array}$ & $\begin{array}{c}13 \text { items error } \\
\text { terms on } 2 \text { corr }\end{array}$ \\
\hline 1 & .658 & .656 & .658 & .658 \\
2 & .725 & .727 & .725 & .725 \\
3 & .759 & .761 & .759 & .759 \\
4 & .843 & .840 & .842 & .842 \\
5 & .656 & .658 & .656 & .656 \\
6 & .868 & .867 & .868 & .868 \\
7 & .607 & .607 & .608 & .607 \\
8 & .365 & & .361 & .361 \\
9 & .115 & & .111 & .114 \\
10 & .870 & .872 & .871 & .871 \\
11 & .496 & .495 & .496 & .496 \\
12 & .221 & & .214 & .215 \\
13 & .827 & .826 & .827 & .827 \\
\hline
\end{tabular}

Table 16

Model Comparisons for One-Factor Confirmatory Factor Analyses of Responses From 130 African American College Women on the Body Appreciation Scale

\begin{tabular}{lccccc}
\hline & $\chi^{2}$ & $d f$ & $\Delta \chi^{2}$ & $\Delta d f$ & $p$ \\
\hline 13 items uncorr & 128.698 & 65 & 10.76 & 3 & $<.05$ \\
13 items error & 117.936 & 62 & & & \\
$\begin{array}{l}\text { terms on 3 corr } \\
13 \text { items error }\end{array}$ & 120.714 & 64 & 8 & 1 & $<.001$ \\
terms on 2 corr & & & & & .250 \\
\hline
\end{tabular}




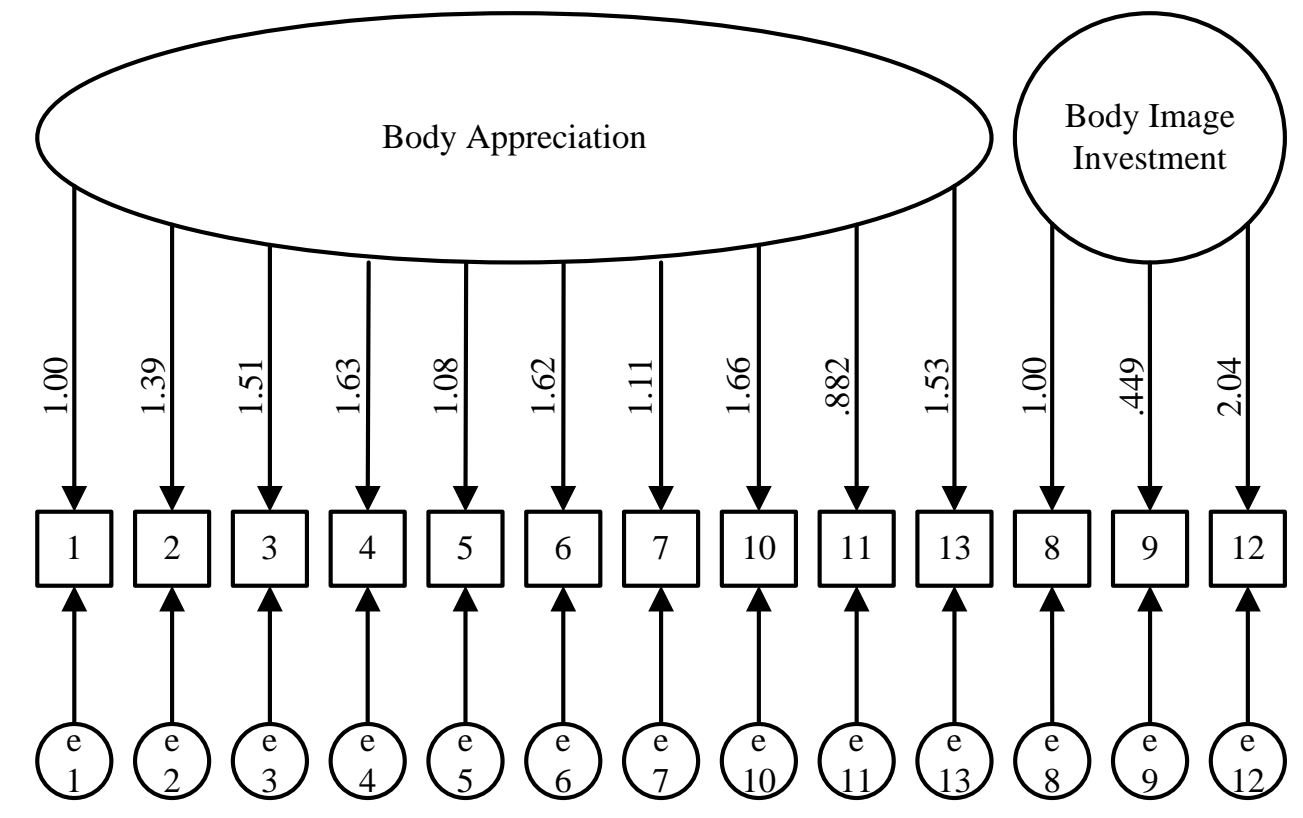

Figure 19. Two-factor model of body appreciation with body appreciation and body image investment uncorrelated from data obtained from 130 African American college women. 


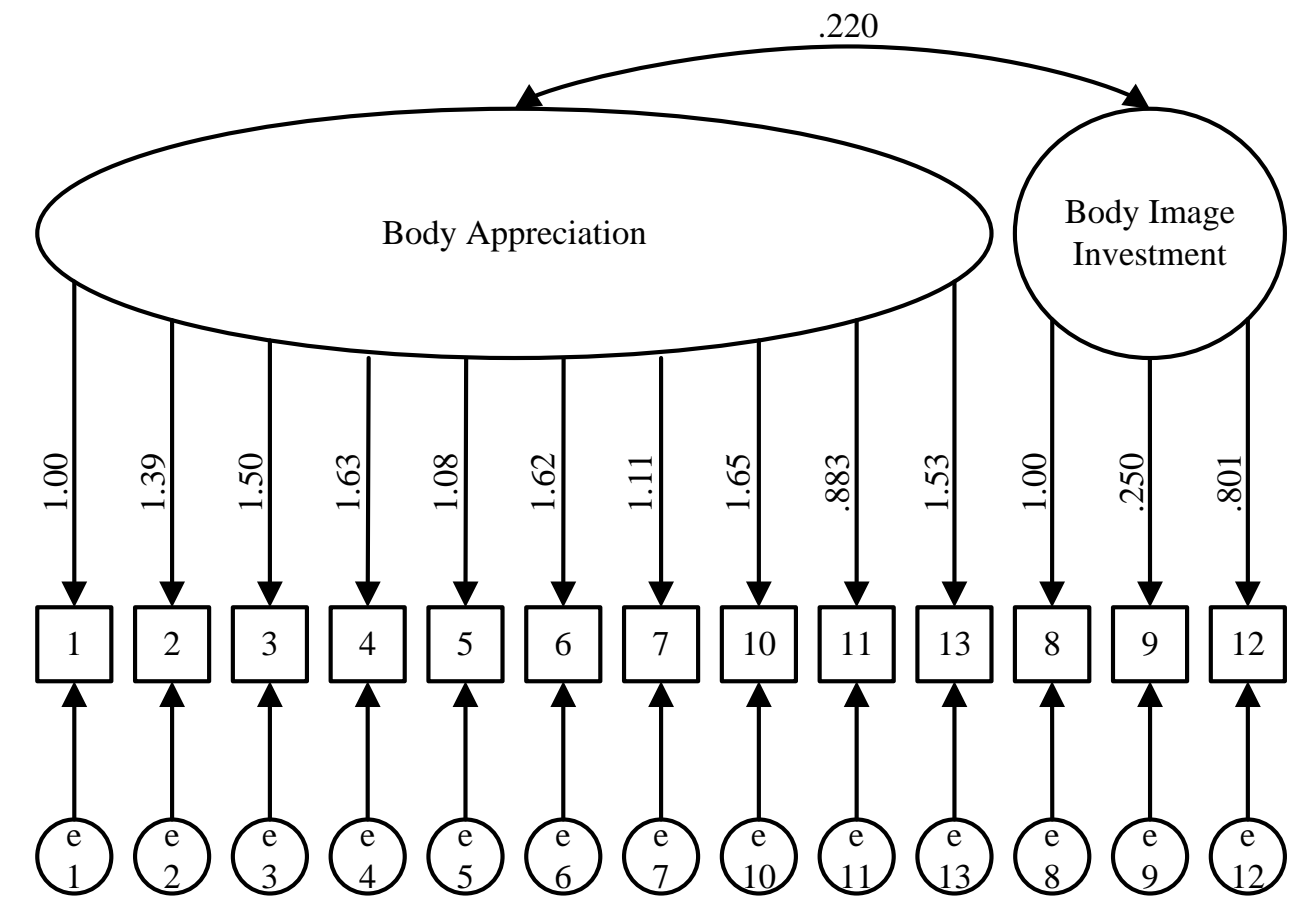

Figure 20. Two-factor model of body appreciation with body appreciation and body image investment correlated for data obtained from 130 African American college women. 
Table 17

Factor Loadings for the Two-Factor Confirmatory Factor Analyses of Responses From 130 African American College Women on the Body Appreciation Scale

\begin{tabular}{lcc}
\hline & 13 items, uncorr factors & 13 items, corr factors \\
\hline 1 & 1.00 & 1.00 \\
2 & 1.39 & 1.39 \\
3 & 1.51 & 1.50 \\
4 & 1.63 & 1.63 \\
5 & 1.08 & 1.08 \\
6 & 1.62 & 1.62 \\
7 & 1.11 & 1.11 \\
8 & 1.00 & 1.00 \\
9 & .449 & .250 \\
10 & 1.66 & 1.65 \\
11 & .882 & .883 \\
12 & 2.03 & .801 \\
13 & 1.53 & 1.53 \\
\hline
\end{tabular}

Table 18

Goodness-of-Fit Indices for Two-Factor Confirmatory Factor Analyses of Responses From 130 African American College Women on the Body Appreciation Scale

\begin{tabular}{lcccccccccc}
\hline Model & $\chi^{2}$ & $d f$ & $\chi^{2} / d f$ & $p$ & CFI & TLI & RMSR & RMSEA & $90 \%$ CI \\
$\begin{array}{c}\text { 13 items, } \\
\text { uncorr }\end{array}$ & 136.99 & 65 & 2.108 & $<.001$ & .914 & .894 & .135 & .093 & .071 & .114 \\
$\begin{array}{l}\text { factors } \\
13 \text { items, }\end{array}$ & 119.382 & 64 & 1.865 & $<.001$ & .933 & .919 & .048 & .082 & .059 & .104 \\
$\quad$ corr \\
factors
\end{tabular}




\section{APPENDIX K}

\section{HUMAN SUBJECTS APPROVAL}

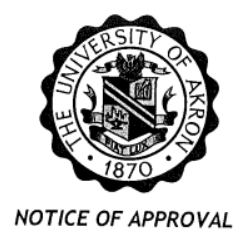

Date: June 23, 2009

To: $\quad$ Erin MacDougall

2101 Ridgebury Drive

Painesville, Ohio 44077

From: Sharon McWhorter, IRB Administrator

Re: IRB Number 20090610 "An Examination of a Model of Intuitive Eating with AfricanAmerican College Women"

Thank you for submitting your Exemption Request for the referenced study. Your request was approved on June 23,2009 . The protocol represents minimal risk to subjects and matches the following federal

$\square$ Exemption 1 - Research conducted in established or commonly accepted educational settings, involving normal educational practices.

$\triangle$ Exemption 2 - Research involving the use of educational tests, survey procedures, interview procedures, or observation of public behavior.

$\square$ Exemption 3 - Research involving the use of educational tests, survey procedures, interview procedures, or observation of public behavior not exempt under category 2, but subjects are elected or appointed public officials or candidates for public office.

$\square$ Exemption 4 - Research involving the collection or study of existing data, documents, records, pathological specimens, or diagnostic specimens.

$\square$ Exemption 5 - Research and demonstration projects conducted by or subject to the approval of department or agency heads, and which are designed to study, evaluate, or otherwise examine public programs or benefits.

$\square$ Exemption 6 - Taste and food quality evaluation and consumer acceptance studies.

Annual continuation applications are not required for exempt projects. If you make changes to the study's design or procedures that increase the risk to subjects or include activities that do not fall within the approved exemption category, please contact me to discuss whether or not a new application must be submitted. Any such changes or modifications must be reviewed and approved by

Please retain this letter for your files. If the research is being conducted for a master's thesis or doctoral dissertation, the student must file a copy of this letter with the thesis or dissertation.

Cc: Linda Subich - Advisor

\Approved consent form/s enclosed

Cc: Stephanie Woods - IRB Chair

Office of Research Services and Sponsored Programs

Akron, $\mathrm{OH} 44325-2102$

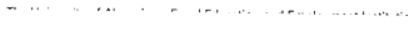

